\title{
Thymol as an interesting building block for promising fungicides against Fusarium solani
}

\author{
Mariana Alves Eloy, ${ }^{a}$ Rayssa Ribeiro, ${ }^{a}$ Leandra Martins Meireles, ${ }^{b}$ Thiago Antonio \\ de Sousa Cutrim, ${ }^{b}$ Carla Santana Francisco, ${ }^{c}$ Clara Lirian Javarini, ${ }^{c}$ Warley de \\ Souza Borges, ${ }^{c}$ Adilson Vidal Costa, ${ }^{a}$ Vagner Tebaldi de Queiroz, ${ }^{a}$ Rodrigo Scherer, ${ }^{b}$ \\ Valdemar Lacerda Jr., *\#c Pedro Alves Bezerra Morais**a
}

aPrograma de Pós-Graduação em Agroquímica, Universidade Federal do Espírito

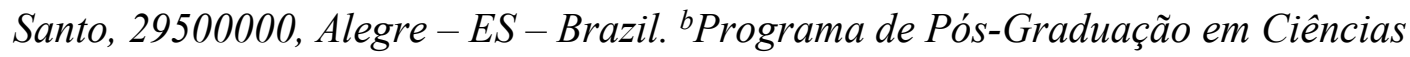
Farmacêuticas, Universidade Vila Velha, 29103-900, Vila Velha-ES-Brazil. cPrograma de Pós-Graduação em Química, Universidade Federal do Espírito Santo, 29075910, Vitória-ES-Brazil.

\footnotetext{
\# Both authors contributed equally to this work *pedro.morais@ufes.br; vljuniorqui@gmail.com Orcid P.A.B.M.: 0000-0001-5501-7350
} 


\section{Supporting Information}

Figure S1. Compound 2a and main $\mathrm{HMBC}$ correlation $J$ in $\mathrm{Hz}$.

Table S1. Spectroscopic data for compound 2a.

Figure S2. ${ }^{1} \mathrm{H}$ NMR spectrum $\left(400 \mathrm{MHz}, \mathrm{CDCl}_{3}\right.$ ) of compound 1-isopropyl-4-methyl-2-(prop-2yn-1-yloxy)benzene (2a).

Figure S3. ${ }^{13} \mathrm{C}$ NMR spectrum $\left(100 \mathrm{MHz}, \mathrm{CDCl}_{3}\right)$ of compound 1-isopropyl-4-methyl-2-(prop2-yn-1-yloxy)benzene (2a).

Figure $\mathbf{S 4}$ - $\mathrm{HMBC}$ spectrum ( $400 \mathrm{MHz}, \mathrm{CDCl}_{3}$ ) of the compound 1-isopropyl-4-methyl-2-(prop2-yn-1-yloxy)benzene (2a).

Figure S5. IR spectrum of compound $\mathbf{2 a}$.

Figure S6. HRMS spectrum of compound 2a.

Figure S7. Compound $\mathbf{2 b}$ and main HMBC correlation $J$ in $\mathrm{Hz}$.

Table S2. Spectroscopic data for compound $\mathbf{2 b}$.

Figure S8. ${ }^{1} \mathrm{H}$ NMR spectrum $\left(400 \mathrm{MHz}, \mathrm{CDCl}_{3}\right.$ ) of compound (2-chloro-3-(2-isopropyl-5methylphenoxy)naphthalene-1,4-dione) (2b).

Figure S9. ${ }^{13} \mathrm{C}$ NMR spectrum $\left(100 \mathrm{MHz}, \mathrm{CDCl}_{3}\right)$ of compound (2-chloro-3-(2-isopropyl-5methylphenoxy)naphthalene-1,4-dione) (2b).

Figure S10 - HMBC spectrum (400 MHz, $\mathrm{CDCl}_{3}$ ) of the compound (2-chloro-3-(2-isopropyl-5methylphenoxy)naphthalene-1,4-dione) (2b).

Figure S11 - HSQC spectrum (400 MHz, $\mathrm{CDCl}_{3}$ ) of the compound (2-chloro-3-(2-isopropyl-5methylphenoxy)naphthalene-1,4-dione) (2b).

Figure S12. IR spectrum of compound $\mathbf{2 b}$.

Figure S13. HRMS spectrum of compound $\mathbf{2 b}$.

Figure S14. Compound 2c and main $\mathrm{HMBC} J$ in $\mathrm{Hz}$.

Table S3. Spectroscopic data for compound 2c.

Figure S15. ${ }^{1} \mathrm{H}$ NMR spectrum $\left(400 \mathrm{MHz}, \mathrm{CDCl}_{3}\right)$ of compound (2-((2-isopropyl-5methylphenoxy)methyl)-1H-indene-1,3(2H)-dione) (2c).

Figure S16. ${ }^{13} \mathrm{C}$ NMR spectrum $\left(100 \mathrm{MHz}, \mathrm{CDCl}_{3}\right)$ of compound (2-((2-isopropyl-5methylphenoxy)methyl)-1 $H$-indene-1,3(2H)-dione) (2c).

Figure S17 - HMBC spectrum $\left(400 \mathrm{MHz}, \mathrm{CDCl}_{3}\right)$ of the compound (2-((2-isopropyl-5methylphenoxy)methyl)-1H-indene-1,3(2H)-dione) (2c).

Figure S18. HSQC spectrum $\left(400 \mathrm{MHz}, \mathrm{CDCl}_{3}\right)$ of the compound (2-((2-isopropyl-5methylphenoxy)methyl)-1H-indene-1,3(2H)-dione) (2c).

Figure S19. IR spectrum of compound 2c.

Figure S20. HRMS spectrum of compound 2c.

Figure S21. Compound 2d and main $\mathrm{HMBC} J$ in $\mathrm{Hz}$. 
Table S4. Spectroscopic data for compound $\mathbf{2 d}$.

Figure S22. ${ }^{1} \mathrm{H}$ NMR spectrum (400 $\mathrm{MHz}, \mathrm{CDCl}_{3}$ ) of compound (1-isopropyl-4-methyl-2-((4nitrobenzyl)oxy)benzene) (2d).

Figure S23. ${ }^{13} \mathrm{C}$ NMR spectrum $\left(100 \mathrm{MHz}, \mathrm{CDCl}_{3}\right)$ of compound (1-isopropyl-4-methyl-2-((4nitrobenzyl)oxy)benzene) (2d).

Figure S24 - HMBC spectrum (400 MHz, $\left.\mathrm{CDCl}_{3}\right)$ of the compound (1-isopropyl-4-methyl-2-((4nitrobenzyl)oxy)benzene) (2d).

Figure S25. IR spectrum of compound 2d.

Figure S26. HRMS spectrum of compound 2d.

Figure S27. Compound 2e and main HMBC correlation $J$ in $\mathrm{Hz}$.

Table S5. Spectroscopic data for compound 2e.

Figure S28. ${ }^{1} \mathrm{H}$ NMR spectrum (400 $\mathrm{MHz}, \mathrm{CDCl}_{3}$ ) of compound (2-(2-isopropyl-5methylphenoxy)acetonitrile) (2e).

Figure S29. ${ }^{13} \mathrm{C}$ NMR spectrum $\left(100 \mathrm{MHz}, \mathrm{CDCl}_{3}\right)$ of compound (2-(2-isopropyl-5methylphenoxy)acetonitrile) (2e).

Figure S30 - $\mathrm{HMBC}$ spectrum $\left(400 \mathrm{MHz}, \mathrm{CDCl}_{3}\right)$ of the compound (2-(2-isopropyl-5methylphenoxy)acetonitrile) (2e).

Figure S31. IR spectrum of compound 2e.

Figure S32. HRMS spectrum of compound 2e.

Figure S33. Compound $2 \mathrm{f}$ and main $\mathrm{HMBC}$ correlation $J$ in $\mathrm{Hz}$.

Table S6. Spectroscopic data for compound $2 \mathrm{f}$.

Figure S34. ${ }^{1} \mathrm{H}$ NMR spectrum $\left(400 \mathrm{MHz}, \mathrm{CDCl}_{3}\right)$ of compound (1-isopropyl-4-methyl-2-((2((phenylsulfonyl)methyl)benzyl)oxy)benzene) (2f).

Figure S35. ${ }^{13} \mathrm{C}$ NMR spectrum $\left(100 \mathrm{MHz}, \mathrm{CDCl}_{3}\right)$ of compound (1-isopropyl-4-methyl-2-((2((phenylsulfonyl)methyl)benzyl)oxy)benzene) (2f).

Figure $\mathbf{S 3 6}$ - $\mathrm{HMBC}$ spectrum $\left(400 \mathrm{MHz}, \mathrm{CDCl}_{3}\right)$ of the compound (1-isopropyl-4-methyl-2-((2((phenylsulfonyl)methyl)benzyl)oxy)benzene) (2f).

Figure S37. IR spectrum of compound $2 f$.

Figure S38. HRMS spectrum of compound $2 \mathbf{f}$.

Figure S39. Compound 3d and main $\mathrm{HMBC}$ correlation $J$ in $\mathrm{Hz}$.

Table S7. Spectroscopic data for compound 3d.

Figure S40. ${ }^{1} \mathrm{H}$ NMR spectrum $\left(400 \mathrm{MHz}, \mathrm{CDCl}_{3}\right)$ of compound (1-(3-bromophenyl)-4-((2isopropyl-5-methylphenoxy)methyl)-1 $H$-1,2,3-triazole) (3d).

Figure S41. ${ }^{13} \mathrm{C}$ NMR spectrum $\left(100 \mathrm{MHz}, \mathrm{CDCl}_{3}\right)$ of compound (1-(3-bromophenyl)-4-((2isopropyl-5-methylphenoxy)methyl)-1 $H$-1,2,3-triazole) (3d).

Figure S42 - HMBC spectrum (400 MHz, $\mathrm{CDCl}_{3}$ ) of the compound (1-(3-bromophenyl)-4-((2isopropyl-5-methylphenoxy)methyl)-1 $H$-1,2,3-triazole) (3d). 
Figure S43 - HSQC spectrum (400 $\left.\mathrm{MHz}, \mathrm{CDCl}_{3}\right)$ of the compound (1-(3-bromophenyl)-4-((2isopropyl-5-methylphenoxy)methyl)-1 $H$-1,2,3-triazole) (3d).

Figure S44. IR spectrum of compound 3d.

Figure S45. HRMS spectrum of compound 3d.

Figure S46. Compound 3e and main $\mathrm{HMBC}$ correlation $J$ in $\mathrm{Hz}$

Table S8. Spectroscopic data for compound 3e.

Figure S47. ${ }^{1} \mathrm{H}$ NMR spectrum (400 $\left.\mathrm{MHz}, \mathrm{CDCl}_{3}\right)$ of compound (4-((2-isopropyl-5methylphenoxy)methyl)-1-(3-(trifluoromethyl)phenyl)-1 $H$-1,2,3-triazole) (3e).

Figure S48. ${ }^{13} \mathrm{C}$ NMR spectrum $\left(100 \mathrm{MHz}, \mathrm{CDCl}_{3}\right)$ of compound (4-((2-isopropyl-5methylphenoxy)methyl)-1-(3-(trifluoromethyl)phenyl)-1 $H$-1,2,3-triazole) (3e).

Figure S49 - $\mathrm{HMBC}$ spectrum $\left(400 \mathrm{MHz}, \mathrm{CDCl}_{3}\right)$ of the compound (4-((2-isopropyl-5methylphenoxy)methyl)-1-(3-(trifluoromethyl)phenyl)-1H-1,2,3-triazole) (3e).

Figure S50 - HSQC spectrum (400 $\left.\mathrm{MHz}, \mathrm{CDCl}_{3}\right)$ of the compound (4-((2-isopropyl-5methylphenoxy)methyl)-1-(3-(trifluoromethyl)phenyl)-1 $H$-1,2,3-triazole) (3e).

Figure S51. IR spectrum of compound 3e.

Figure S52. HRMS spectrum of compound 3e.

Figure S53. Compound $\mathbf{3 f}$ and main $\mathrm{HMBC}$ correlation $J$ in $\mathrm{Hz}$.

Table S9. Spectroscopic data for compound 3f.

Figure S54. ${ }^{1} \mathrm{H}$ NMR spectrum $\left(400 \mathrm{MHz}, \mathrm{CDCl}_{3}\right)$ of compound (1-(2-bromophenyl)-4-((2isopropyl-5-methylphenoxy)methyl)-1 $H$-1,2,3-triazole) (3f).

Figure S55. ${ }^{13} \mathrm{C}$ NMR spectrum $\left(100 \mathrm{MHz}, \mathrm{CDCl}_{3}\right)$ of compound (1-(2-bromophenyl)-4-((2isopropyl-5-methylphenoxy)methyl)-1 $H$-1,2,3-triazole) (3f).

Figure S56 - $\mathrm{HMBC}$ spectrum $\left(400 \mathrm{MHz}, \mathrm{CDCl}_{3}\right)$ of the compound (1-(2-bromophenyl)-4-((2isopropyl-5-methylphenoxy)methyl)-1 $H$-1,2,3-triazole) (3f).

Figure S57 - HSQC spectrum (400 MHz, $\left.\mathrm{CDCl}_{3}\right)$ of the compound (1-(2-bromophenyl)-4-((2isopropyl-5-methylphenoxy)methyl)-1 $H$-1,2,3-triazole) (3f).

Figure S58. IR spectrum of compound $3 f$.

Figure S59. HRMS spectrum of compound $3 \mathbf{f}$.

Figure S60. Compound $\mathbf{3 g}$ and main $\mathrm{HMBC}$ correlation $J$ in $\mathrm{Hz}$.

Table S10. Spectroscopic data for compound 3g.

Figure S61. ${ }^{1} \mathrm{H}$ NMR spectrum (400 $\left.\mathrm{MHz}, \mathrm{CDCl}_{3}\right)$ of compound (4-((2-isopropyl-5methylphenoxy)methyl)-1-(2-methoxyphenyl)-1H-1,2,3-triazole) (3g).

Figure S62. ${ }^{13} \mathrm{C}$ NMR spectrum $\left(100 \mathrm{MHz}, \mathrm{CDCl}_{3}\right)$ of compound (4-((2-isopropyl-5methylphenoxy)methyl)-1-(2-methoxyphenyl)-1H-1,2,3-triazole) (3g).

Figure S63 - HMBC spectrum $\left(400 \mathrm{MHz}, \mathrm{CDCl}_{3}\right)$ of the compound (4-((2-isopropyl-5methylphenoxy)methyl)-1-(2-methoxyphenyl)-1H-1,2,3-triazole) (3g).

Figure S64 - HSQC spectrum $\left(400 \mathrm{MHz}, \mathrm{CDCl}_{3}\right)$ of the compound (4-((2-isopropyl-5methylphenoxy)methyl)-1-(2-methoxyphenyl)-1H-1,2,3-triazole) (3g). 
Figure S65. IR spectrum of compound 3g.

Figure S66. HRMS spectrum of compound 3g.

Figure S67. Compound $\mathbf{3 h}$ and main $\mathrm{HMBC}$ correlation $\mathrm{J}$ in $\mathrm{Hz}$.

Table S11. Spectroscopic data for compound $\mathbf{3 h}$.

Figure S68. ${ }^{1} \mathrm{H}$ NMR spectrum (400 $\left.\mathrm{MHz}, \mathrm{CDCl}_{3}\right)$ of compound (4-((2-isopropyl-5methylphenoxy)methyl)-1-(4-methoxyphenyl)-1H-1,2,3-triazole) (3h).

Figure S69. ${ }^{13} \mathrm{C}$ NMR spectrum $\left(100 \mathrm{MHz}, \mathrm{CDCl}_{3}\right)$ of compound (4-((2-isopropyl-5methylphenoxy)methyl)-1-(4-methoxyphenyl)-1H-1,2,3-triazole) (3h).

Figure S70 - HMBC spectrum $\left(400 \mathrm{MHz}, \mathrm{CDCl}_{3}\right)$ of the compound (4-((2-isopropyl-5methylphenoxy)methyl)-1-(4-methoxyphenyl)-1H-1,2,3-triazole) (3h).

Figure S71 - HSQC spectrum (400 $\left.\mathrm{MHz}, \mathrm{CDCl}_{3}\right)$ of the compound (4-((2-isopropyl-5methylphenoxy)methyl)-1-(4-methoxyphenyl)-1H-1,2,3-triazole) (3h).

Figure S72. IR spectrum of compound $3 \mathbf{h}$.

Figure S73. HRMS spectrum of compound $\mathbf{3 h}$.

Figure S74. Compound $\mathbf{3 i}$ and main $\mathrm{HMBC}$ correlation $J$ in $\mathrm{Hz}$.

Table S12. Spectroscopic data for compound $3 \mathbf{i}$.

Figure S75. ${ }^{1} \mathrm{H}$ NMR spectrum $\left(400 \mathrm{MHz}, \mathrm{CDCl}_{3}\right)$ of compound (4-((2-isopropyl-5methylphenoxy)methyl)-1-(3-methoxyphenyl)-1H-1,2,3-triazole) (3i).

Figure S76. ${ }^{13} \mathrm{C}$ NMR spectrum $\left(100 \mathrm{MHz}, \mathrm{CDCl}_{3}\right)$ of compound (4-((2-isopropyl-5methylphenoxy)methyl)-1-(3-methoxyphenyl)-1H-1,2,3-triazole) (3i).

Figure S77 - HMBC spectrum $\left(400 \mathrm{MHz}, \mathrm{CDCl}_{3}\right)$ of the compound (4-((2-isopropyl-5methylphenoxy)methyl)-1-(3-methoxyphenyl)-1H-1,2,3-triazole) (3i).

Figure S78 - HSQC spectrum (400 $\left.\mathrm{MHz}, \mathrm{CDCl}_{3}\right)$ of the compound (4-((2-isopropyl-5methylphenoxy)methyl)-1-(3-methoxyphenyl)-1H-1,2,3-triazole) (3i).

Figure S79. IR spectrum of compound 3i.

Figure S80. HRMS spectrum of compound 3i.

Figure S81. Compound $\mathbf{3 j}$ and main $\mathrm{HMBC}$ correlation $J$ in $\mathrm{Hz}$.

Table S13. Spectroscopic data for compound $\mathbf{3 j}$.

Figure S82. ${ }^{1} \mathrm{H}$ NMR spectrum $\left(400 \mathrm{MHz}, \mathrm{CDCl}_{3}\right)$ of compound (4-((4-)((2-isopropyl-5methylphenoxy)methyl)-1H-1,2,3-triazol-1-yl)methyl)benzoic acid) (3j).

Figure S83. ${ }^{13} \mathrm{C}$ NMR spectrum $\left(100 \mathrm{MHz}, \mathrm{CDCl}_{3}\right)$ of compound (4-((4-((2-isopropyl-5methylphenoxy)methyl)-1H-1,2,3-triazol-1-yl)methyl)benzoic acid) (3j).

Figure S84 - HMBC spectrum $\left(400 \mathrm{MHz}, \mathrm{CDCl}_{3}\right)$ of the compound (4-((4-((2-isopropyl-5methylphenoxy)methyl)-1H-1,2,3-triazol-1-yl)methyl)benzoic acid) (3j).

Figure S85 - HSQC spectrum $\left(400 \mathrm{MHz}, \mathrm{CDCl}_{3}\right)$ of the compound (4-((4-((2-isopropyl-5methylphenoxy)methyl)-1H-1,2,3-triazol-1-yl)methyl)benzoic acid) (3j).

Figure S86. IR spectrum of compound $\mathbf{3 j}$. 
Figure S87. HRMS spectrum of compound 3j.

Figure S88. Compound 3k and main HMBC correlation $J$ in $\mathrm{Hz}$.

Table S14. Spectroscopic data for compound 3k.

Figure S89. ${ }^{1} \mathrm{H}$ NMR spectrum $\left(400 \mathrm{MHz}, \mathrm{CDCl}_{3}\right)$ of compound (4-((2-isopropyl-5methylphenoxy)methyl)-1-(2-nitrobenzyl)-1 $H$-1,2,3-triazole) (3k).

Figure S90. ${ }^{13} \mathrm{C}$ NMR spectrum $\left(100 \mathrm{MHz}, \mathrm{CDCl}_{3}\right)$ of compound (4-((2-isopropyl-5methylphenoxy)methyl)-1-(2-nitrobenzyl)-1H-1,2,3-triazole) (3k).

Figure S91 - HMBC spectrum $\left(400 \mathrm{MHz}, \mathrm{CDCl}_{3}\right)$ of the compound (4-((2-isopropyl-5methylphenoxy)methyl)-1-(2-nitrobenzyl)-1 $H$-1,2,3-triazole) (3k).

Figure S92 - HSQC spectrum (400 $\left.\mathrm{MHz}, \mathrm{CDCl}_{3}\right)$ of the compound (4-((2-isopropyl-5methylphenoxy)methyl)-1-(2-nitrobenzyl)-1 $H$-1,2,3-triazole) (3k).

Figure S93. IR spectrum of compound 3k.

Figure S94. HRMS spectrum of compound 3k.

Figure S95. Compound $\mathbf{3 I}$ and main $\mathrm{HMBC}$ correlation $J$ in $\mathrm{Hz}$.

Table S15. Spectroscopic data for compound 31.

Figure S96. ${ }^{1} \mathrm{H}$ NMR spectrum $\left(400 \mathrm{MHz}, \mathrm{CDCl}_{3}\right)$ of compound (4-((2-isopropyl-5methylphenoxy)methyl)-1-(3-nitrobenzyl)-1 $H$-1,2,3-triazole) (31).

Figure S97. ${ }^{13} \mathrm{C}$ NMR spectrum $\left(100 \mathrm{MHz}, \mathrm{CDCl}_{3}\right)$ of compound (4-((2-isopropyl-5methylphenoxy)methyl)-1-(3-nitrobenzyl)-1 $H$-1,2,3-triazole) (31).

Figure S98 - $\mathrm{HMBC}$ spectrum $\left(400 \mathrm{MHz}, \mathrm{CDCl}_{3}\right)$ of the compound (4-((2-isopropyl-5methylphenoxy)methyl)-1-(3-nitrobenzyl)-1 $H$-1,2,3-triazole) (31).

Figure S99 - HSQC spectrum (400 $\left.\mathrm{MHz}, \mathrm{CDCl}_{3}\right)$ of the compound (4-((2-isopropyl-5methylphenoxy)methyl)-1-(3-nitrobenzyl)-1 $H$-1,2,3-triazole) (3I).

Figure S100. IR spectrum of compound 31 .

Figure S101. HRMS spectrum of compound 31 . 


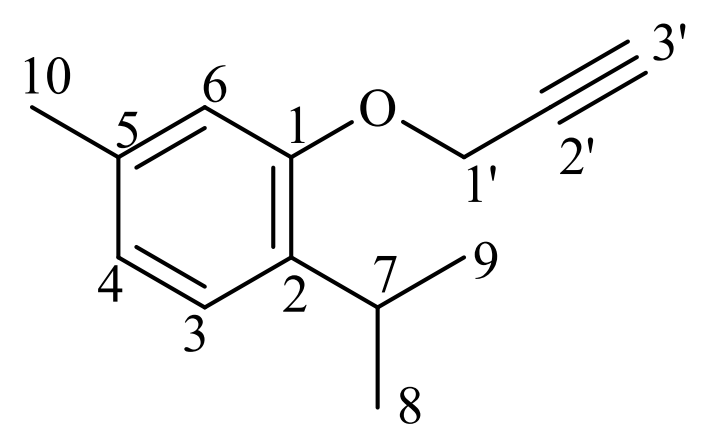

2a

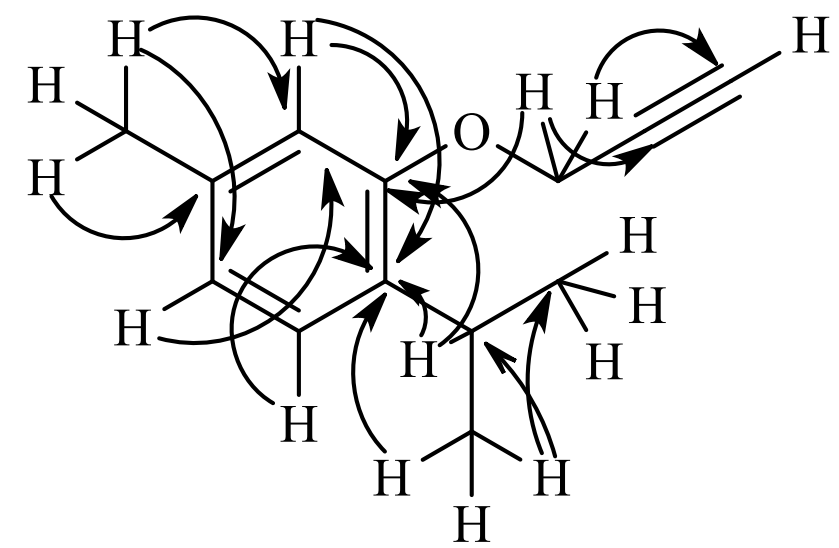

Figure S1. Compound 2a and main HMBC correlation $J$ in $\mathrm{Hz}$.
${ }^{1}$ H NMR (400 MHz, $1.20\left(6 \mathrm{H}, \mathrm{d}, \mathrm{CH}_{3}-8\right.$ and $\left.\mathrm{CH}_{3}-9\right) ; 2.32(3 \mathrm{H}, \mathrm{s}$, $\left.\left.\mathbf{C D C l}_{3}\right) \quad \mathrm{CH}_{3}-10\right) ; 2.48\left(1 \mathrm{H}, \mathrm{t}, J_{3},{ }_{1}, 2.44, \mathrm{H}-3\right.$ ') $3.26-3.34$ $(1 \mathrm{H}, \mathrm{m}, \mathrm{H}-7) ; 4.69\left(2 \mathrm{H}, \mathrm{d}, J_{1}, 3^{\prime}, 2.44, \mathrm{CH}_{2}-1^{\prime}\right)$ $6.76(1 \mathrm{H}, \mathrm{s}, \mathrm{H}-6) ; 6.79\left(1 \mathrm{H}, \mathrm{d}, J_{4,3} 7.82, \mathrm{H}-4\right)$; $7.11\left(1 \mathrm{H}, \mathrm{d}, J_{3,4} 7.82, \mathrm{H}-3\right)$

${ }^{13}$ C NMR (100 MHz, 21.33 (C-10); 22.86 (C-8, C-9); 26.45 (C-7) $\mathrm{CDCl}_{3}$ $56.11(\mathrm{C}-1$ ') $; 75.02(\mathrm{C}-3$ ') $; 79.14(\mathrm{C}-2$ ') $) ; 113.08$ (C-6); 122.23 (C-4); $126.12(\mathrm{C}-3) ; 134.74(\mathrm{C}-2)$; 136.26 (C-5); 154.75 (C-1).

Infra-red $\left(\mathrm{cm}^{-1}\right)$

3292 (C-H in C-3'); 2960 (C-H in $\mathrm{CH}_{2}-1^{\prime}, \mathrm{CH}-$ $7, \mathrm{CH}_{3}-8, \mathrm{CH}_{3}-9$ and $\left.\mathrm{CH}_{3}-10\right) ; 2852(\mathrm{C}-\mathrm{H}$ in $\mathrm{C}-$ 7); $2119\left(\mathrm{C} \equiv \mathrm{C}\right.$ in $\mathrm{C}-2^{\prime}$ ' and $\left.\mathrm{C}-3{ }^{\prime}\right) ; 1246$ and 1029 (ether in $\mathrm{C}-1$ ' and $\mathrm{C}-1$ ).

Mass spectrometry $\quad$ HRESIMS $[\mathrm{M}+\mathrm{Na}]^{+}$Found: 211.1090. Calc. for $\mathrm{C}_{13} \mathrm{H}_{16} \mathrm{NaO}^{+}: 211.1093$.

Table S1. Spectroscopic data for compound $\mathbf{2 a}$ 

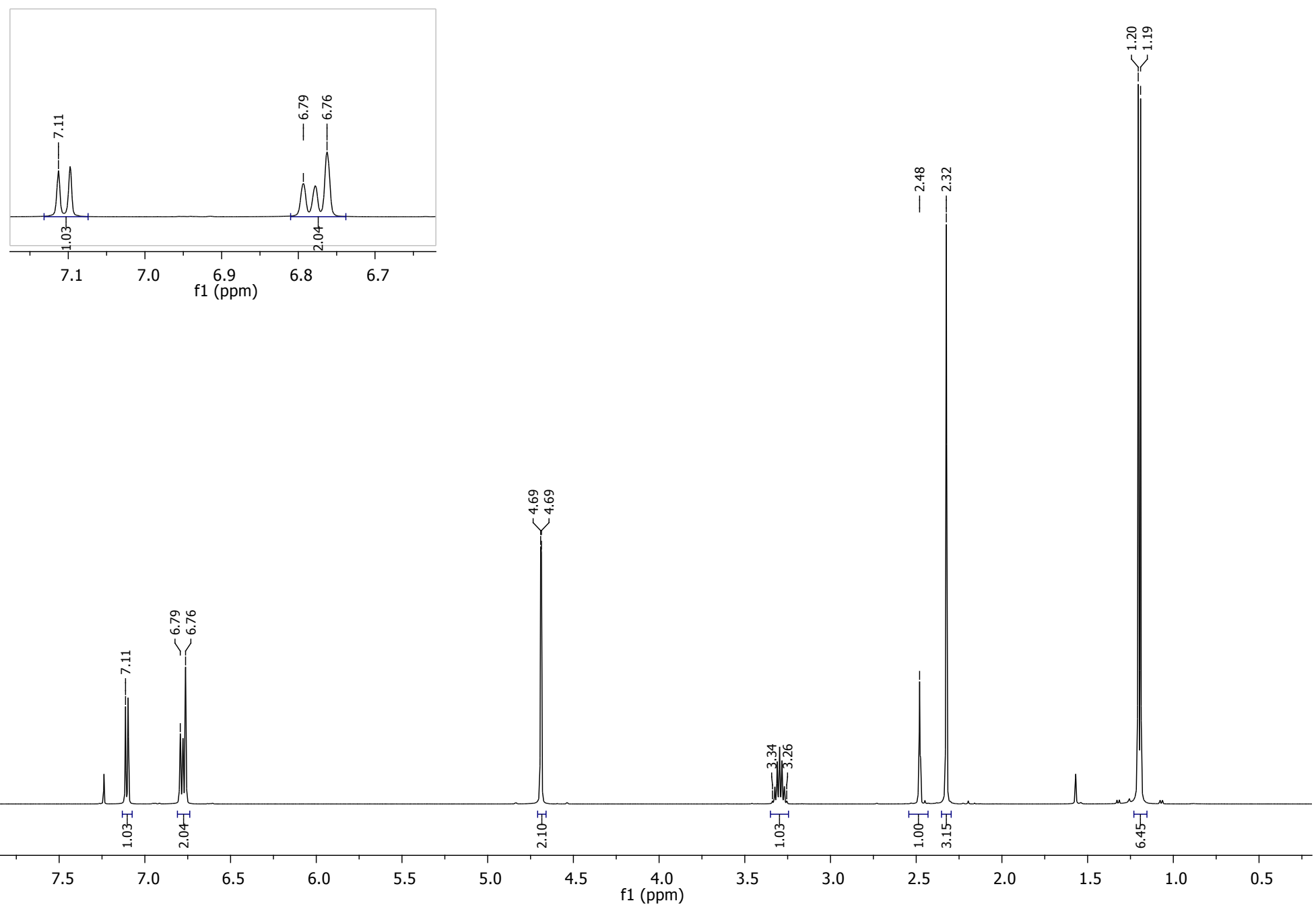

Figure S2. ${ }^{1} \mathrm{H}$ NMR spectrum (400 MHz, $\mathrm{CDCl}_{3}$ ) of compound 1-isopropyl-4-methyl-2-(prop-2-yn-1-yloxy)benzene (2a). 


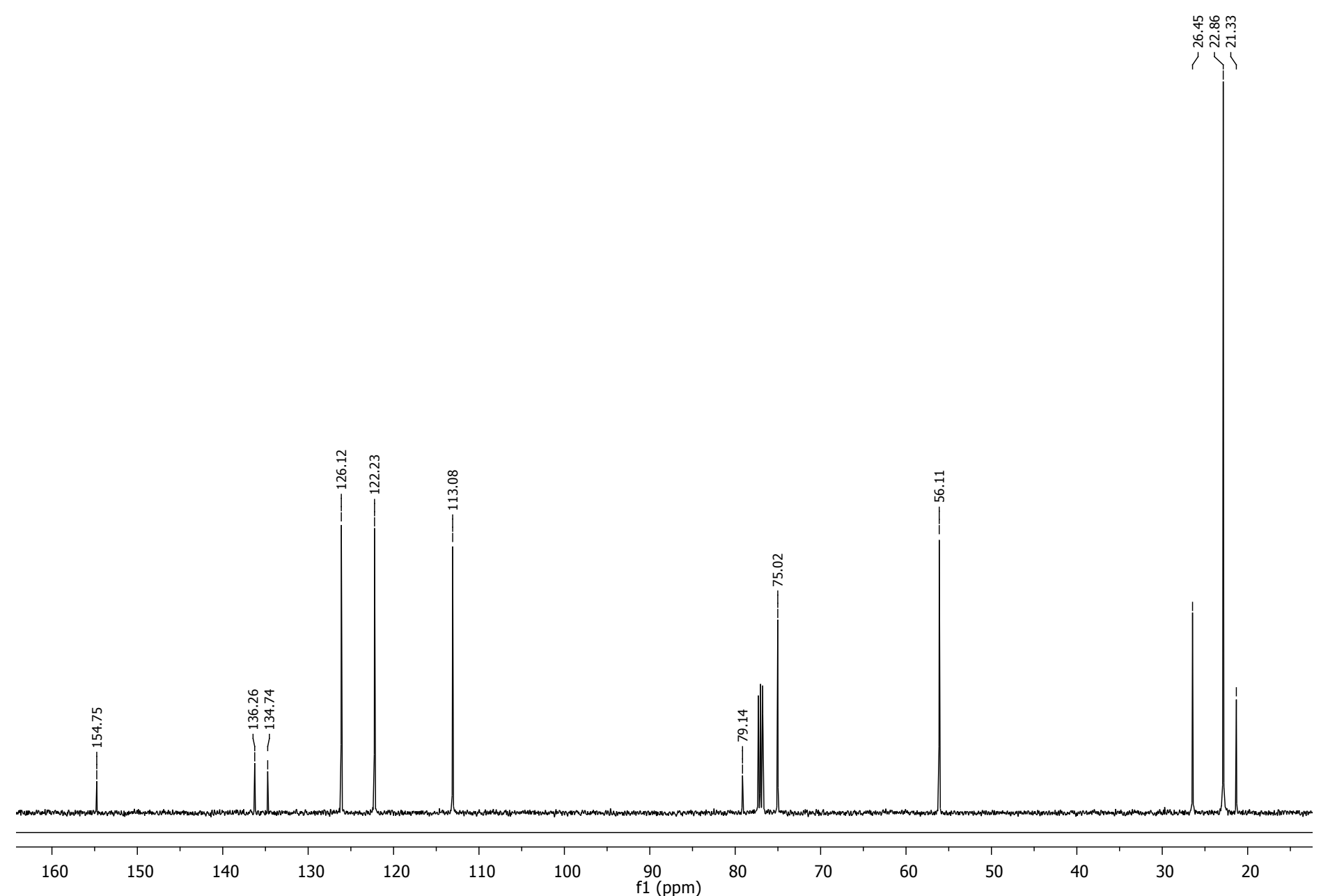

Figure S3. ${ }^{13} \mathrm{C}$ NMR spectrum $\left(100 \mathrm{MHz}, \mathrm{CDCl}_{3}\right.$ ) of compound 1-isopropyl-4-methyl-2-(prop-2-yn-1-yloxy)benzene (2a). 


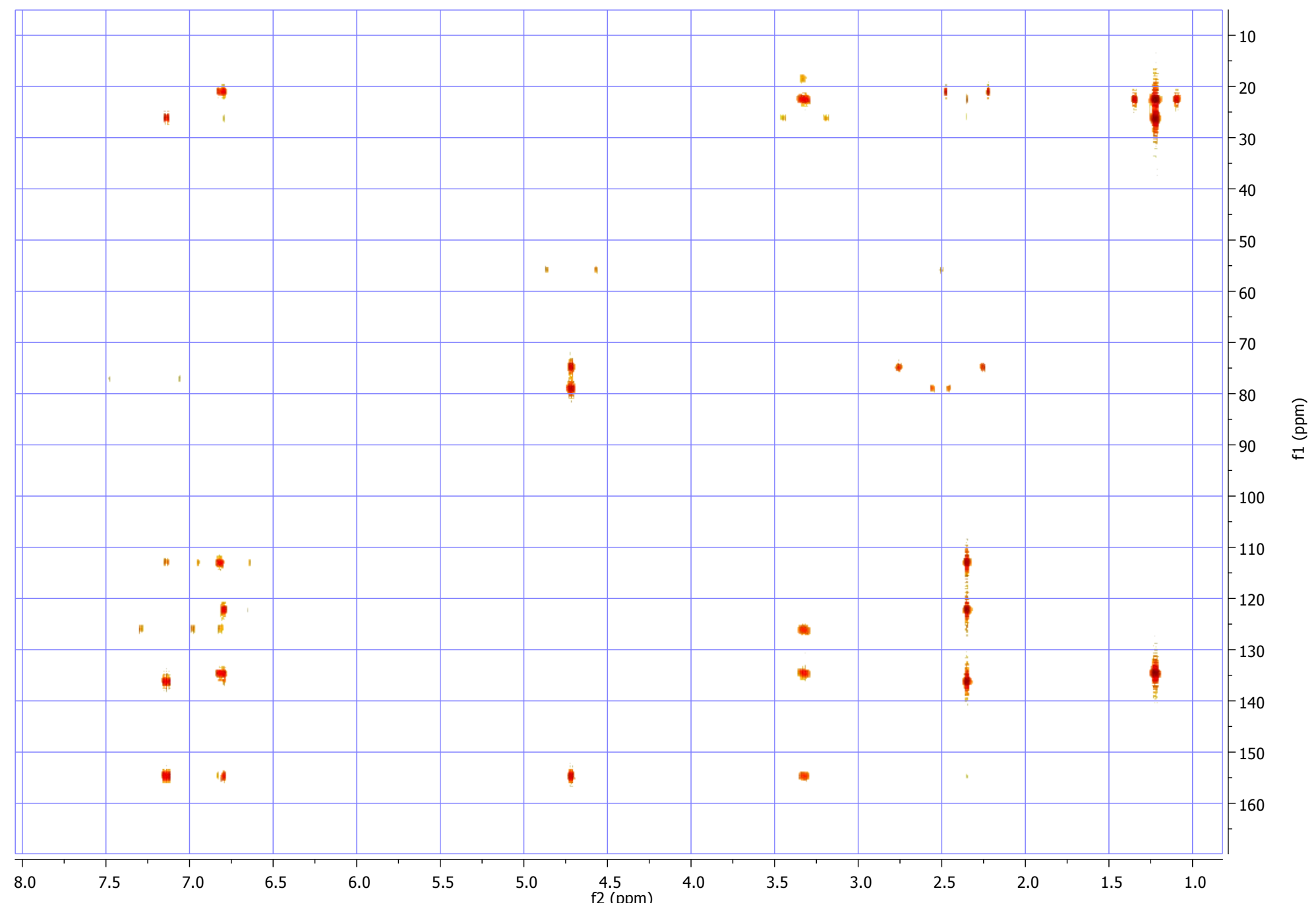

Figure S4 - $\mathrm{HMBC}$ spectrum (400 MHz, $\mathrm{CDCl}_{3}$ ) of the compound 1-isopropyl-4-methyl-2-(prop-2-yn-1-yloxy)benzene (2a). 


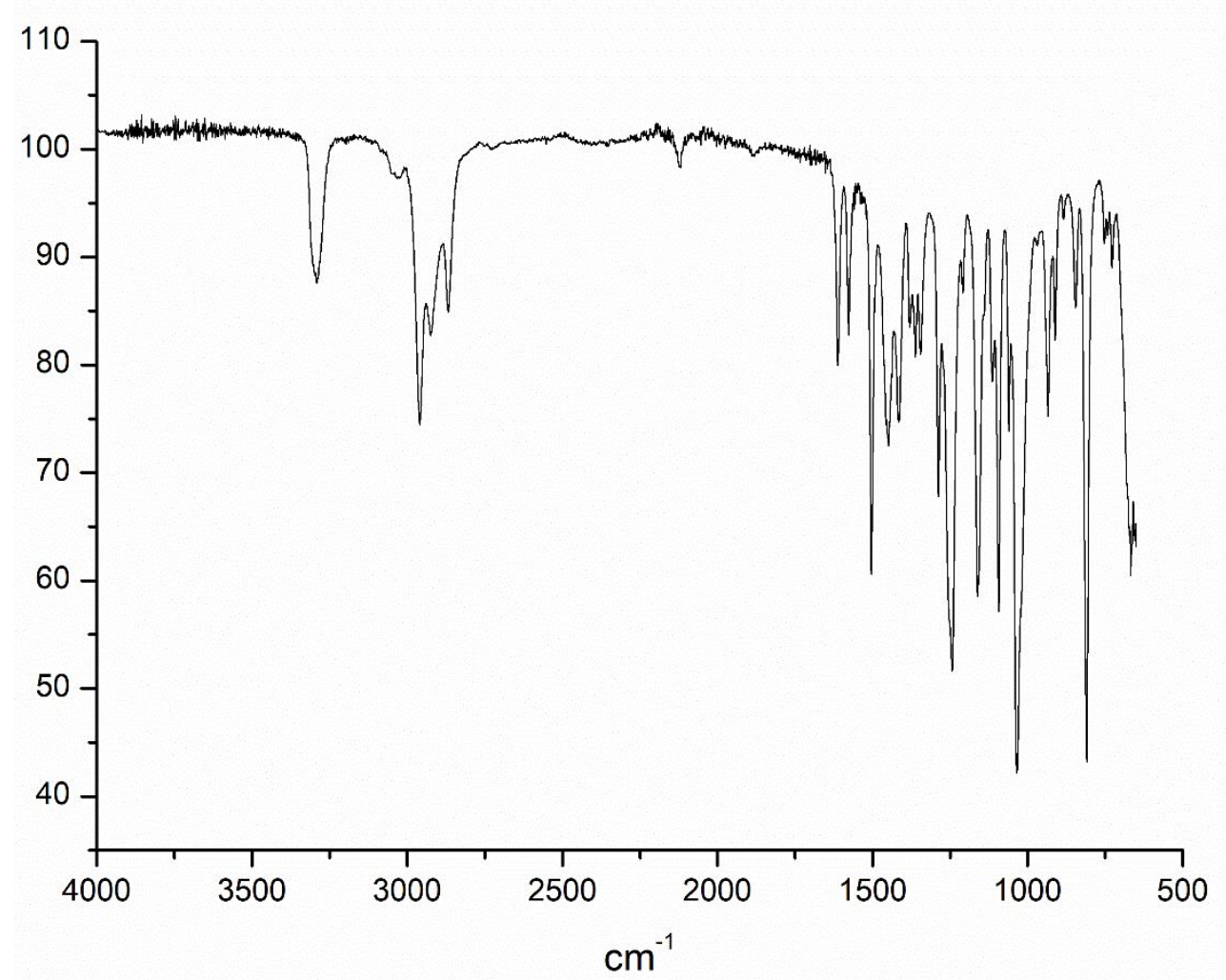

Figure S5. IR spectrum of compound 2a. 


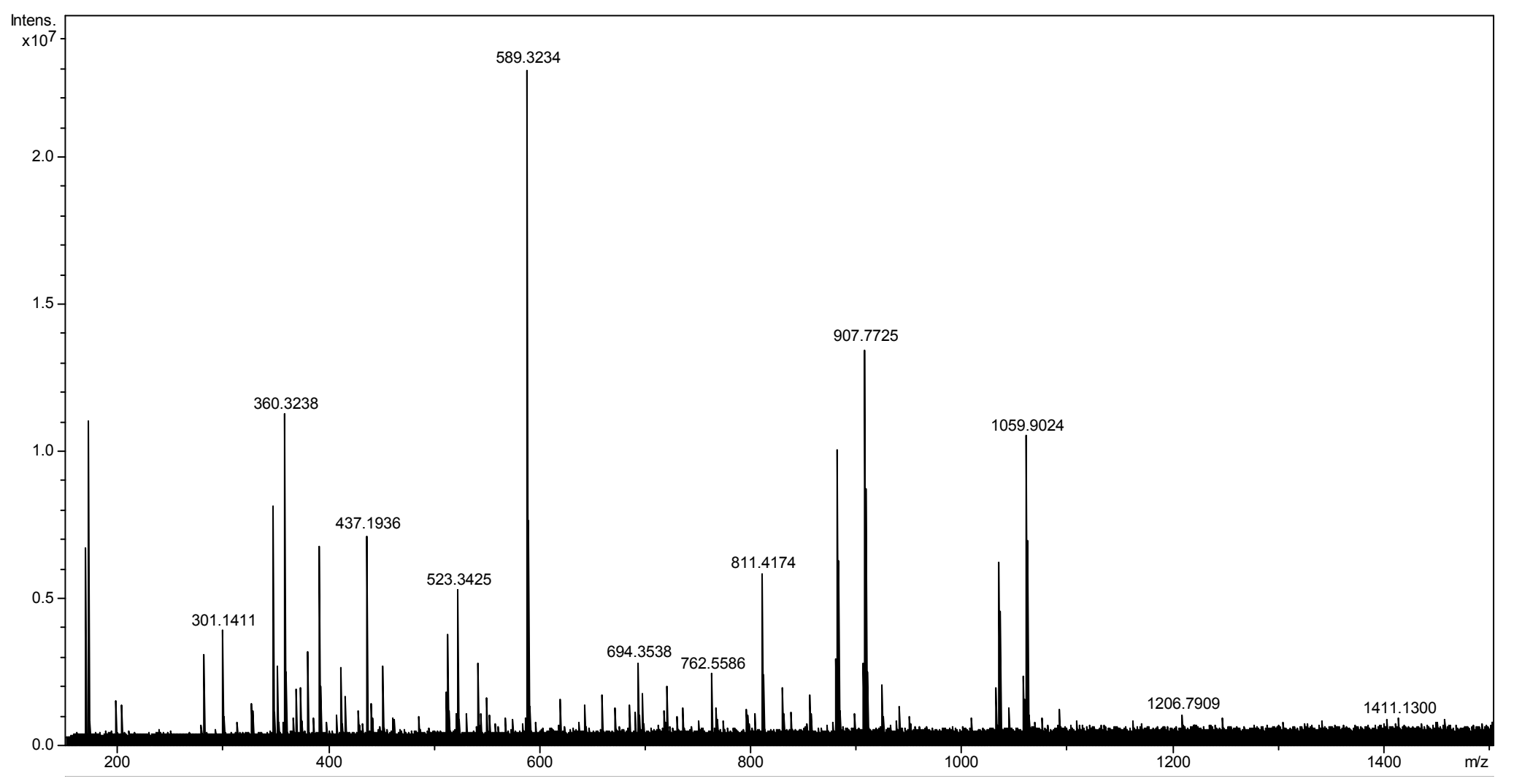

Figure S6. HRMS spectrum of compound $2 \mathbf{a}$. 
<smiles>CC(C)c1ccc(Br)cc1OC1=C(Cl)C(=O)c2ccccc2C1=O</smiles>

2b

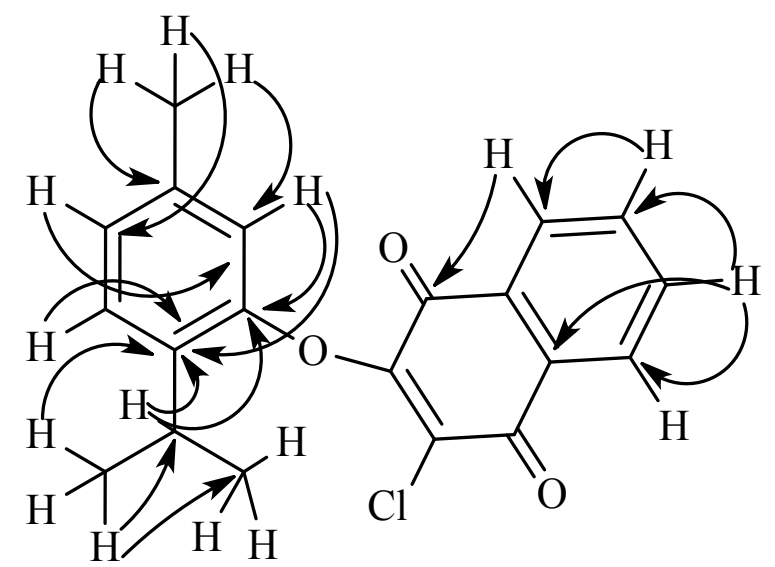

${ }^{1} \mathrm{H}$ NMR (400 MHz, $1.24\left(6 \mathrm{H}, \mathrm{d}, \mathrm{CH}_{3}-8\right.$ and $\left.\mathrm{CH}_{3}-9\right) ; 2.11(3 \mathrm{H}, \mathrm{s}$, DMSO- $d_{6}$ ) $\left.\mathrm{CH}_{3}-10\right) ; 3.28-3.33(1 \mathrm{H}, \mathrm{m}, \mathrm{H}-7) ; 6.80(1 \mathrm{H}, \mathrm{s}, \mathrm{H}-$ 6); $6.85\left(1 \mathrm{H}, \mathrm{d}, J_{4,3} 7.82, \mathrm{H}-4\right) ; 7.19\left(1 \mathrm{H}, \mathrm{d}, J_{3,4}\right.$ 7.82, H-3); $7.87\left(2 \mathrm{H}, \mathrm{m}, J_{4},{ }_{5}, 8.60, \mathrm{H}-4\right.$ ' and H$\left.5^{\prime}\right) ; 7.95\left(1 \mathrm{H}, \mathrm{dd}, J_{3},{ }_{6}, 8.60, \mathrm{H}-3\right.$ ') $8.09(1 \mathrm{H}, \mathrm{dd}$ $J_{6}, 4^{\prime}, 8.60$, H-6').

${ }^{13}$ C NMR (100 MHz, 20.75 (C-10); 22.99 (C-8, C-9); 27.00 (C-7); DMSO- $\left.d_{6}\right)$ 115.73 (C-6); 124.67 (C-4); $126.82 \quad(\mathrm{C}-3)$; 126.94 (C-3'); 127.01 (C-6'); 131.06 (C-2'); $131.89\left(\mathrm{C}^{\prime} 7^{\prime}\right) ; 133.85(\mathrm{C}-2) ; 134.80$ and 134.97 (C-4' and C-5'); 136.81 (C-5); 154.11 (C-1); 178.33 (C-1'); 178.54 (C-8').

Infra-red $\left(\mathrm{cm}^{-1}\right)$

2953 (C-H in $\mathrm{CH}-7, \mathrm{CH}_{3}-8, \mathrm{CH}_{3}-9, \mathrm{CH}_{3}-10$ ); $1663\left(\mathrm{C}=\mathrm{O}\right.$ in $\mathrm{C}-1^{\prime}$ ' and / or $\mathrm{C}-8$ ') 1237 and 1073 (ether in $\mathrm{C}-1$ and $\mathrm{C}-10$ '); 720 (C-Cl in C-9').

Mass spectrometry

HRESIMS [2M + Na] $]^{+}$Found: 703.1629. Calc. for $\mathrm{C}_{40} \mathrm{H}_{34} \mathrm{Cl}_{2} \mathrm{NaO}_{6}{ }^{+}$: 703.1625 .

Table S2. Spectroscopic data for compound $\mathbf{2 b}$.

Figure S7. Compound $\mathbf{2 b}$ and main $\mathrm{HMBC}$ correlation $J$ in $\mathrm{Hz}$. 

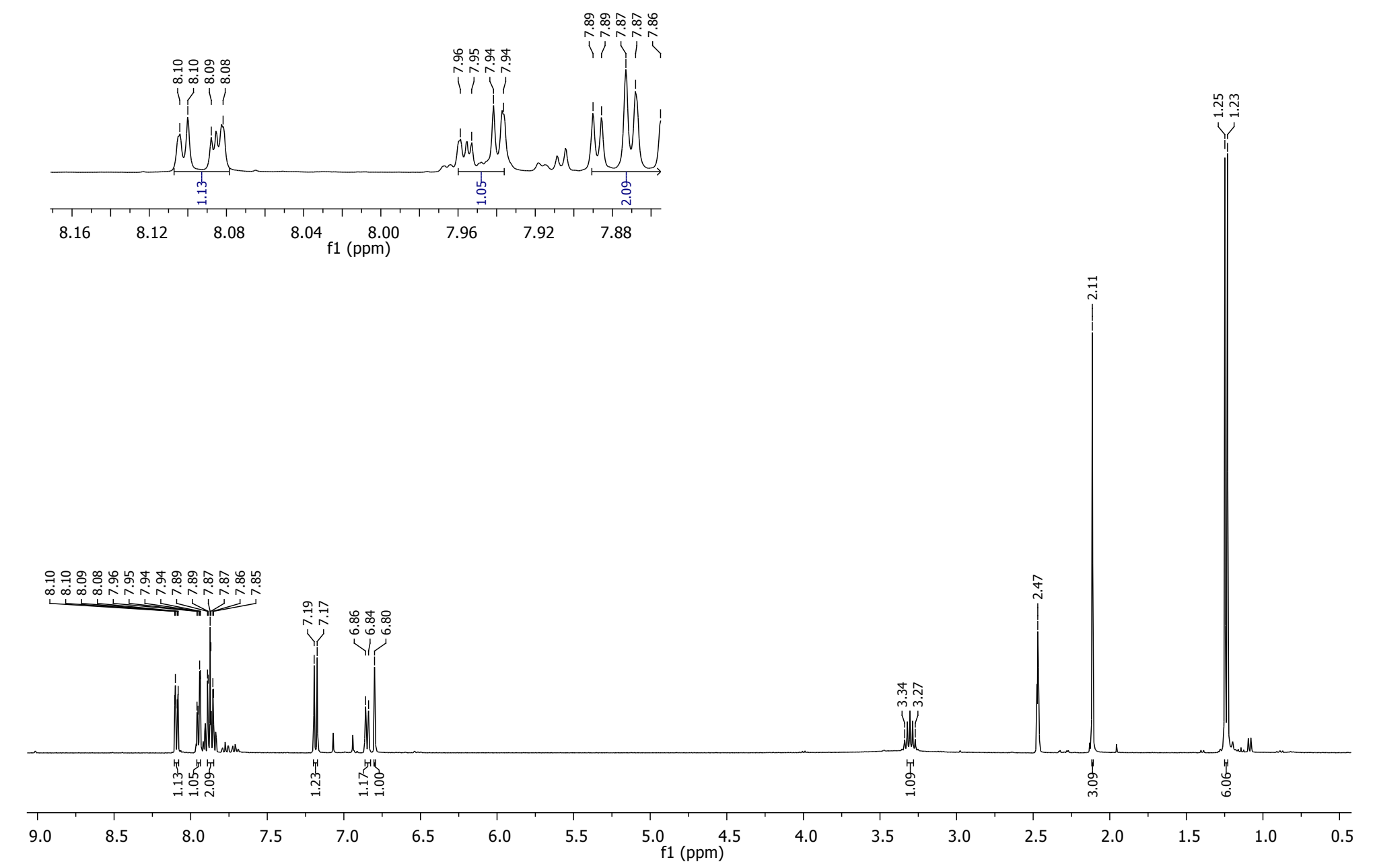

Figure S8. ${ }^{1} \mathrm{H}$ NMR spectrum (400 MHz, DMSO- $d_{6}$ ) of compound (2-chloro-3-(2-isopropyl-5-methylphenoxy)naphthalene-1,4-dione) (2b). 


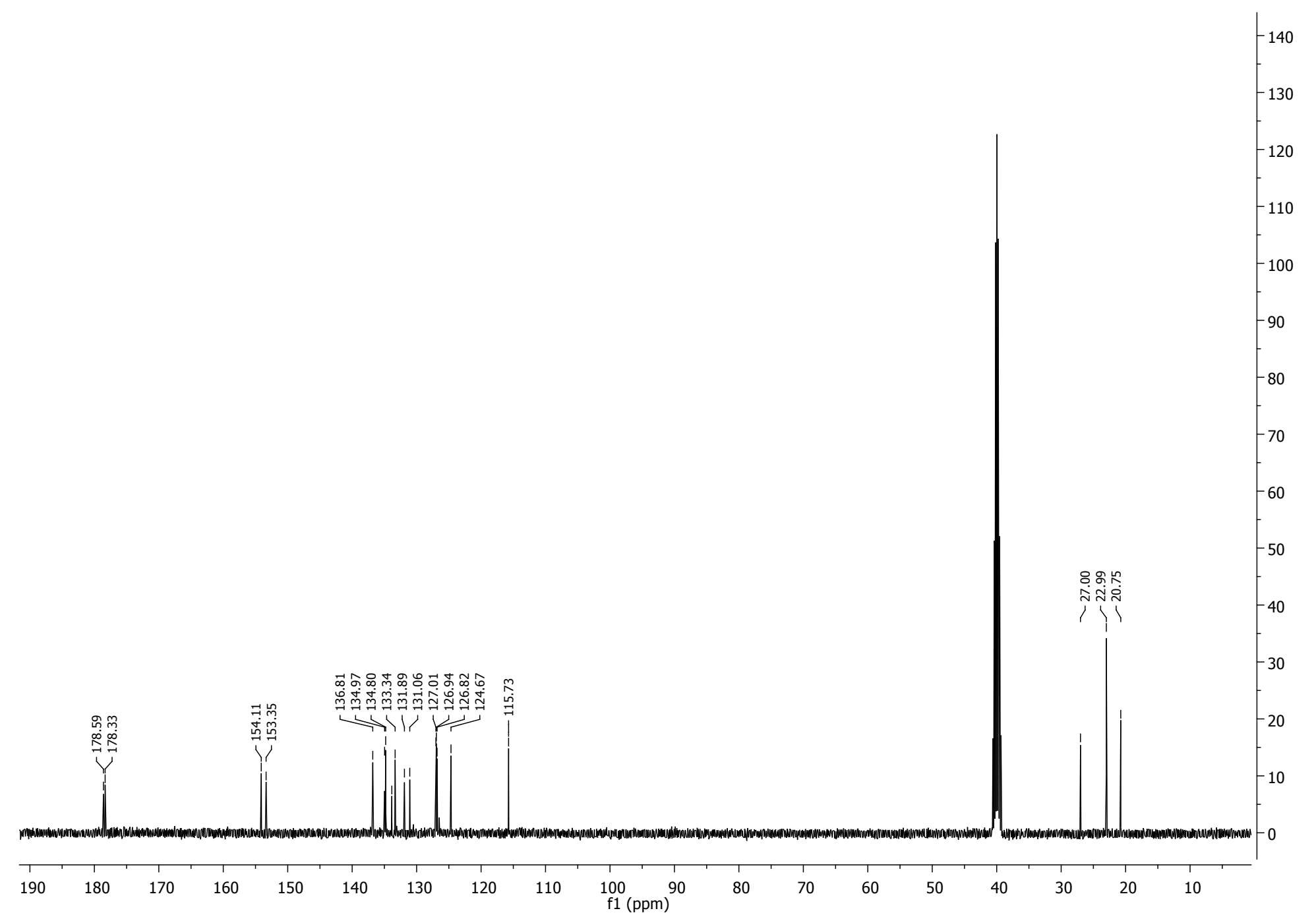

Figure S9. ${ }^{13} \mathrm{C}$ NMR spectrum (100 MHz, DMSO- $d_{6}$ ) of compound (2-chloro-3-(2-isopropyl-5-methylphenoxy)naphthalene-1,4-dione) (2b). 


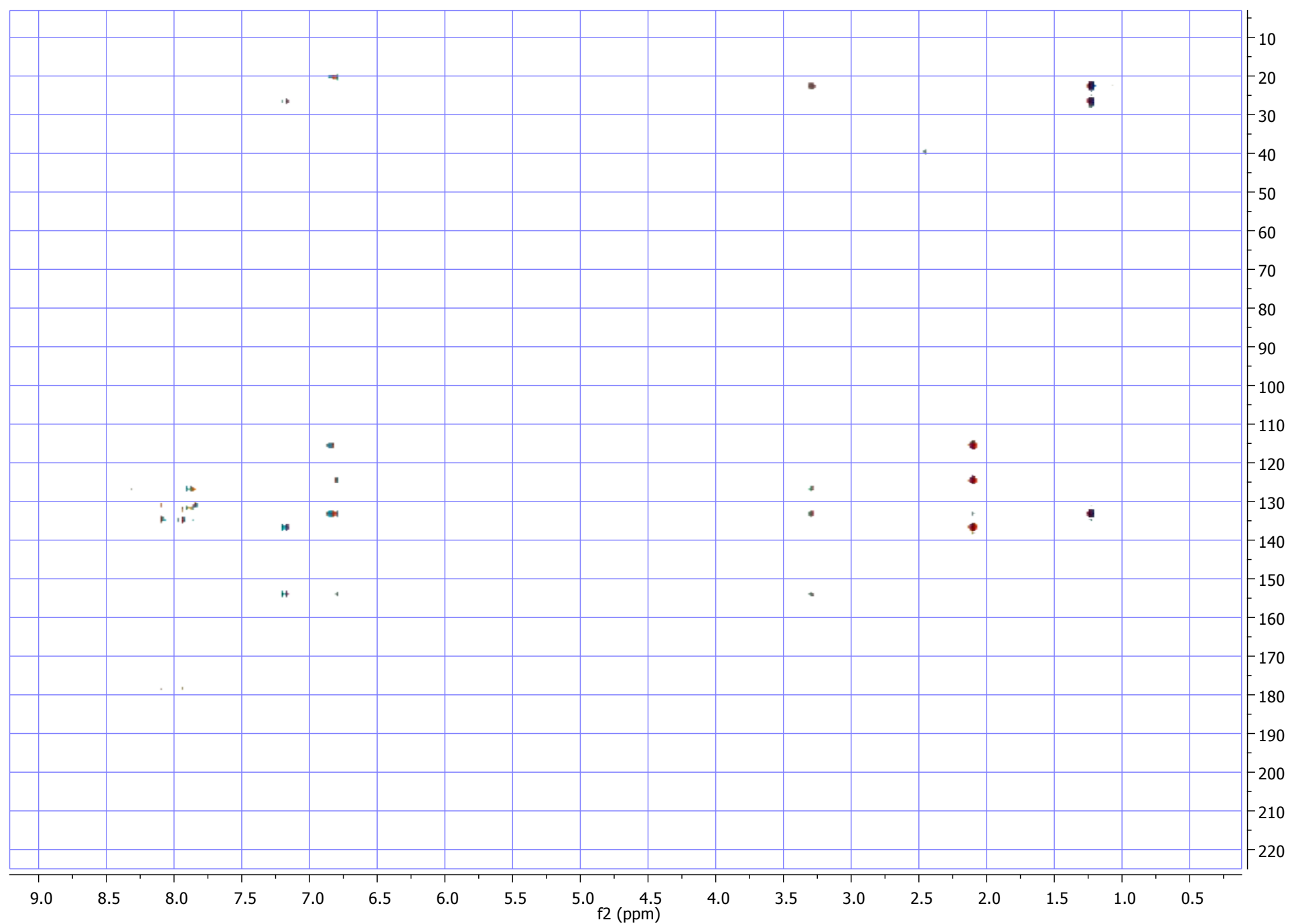

Figure S10 - HMBC spectrum (400 MHz, DMSO- $d_{6}$ ) of the compound (2-chloro-3-(2-isopropyl-5-methylphenoxy)naphthalene-1,4-dione) (2b). 


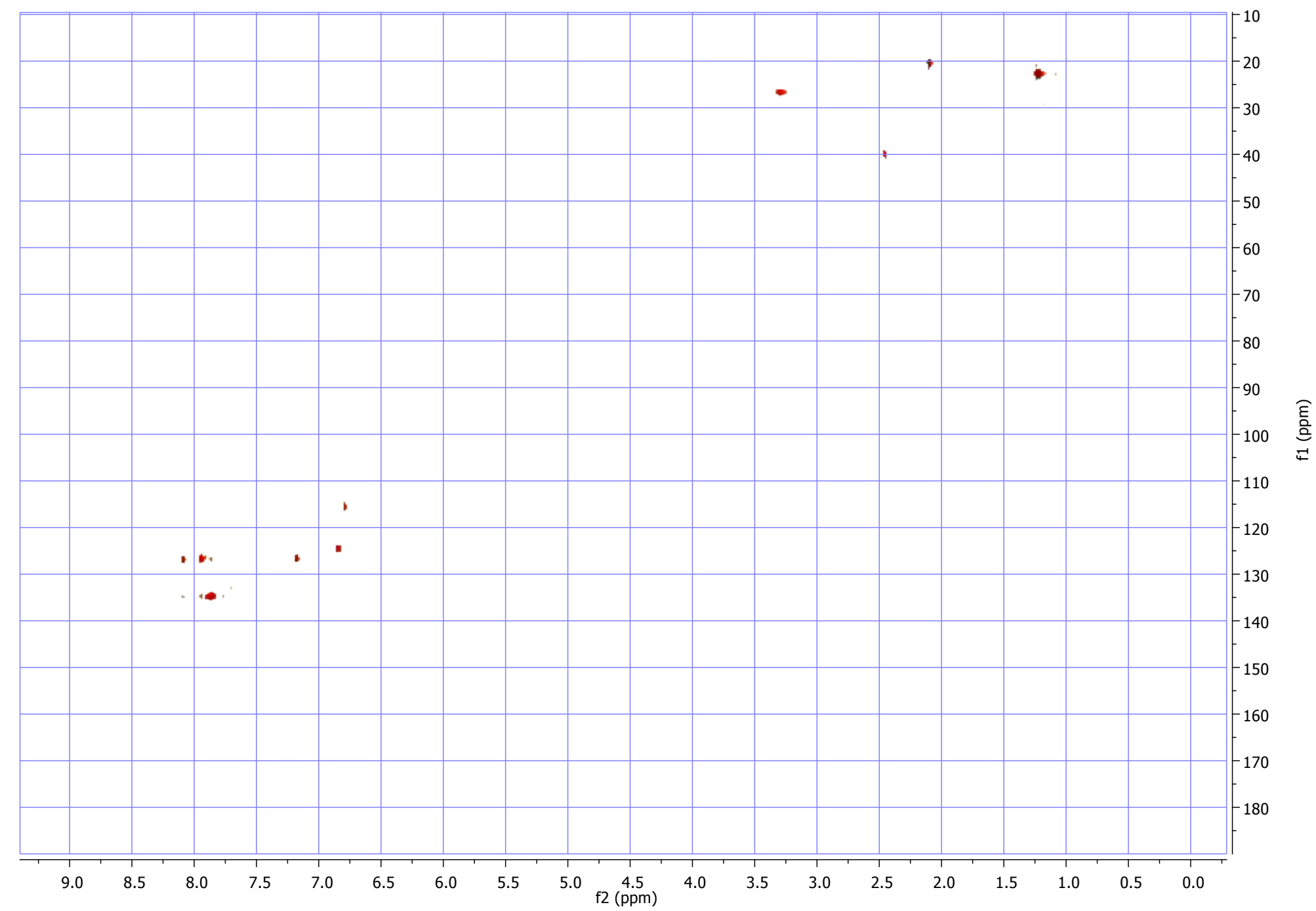

Figure S11 - HSQC spectrum (400 MHz, DMSO- $d_{6}$ ) of the compound (2-chloro-3-(2-isopropyl-5-methylphenoxy)naphthalene-1,4-dione) (2b). 


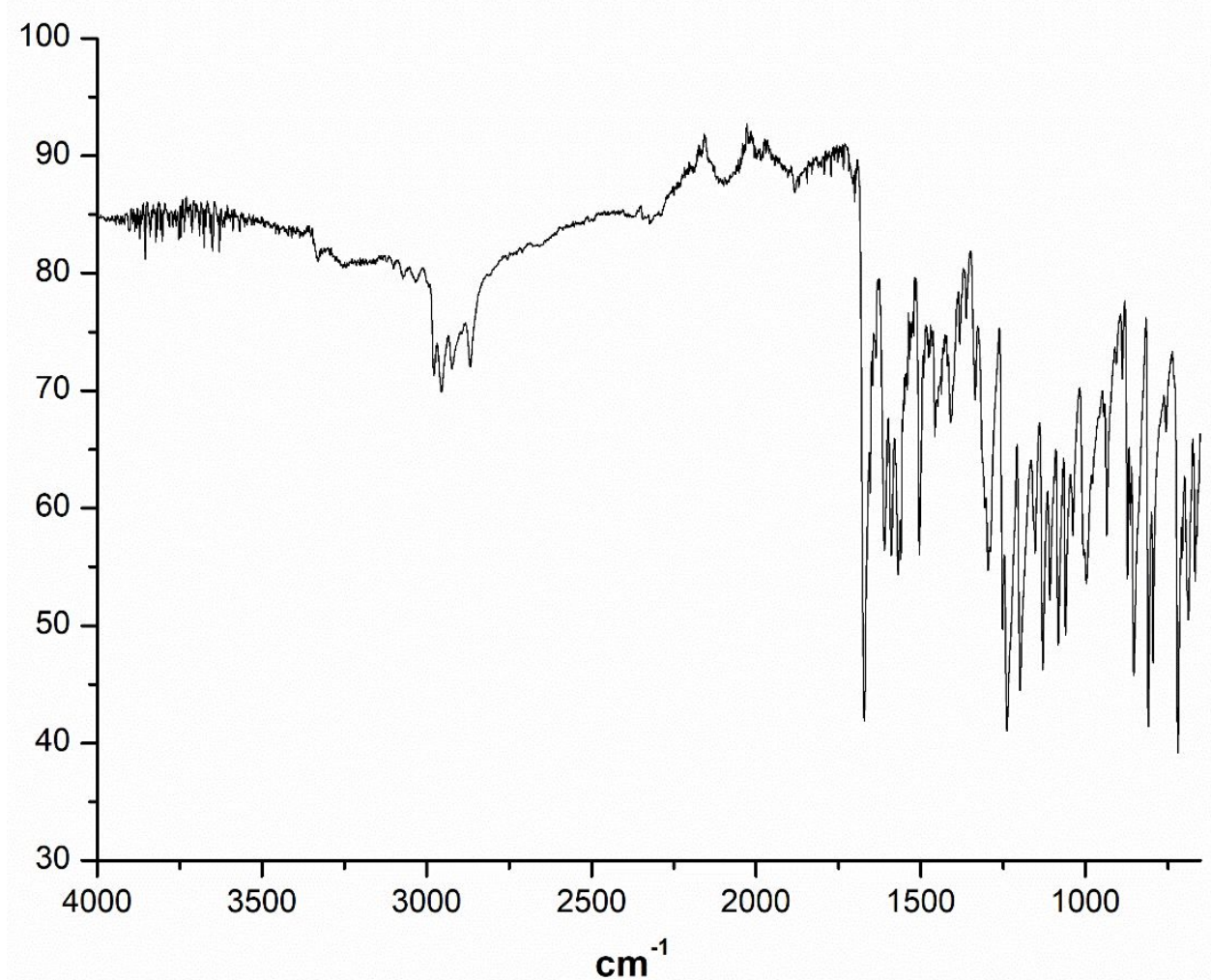

Figure S12. IR spectrum of compound 2b. 


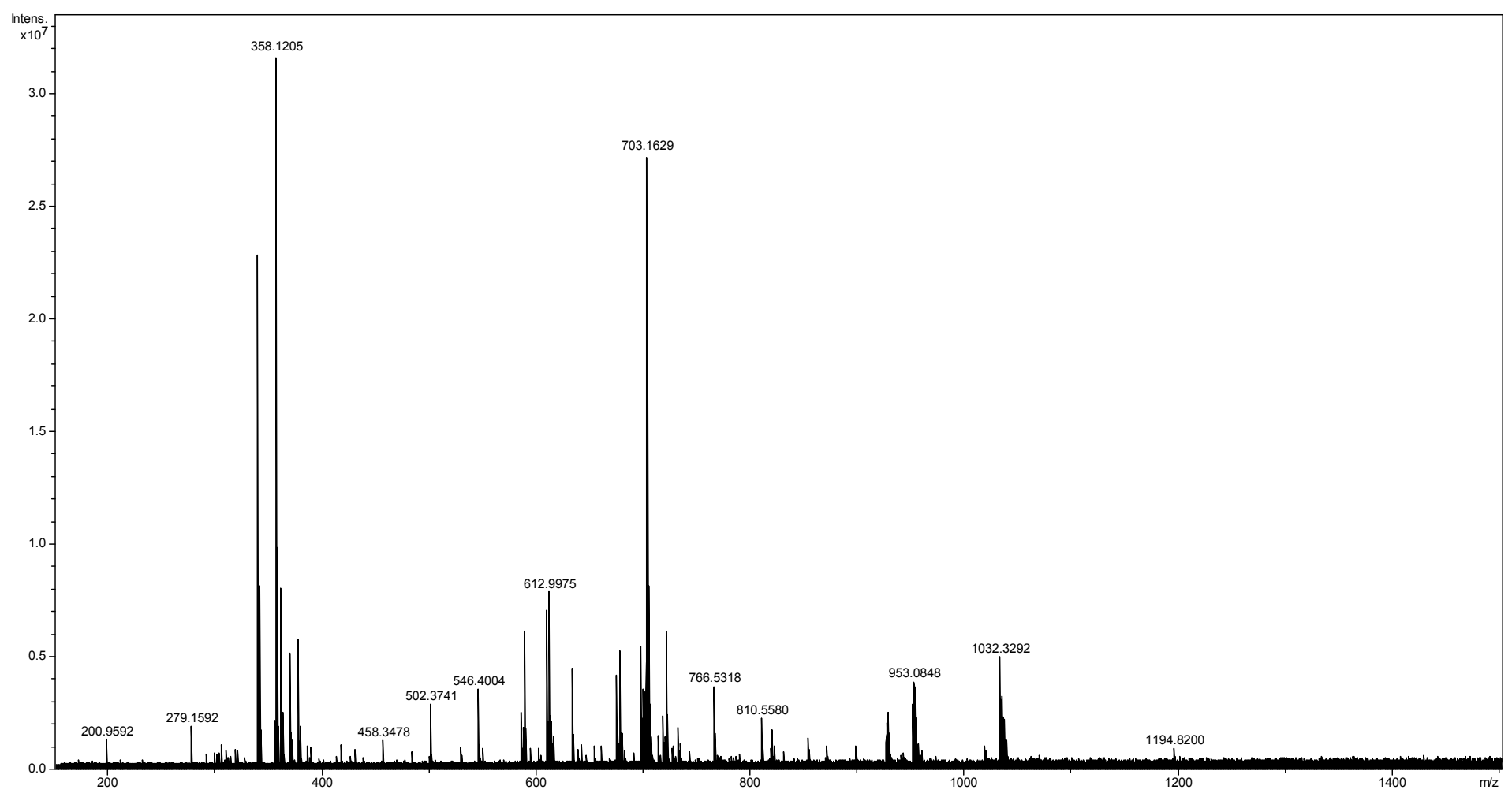

Figure S13. HRMS spectrum of compound $\mathbf{2 b}$. 


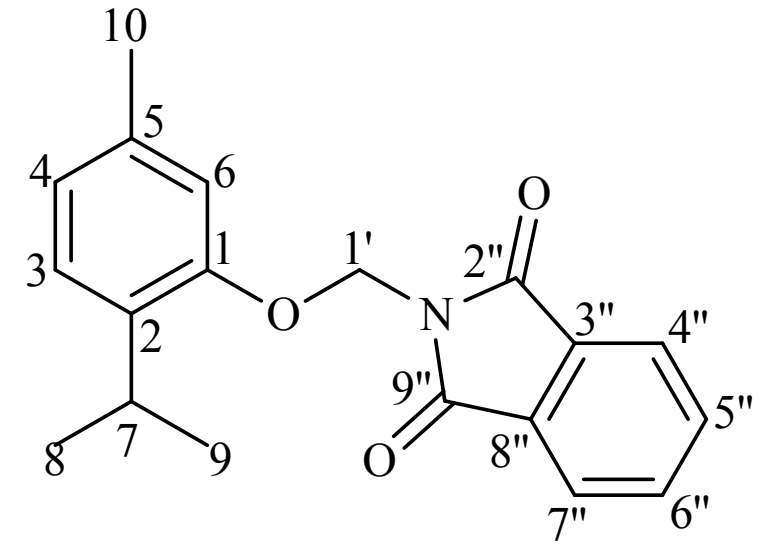

2c

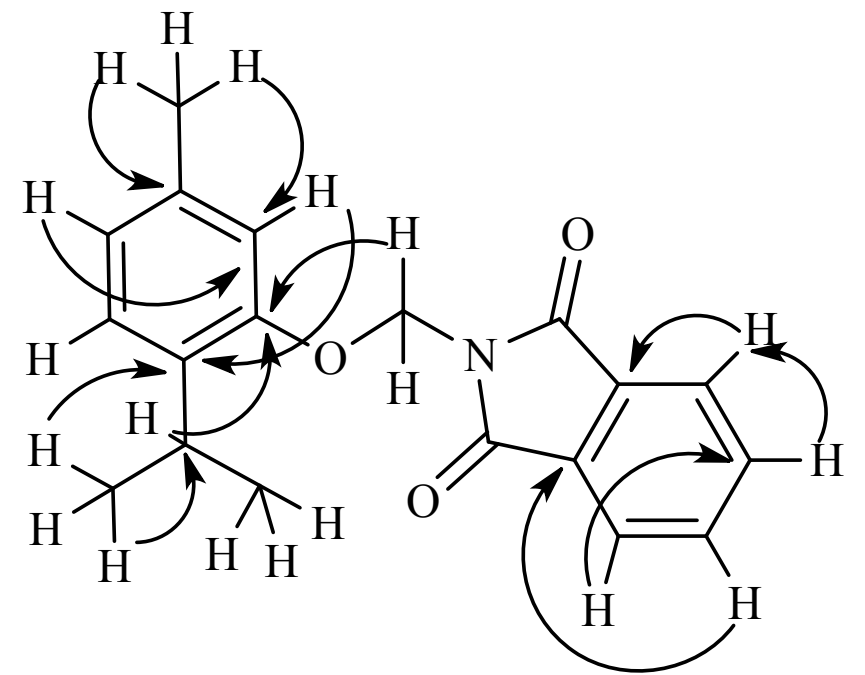

Figure S14. Compound 2c and main HMBC correlation $J$ in $\mathrm{Hz}$.
${ }^{1}$ H NMR (400 MHz, $1.14\left(6 \mathrm{H}, \mathrm{d}, \mathrm{CH}_{3}-8\right.$ and $\left.\mathrm{CH}_{3}-9\right)$; $2.33(3 \mathrm{H}, \mathrm{s}$ $\left.\mathrm{CDCl}_{3}\right)$ CH $\left.\mathrm{CH}_{2}-1{ }^{\prime}\right) ; 6.81\left(1 \mathrm{H}, \mathrm{d}, J_{4,3} 7.82, \mathrm{H}-4\right) ; 6.99(1 \mathrm{H}, \mathrm{s}$, H-6); 7.09 (1H, d, $\left.J_{3,4} 7.82, \mathrm{H}-3\right) ; 7.73-7.76(2 \mathrm{H}$, $\mathrm{m}, J_{5}, 7^{\prime}, 8.21, \mathrm{H}-5$ "' and H-6"'); 7.89-7.91 (2H, m, $J_{4},{ }^{\prime}, 8.21, \mathrm{H}-4$ '" and H-7'").

${ }^{13}$ C NMR (100 MHz, 21.18 (C-10); 22.94 (C-8, C-9); 26.42 (C-7); $\left.\mathrm{CDCl}_{3}\right)$ $65.70\left(\mathrm{C}^{\prime} \mathbf{1}^{\prime}\right) ; 115.03$ (C-6); $123.14(\mathrm{C}-4) ; 123.79$ (C-4', and C-7''); 126.23 (C-3); 131.83 (C-3'' and $\mathrm{C}-8$ ' '); 134.46 (C-2); 135.46 (C-5' ' and C6',); 136.43 (C-5); 153.32 (C-1); 167.79 (2', and 9').

Infra-red

Mass spectrometry HRESIMS $[\mathrm{M}+\mathrm{Na}]^{+}$Found: 332.1245. Calc. for $\mathrm{C}_{19} \mathrm{H}_{19} \mathrm{NNaO}_{3}{ }^{+}: 332.1263$

Table S3. Spectroscopic data for compound 2c. 

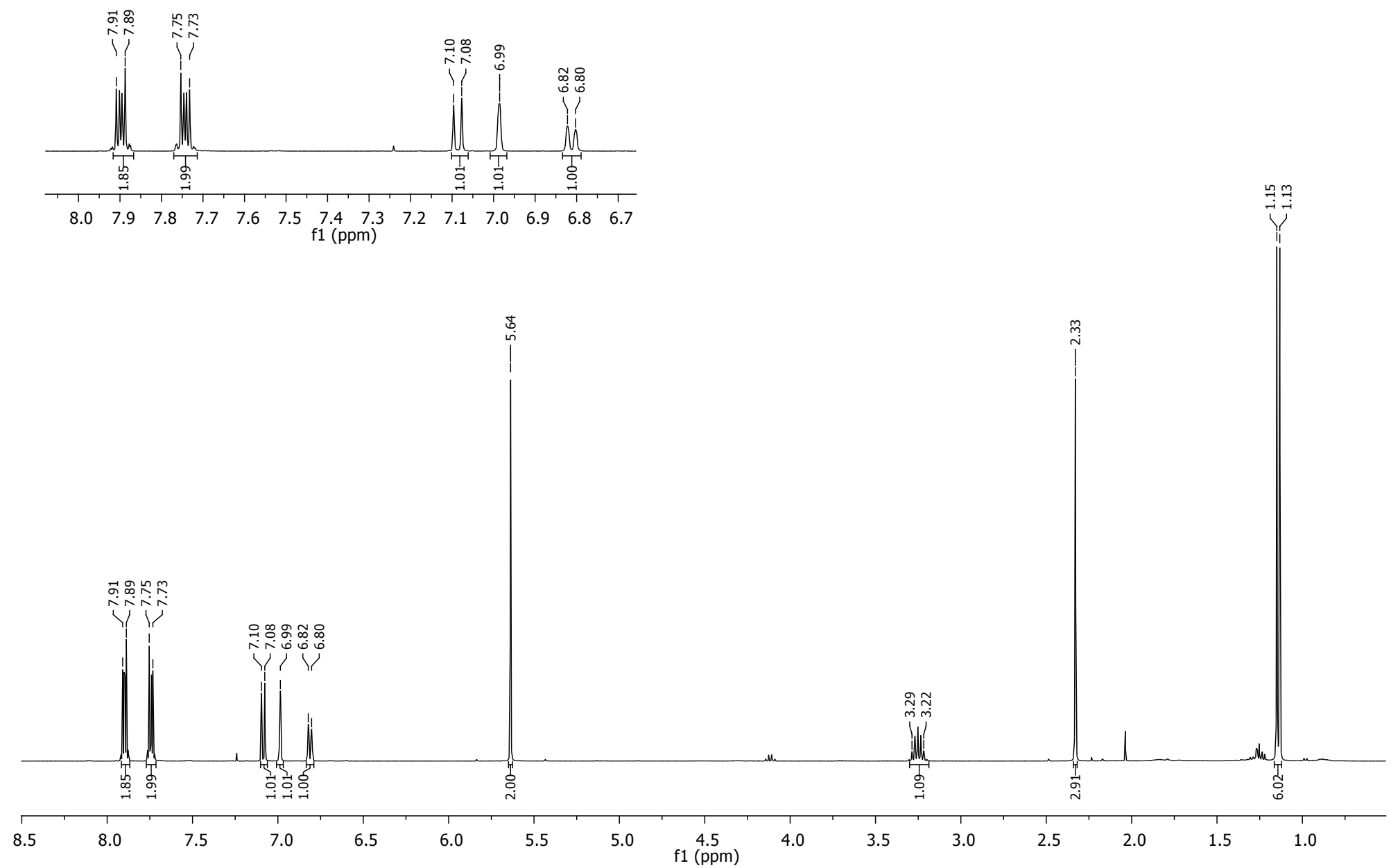

Figure S15. ${ }^{1} \mathrm{H}$ NMR spectrum (400 MHz, $\left.\mathrm{CDCl}_{3}\right)$ of compound (2-((2-isopropyl-5-methylphenoxy)methyl)-1H-indene-1,3(2H)-dione) (2c). 


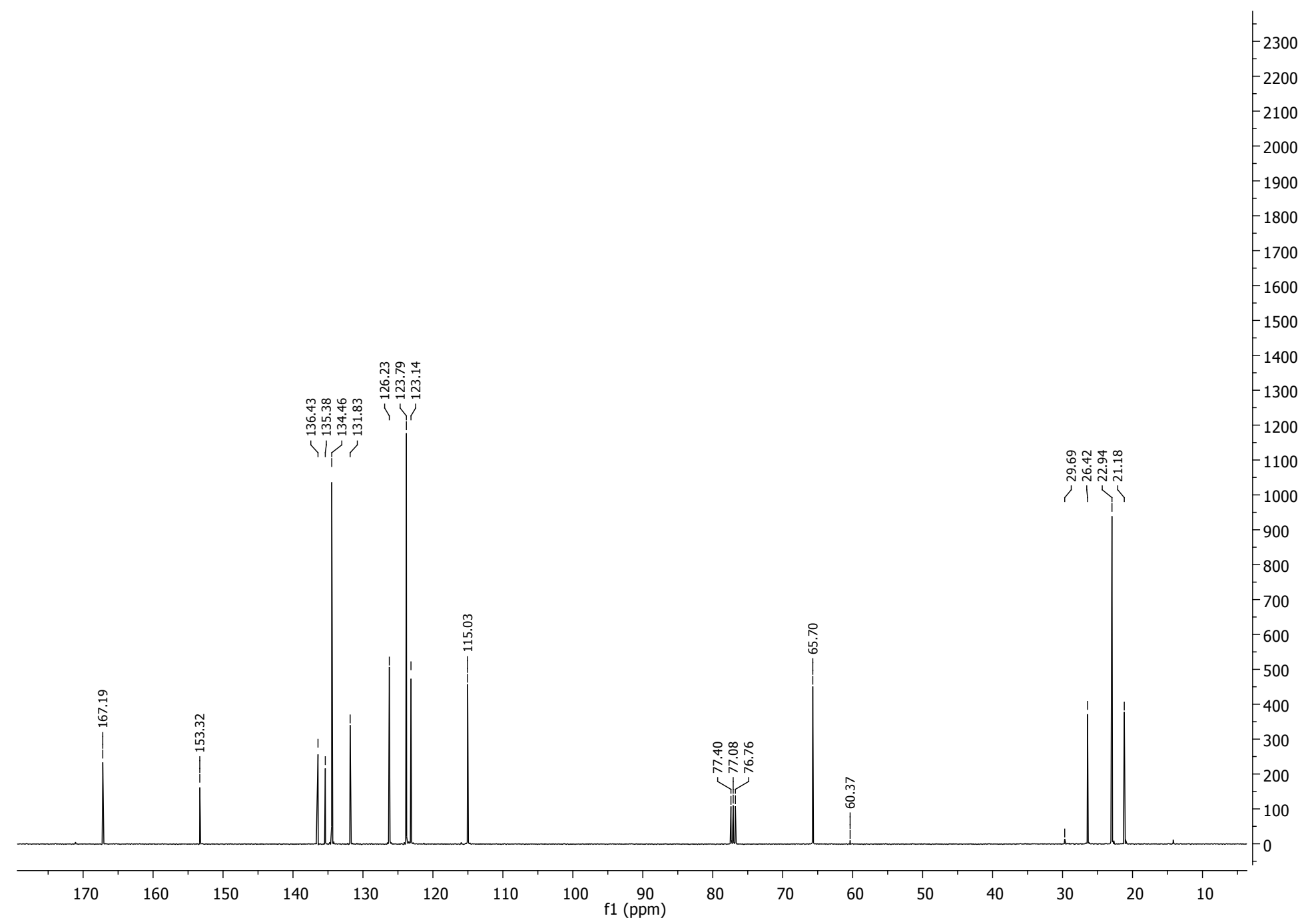

Figure S16. ${ }^{13} \mathrm{C}$ NMR spectrum (100 MHz, $\left.\mathrm{CDCl}_{3}\right)$ of compound (2-((2-isopropyl-5-methylphenoxy)methyl)-1H-indene-1,3(2H)-dione) (2c). 


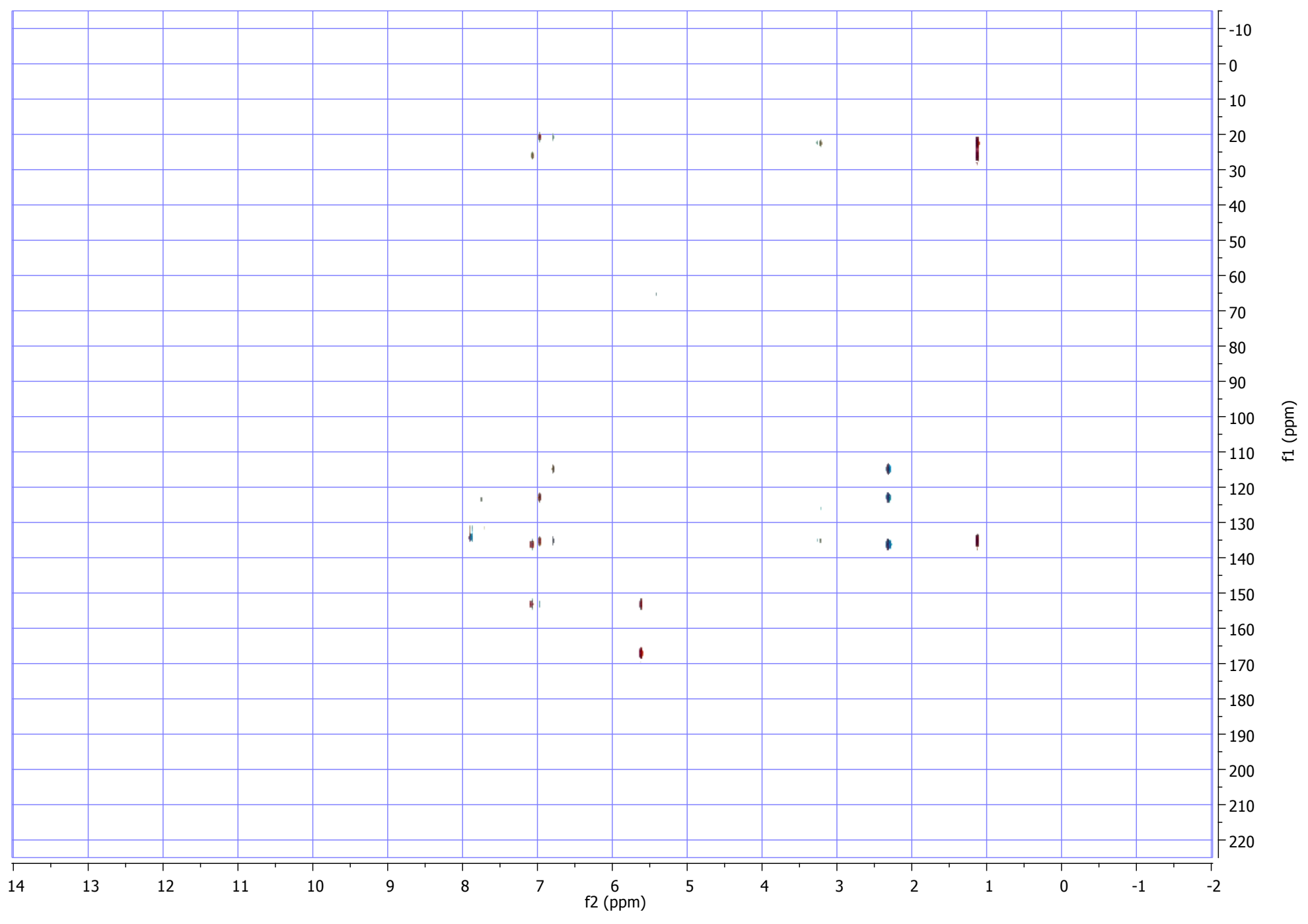

Figure S17 - HMBC spectrum (400 MHz, $\mathrm{CDCl}_{3}$ ) of the compound (2-((2-isopropyl-5-methylphenoxy)methyl)-1H-indene-1,3(2H)-dione) (2c). 


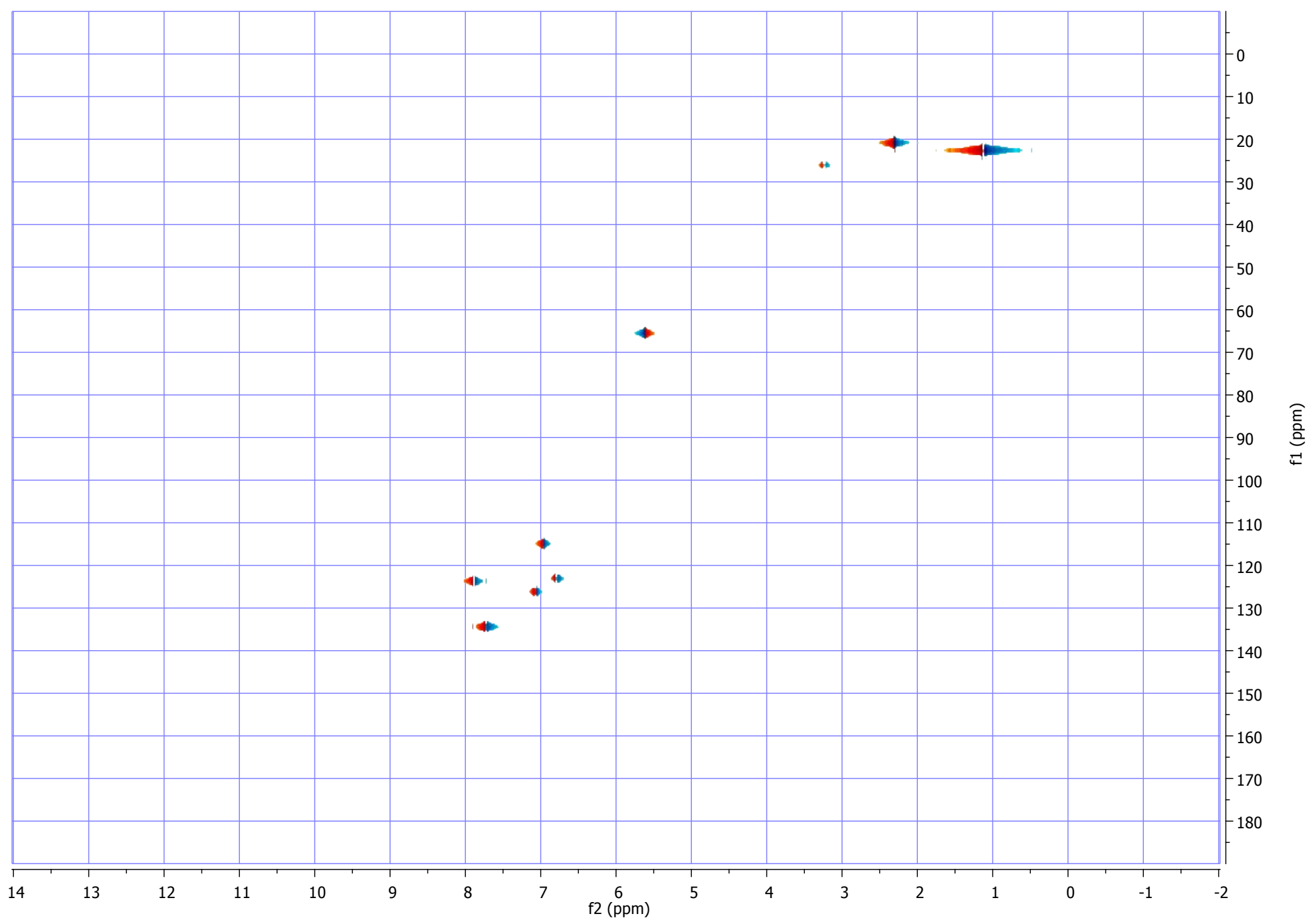

Figure S18. HSQC spectrum (400 MHz, $\left.\mathrm{CDCl}_{3}\right)$ of the compound (2-((2-isopropyl-5-methylphenoxy)methyl)-1H-indene-1,3(2H)-dione) (2c). 
Figure S19. IR spectrum of compound 2c 


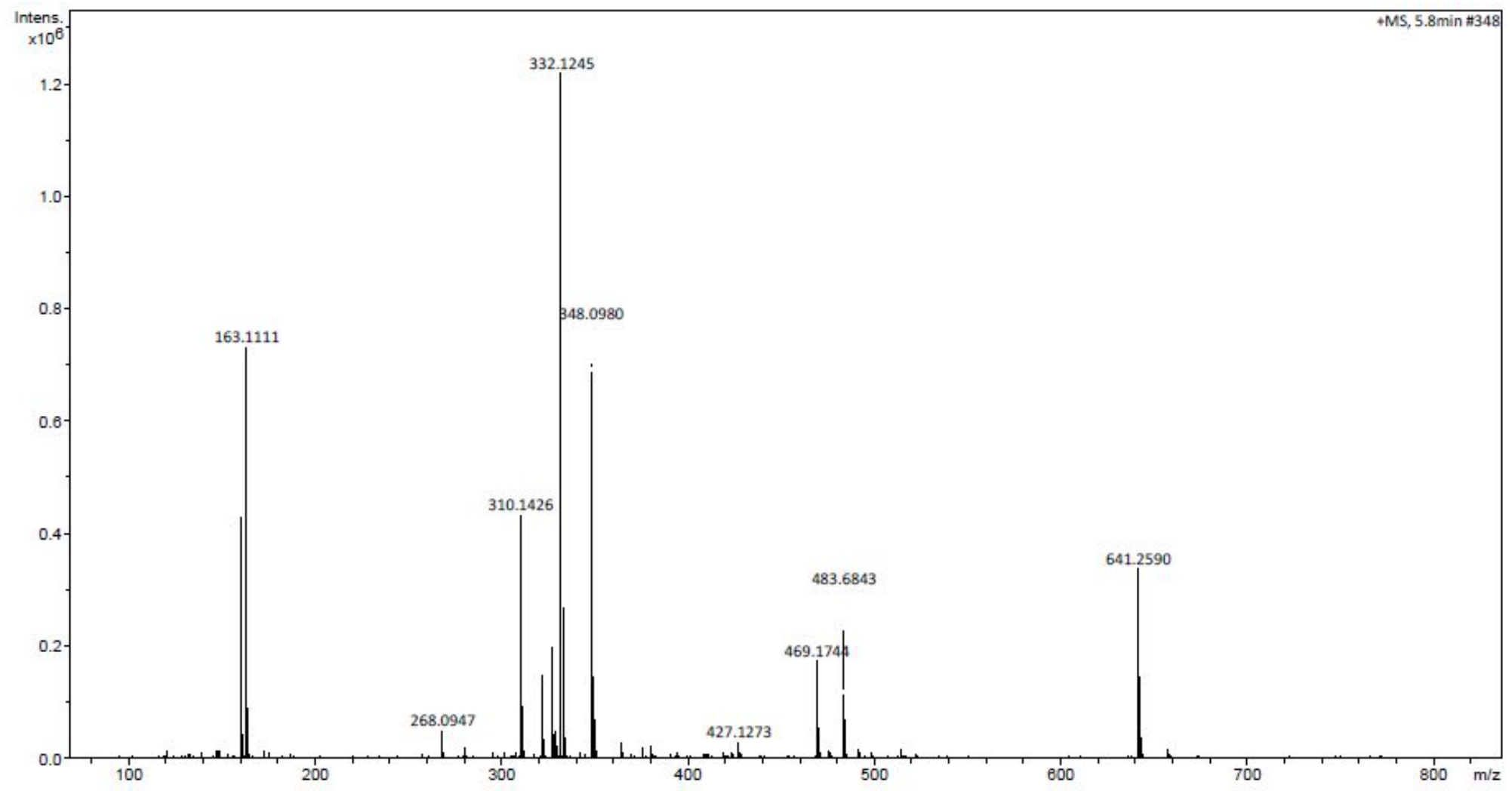

Figure S20. HRMS spectrum of compound 2c. 

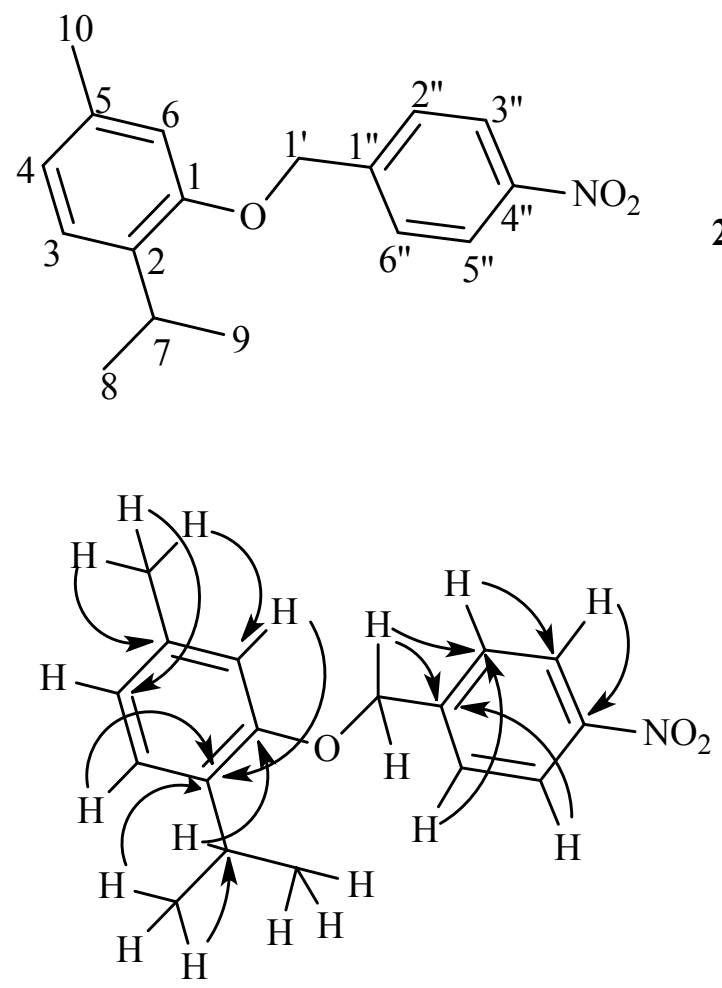

Figure S21. Compound 2d and main HMBC correlation $J$ in $\mathrm{Hz}$.
${ }^{1}$ H NMR (400 MHz, $1.25\left(6 \mathrm{H}, \mathrm{d}, \mathrm{CH}_{3}-8\right.$ and $\left.\mathrm{CH}_{3}-9\right) ; 2.31(3 \mathrm{H}, \mathrm{s}$, $\left.\mathrm{CDCl}_{3}\right)$ $\left.\mathrm{CH}_{3}-10\right) ; 3.34-3.40(1 \mathrm{H}, \mathrm{m}, \mathrm{H}-7) ; 5.17(2 \mathrm{H}, \mathrm{s}$, $\left.\mathrm{CH}_{2}-1^{\prime}\right) ; 6.69(1 \mathrm{H}, \mathrm{s}, \mathrm{H}-6) ; 6.80\left(1 \mathrm{H}, \mathrm{d}, J_{4,3} 7.78\right.$, $\mathrm{H}-4) ; 7.15\left(1 \mathrm{H}, \mathrm{d}, J_{3,4} 7.78, \mathrm{H}-3\right) ; 7.62(2 \mathrm{H}, \mathrm{d}$, $J_{2},{ }_{3}, 8.69, \mathrm{H}-2$ ', and $\left.\mathrm{H}-6{ }^{\prime}\right) ; 8.26\left(2 \mathrm{H}, \mathrm{d}, J_{3}{ }^{\prime \prime}, 6^{\prime}\right.$, 8.69, H-3'" and H-5'").

${ }^{13}$ C NMR (100 MHz, 21.33 (C-10); 22.81 (C-8, C-9); 26.70 (C-7); $\left.\mathrm{CDCl}_{3}\right)$ $68.74\left(\mathrm{C}-1^{\prime}\right) ; 112.55(\mathrm{C}-6) ; 122.06(\mathrm{C}-4) ; 123.84$ (C-3', C-5' '); 126.25 (C-3); 127.34 (C-2', C6''); 134.32 (C-2); $136.51(\mathrm{C}-5) ; 145.11(\mathrm{C}-1$ ''); 147.50 (C-4”); 155.18 (C-1).

Infra-red $\left(\mathrm{cm}^{-1}\right)$ 2962 (C-H in $\mathrm{CH}_{2}-1$ ', $\mathrm{CH}-7, \mathrm{CH}_{3}-8, \mathrm{CH}_{3}-9$, $\left.\mathrm{CH}_{3}-10\right) ; 1502$ and $1330\left(\mathrm{NO}_{2}\right.$ in $\mathrm{C}-4$ ''); 1257 and 1052 (ether in $\mathrm{CH}_{2}-1$ ' and $\mathrm{C}-1$ ).

Mass spectrometry HRESIMS $[\mathrm{M}+\mathrm{Na}]^{+}$Found: 308.1260 Calc. for $\mathrm{C}_{17} \mathrm{H}_{19} \mathrm{NNaO}_{3}{ }^{+}: 308.1263$

Table S4. Spectroscopic data for compound $\mathbf{2 d}$ 

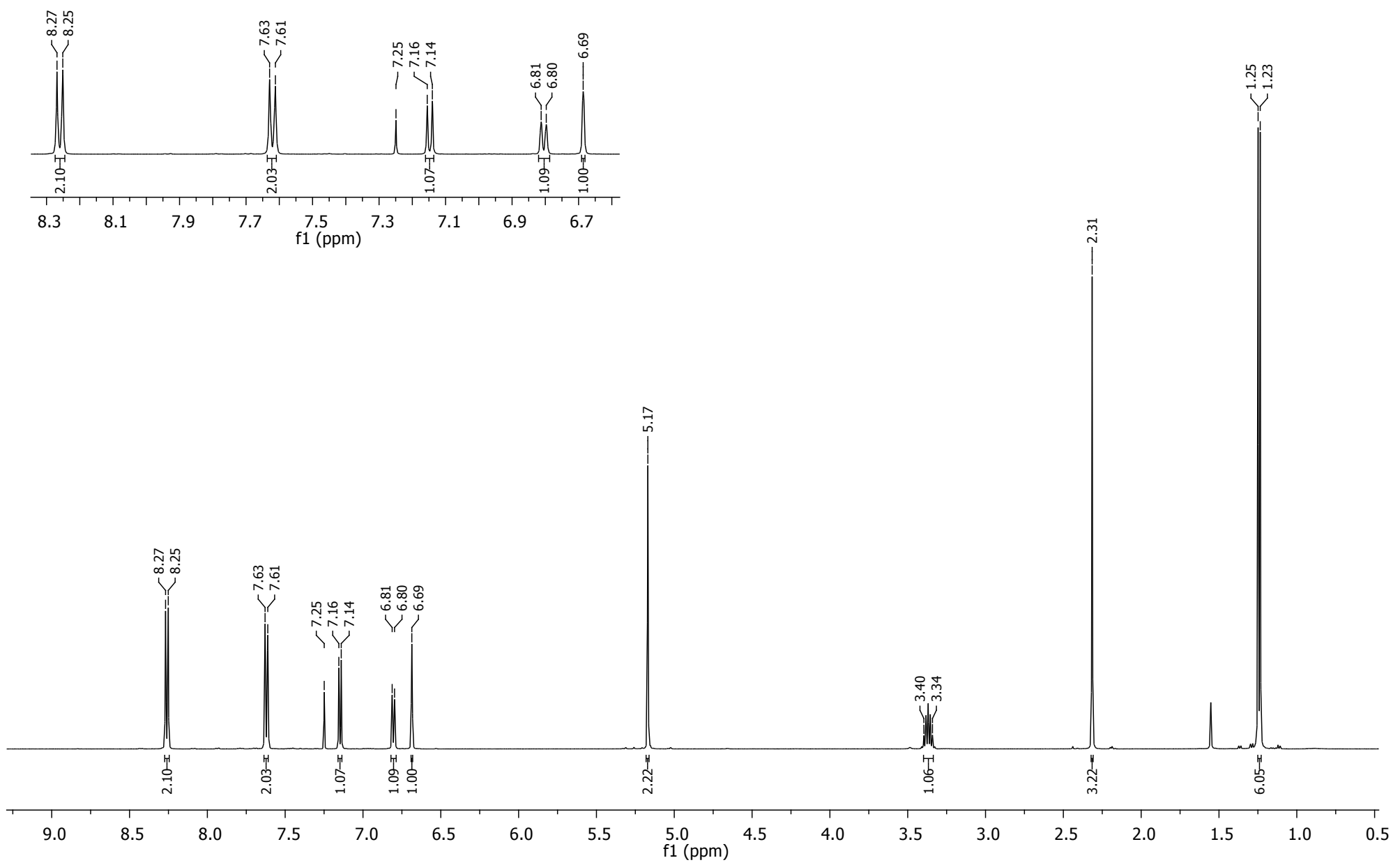

Figure S22. ${ }^{1} \mathrm{H}$ NMR spectrum (400 MHz, $\mathrm{CDCl}_{3}$ ) of compound (1-isopropyl-4-methyl-2-((4-nitrobenzyl)oxy)benzene) (2d). 


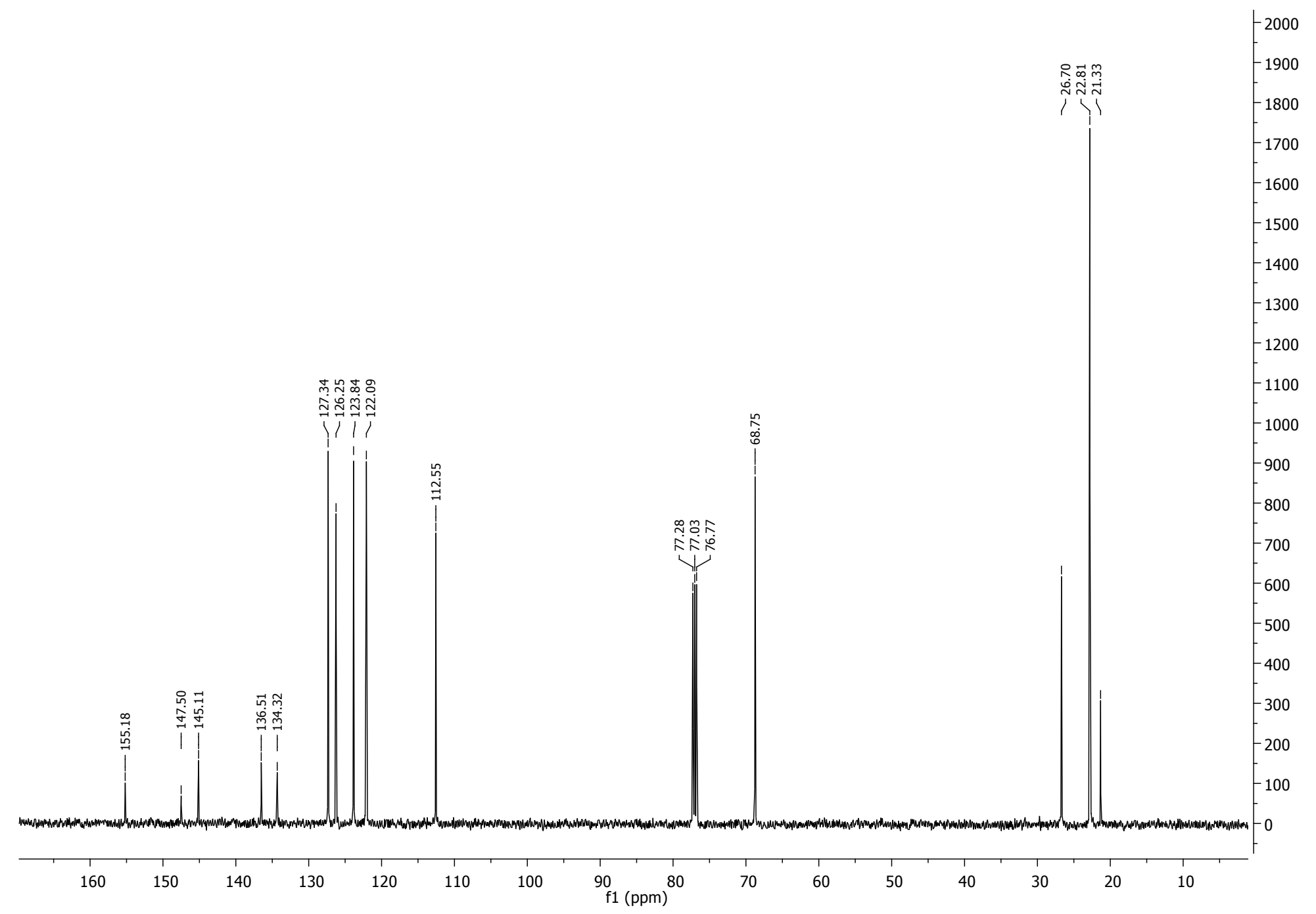

Figure S23. ${ }^{13} \mathrm{C}$ NMR spectrum (100 MHz, $\mathrm{CDCl}_{3}$ ) of compound (1-isopropyl-4-methyl-2-((4-nitrobenzyl)oxy)benzene) (2d). 


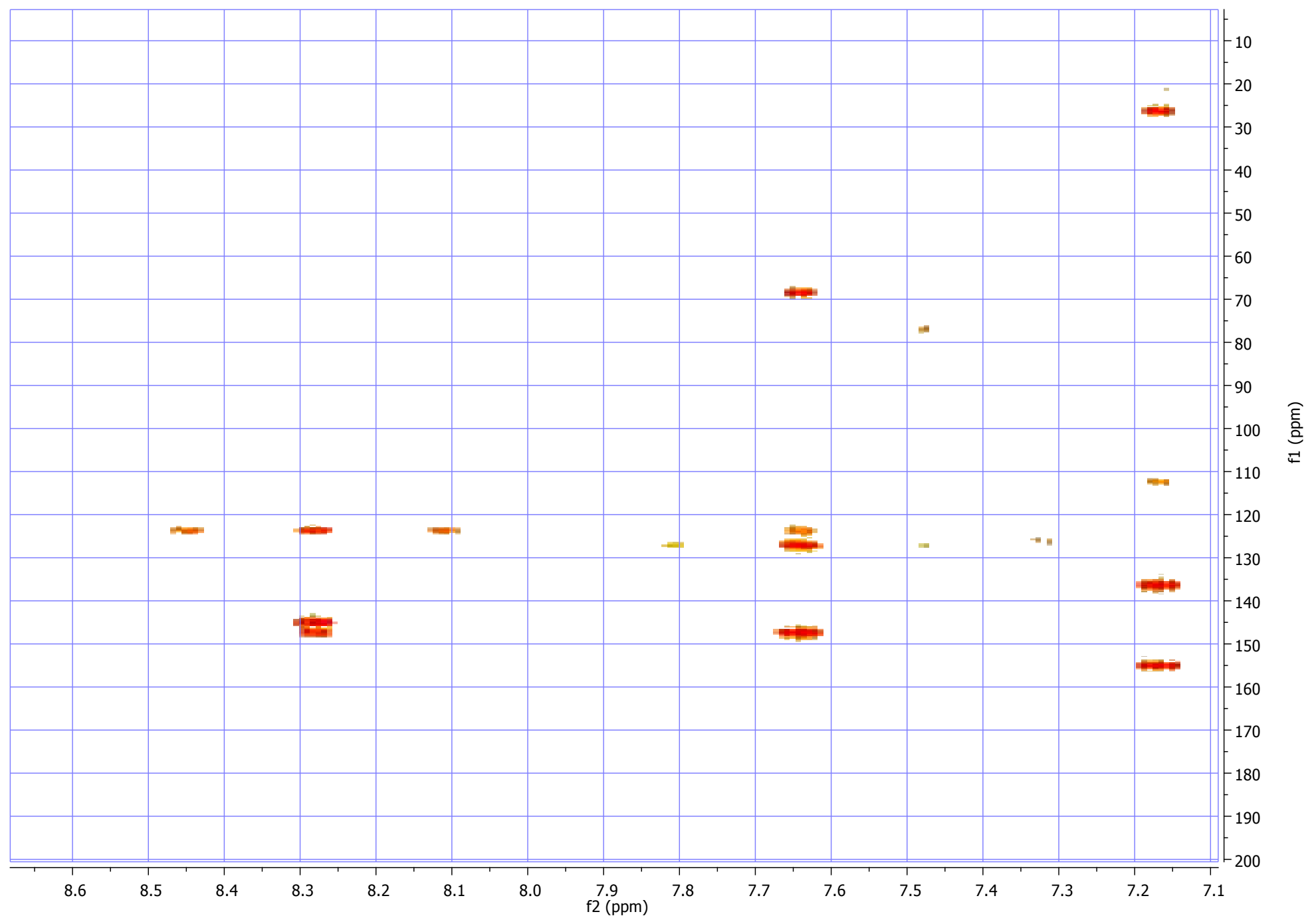

Figure S24 - HMBC spectrum (400 MHz, $\mathrm{CDCl}_{3}$ ) of the compound (1-isopropyl-4-methyl-2-((4-nitrobenzyl)oxy)benzene) (2d). 


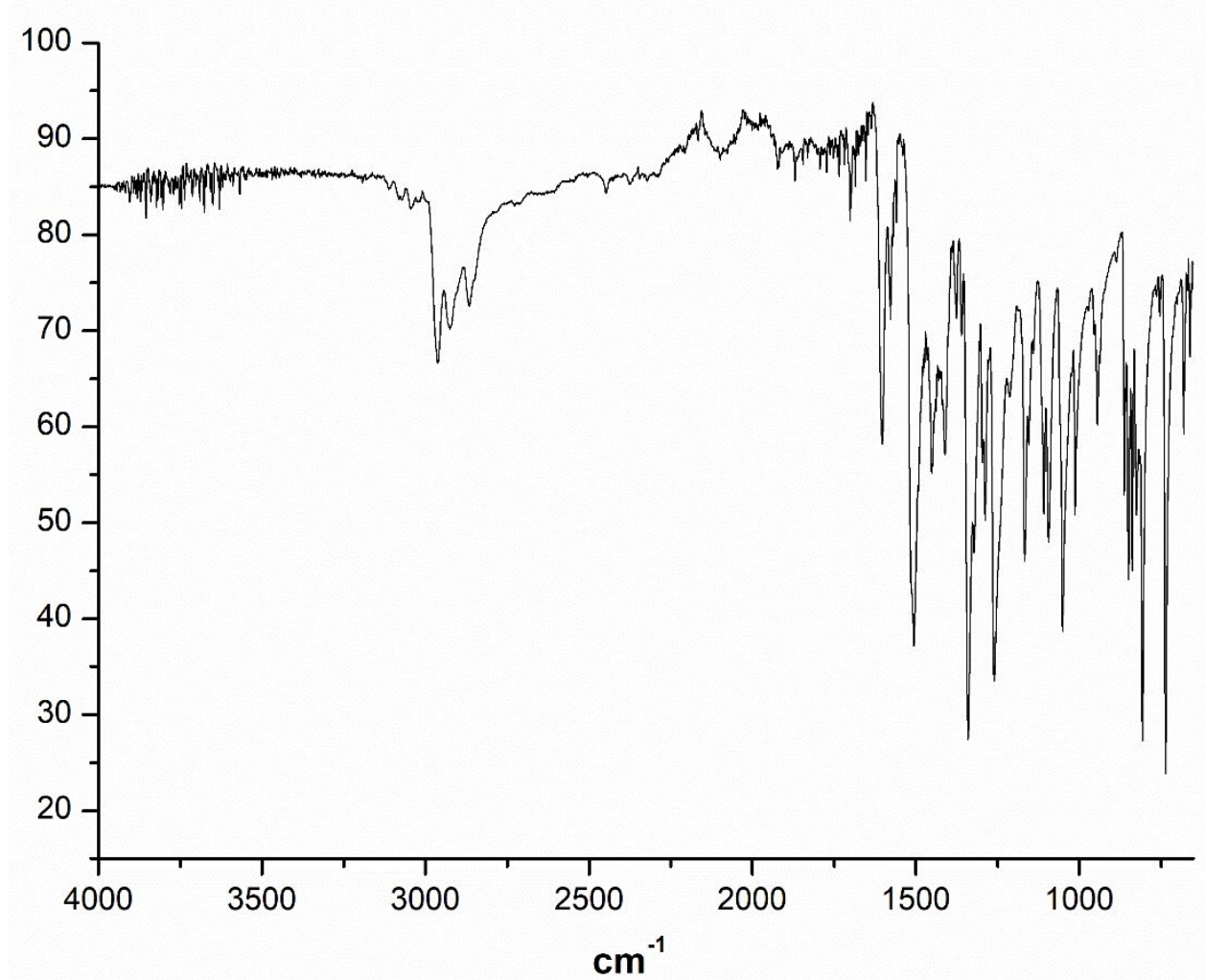

Figure S25. IR spectrum of compound 2d. 


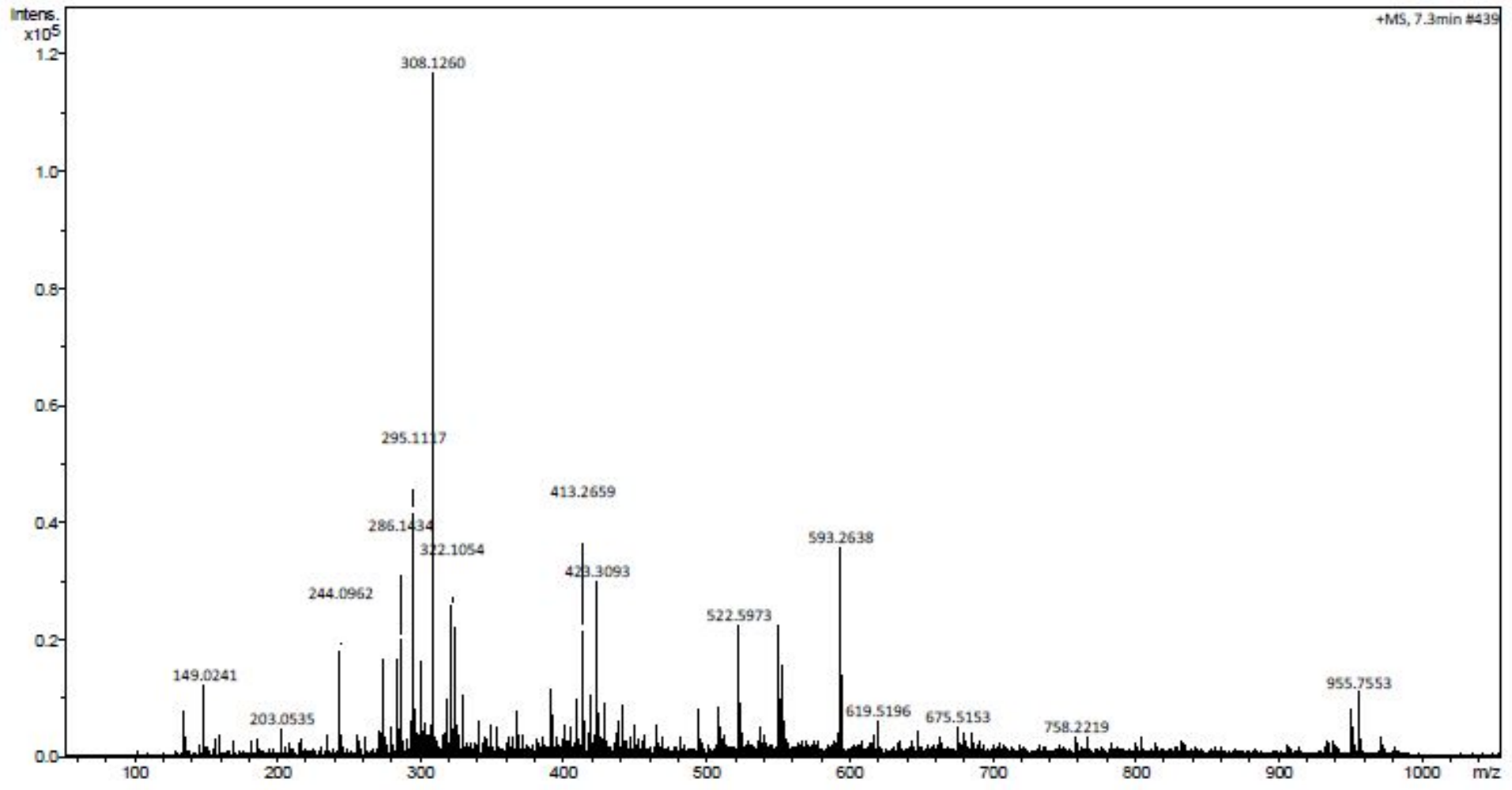

Figure S26. HRMS spectrum of compound 2d. 


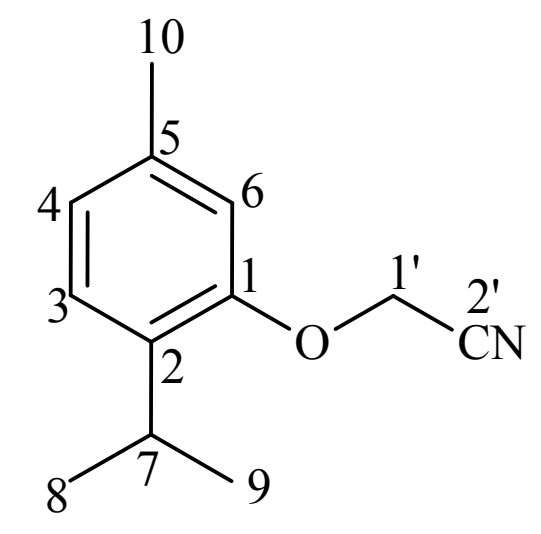

$2 \mathrm{e}$

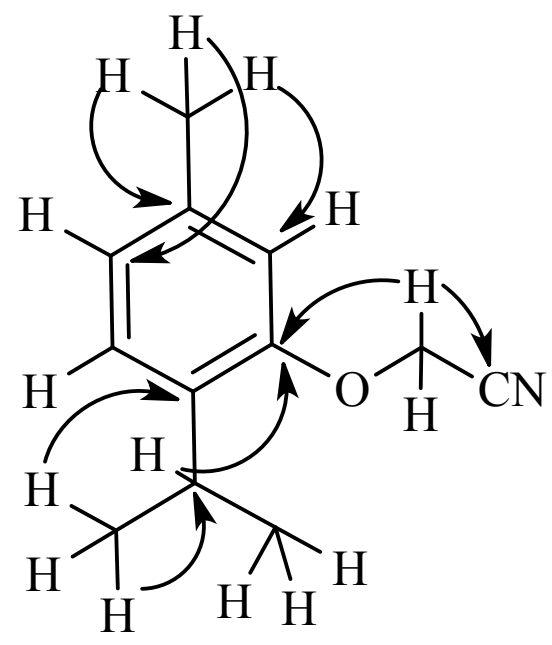

${ }^{1} \mathrm{H}$ NMR (400 MHz, $1.12\left(6 \mathrm{H}, \mathrm{d}, \mathrm{CH}_{3}-8\right.$ and $\left.\mathrm{CH}_{3}-9\right) ; 2.26(3 \mathrm{H}, \mathrm{s}$, DMSO- $\left.\left.d_{6}\right) \quad \mathrm{CH}_{3}-10\right) ; 3.11-3.18(1 \mathrm{H}, \mathrm{m}, \mathrm{H}-7) ; 5.13(2 \mathrm{H}, \mathrm{s}$ $\mathrm{CH}_{2}-1$ '); $6.82\left(1 \mathrm{H}, \mathrm{d}, J_{4,3} 7.82, \mathrm{H}-4\right) ; 6.89(1 \mathrm{H}, \mathrm{s}$, $\mathrm{H}-6) ; 7.11\left(1 \mathrm{H}, \mathrm{d}, J_{3,4} 7.82, \mathrm{H}-3\right)$.

${ }^{13}$ C NMR (100 MHz, 21.29 (C-10); 23.12 (C-8, C-9); 26.44 (C-7); DMSO- $d_{6}$ ) 54.23 (C-1'); 113.64 (C-6); 117.29 (C-2'); 123.47 (C-4); 126.63 (C-3); 134.29 (C-2); $136.63(\mathrm{C}-5) ; 153.84(\mathrm{C}-1)$.

Infra-red $\left(\mathrm{cm}^{-1}\right)$

2960 (C-H in $\mathrm{CH}_{2}-1$ ', $\left.\mathrm{CH}_{3}-8, \mathrm{CH}_{3}-9, \mathrm{CH}_{3}-10\right)$; 2871 (C-H in $\mathrm{CH}-7) ; 1239$ and 1048 (ether in $\mathrm{CH}_{2}-1$ ' and $\left.\mathrm{C}-1\right)$.

Mass spectrometry HRESIMS $[\mathrm{M}+\mathrm{Na}]^{+}$Found: 212.1045. Calc. for $\mathrm{C}_{12} \mathrm{H}_{15} \mathrm{NaO}^{+}: 212.1046$.

Table S5. Spectroscopic data for compound $\mathbf{2 e .}$

Figure S27. Compound 2e and main HMBC correlation $J$ in $\mathrm{Hz}$. 

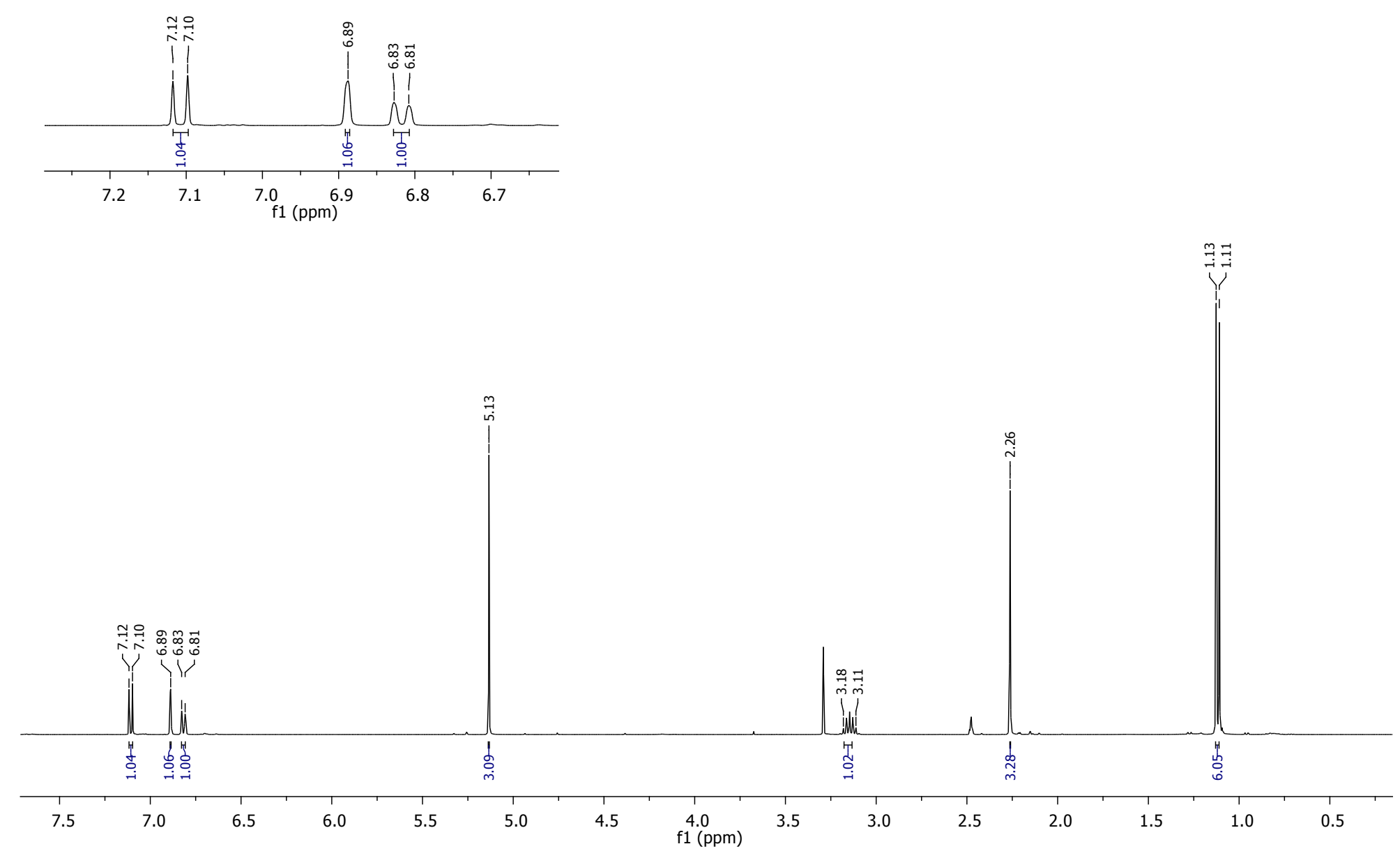

Figure S28. ${ }^{1} \mathrm{H}$ NMR spectrum (400 MHz, DMSO- $d_{6}$ ) of compound (2-(2-isopropyl-5-methylphenoxy)acetonitrile) (2e). 


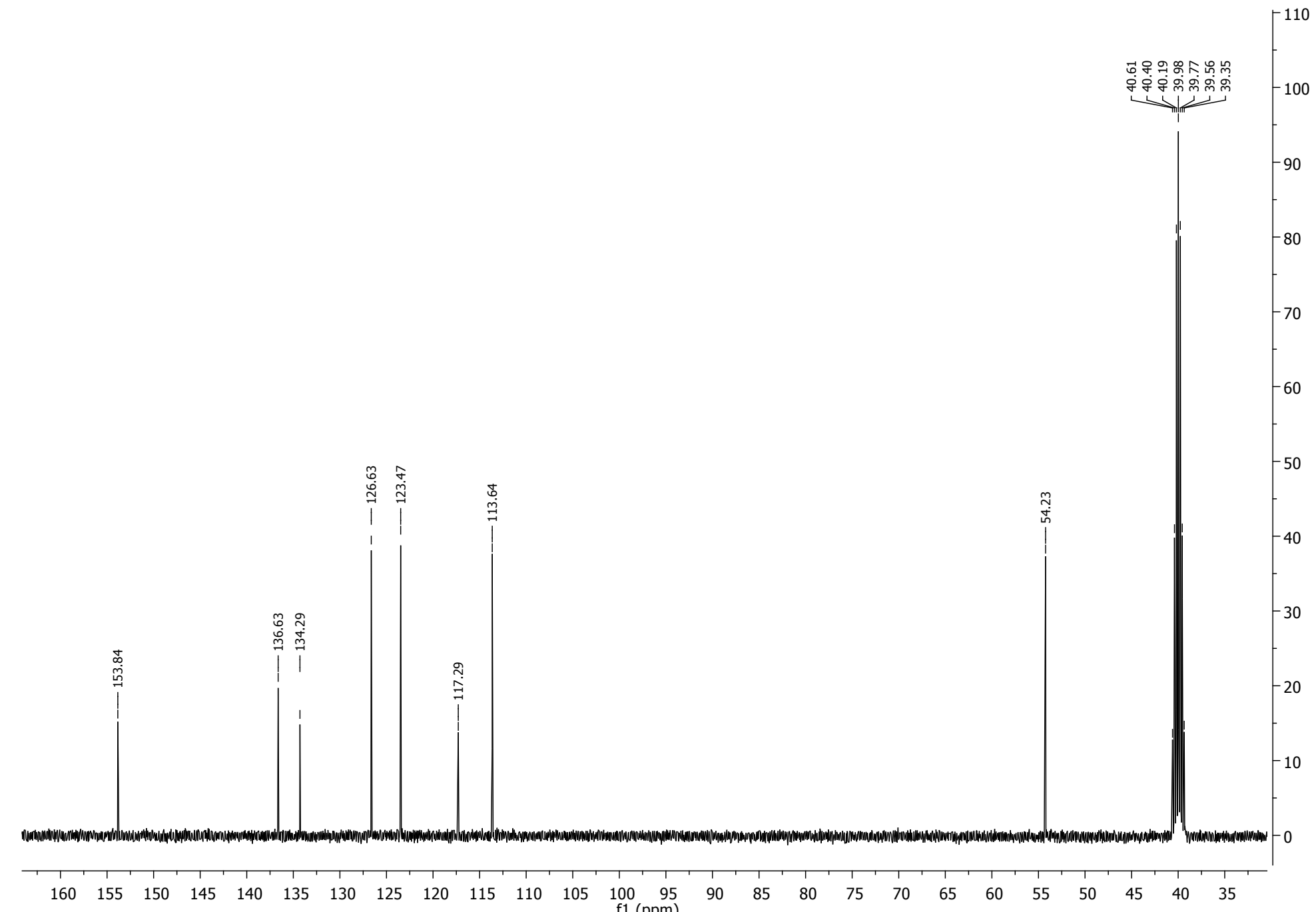

Figure S29. ${ }^{13} \mathrm{C}$ NMR spectrum (100 MHz, DMSO- $d_{6}$ ) of compound (2-(2-isopropyl-5-methylphenoxy)acetonitrile) (2e). 


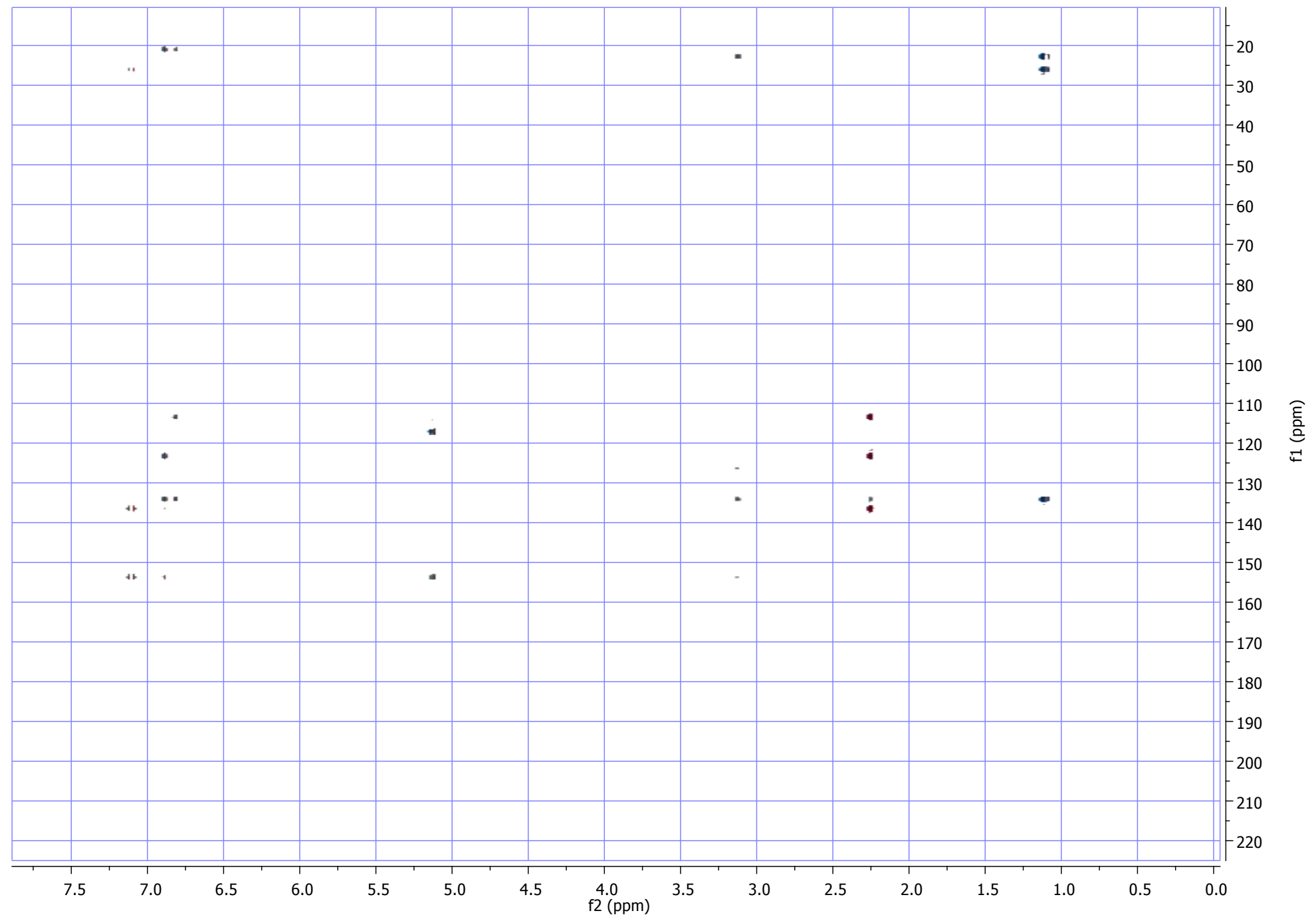

Figure S30 - HMBC spectrum (400 MHz, DMSO- $d_{6}$ ) of the compound (2-(2-isopropyl-5-methylphenoxy)acetonitrile) (2e). 


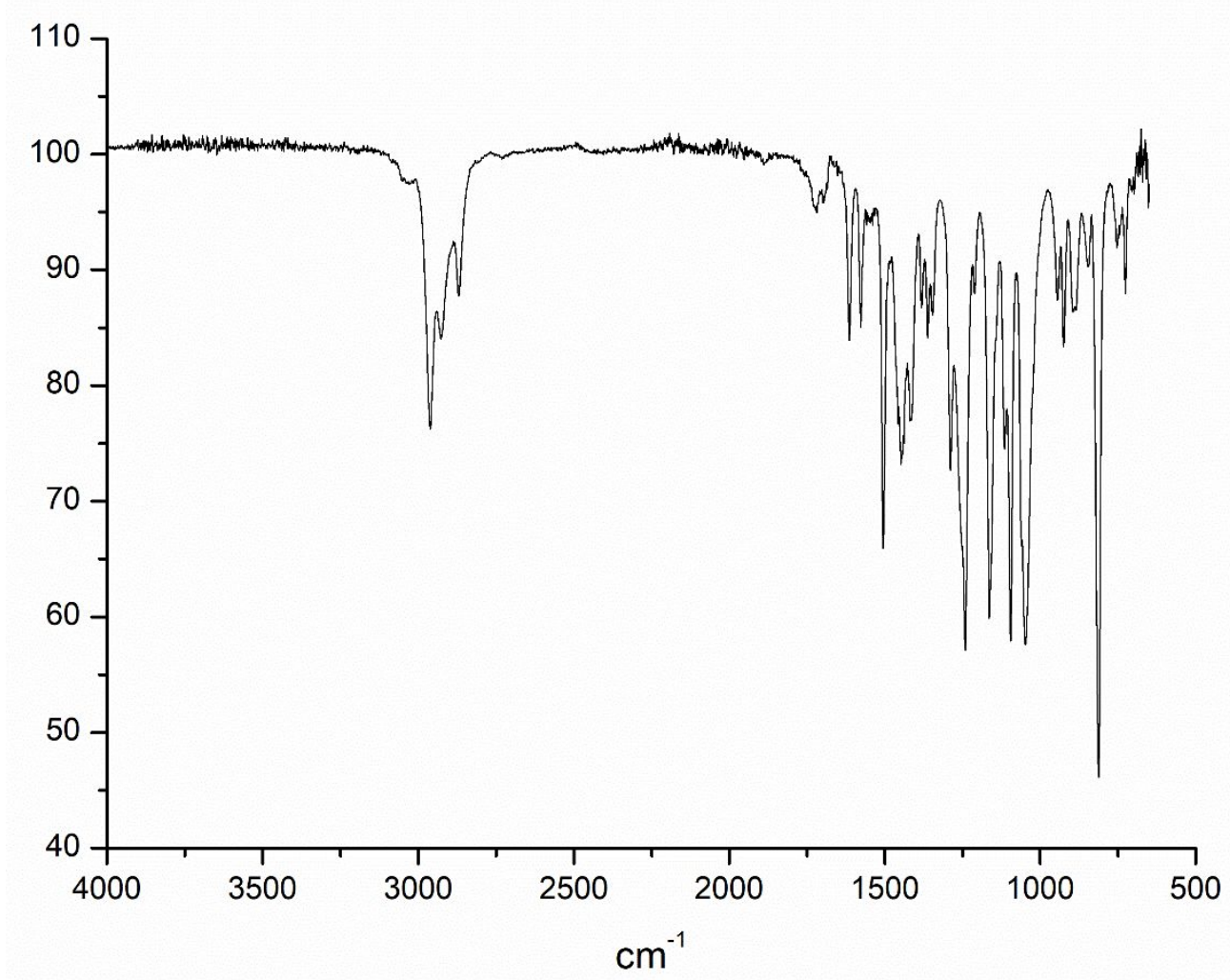

Figure S31. IR spectrum of compound 2e. 


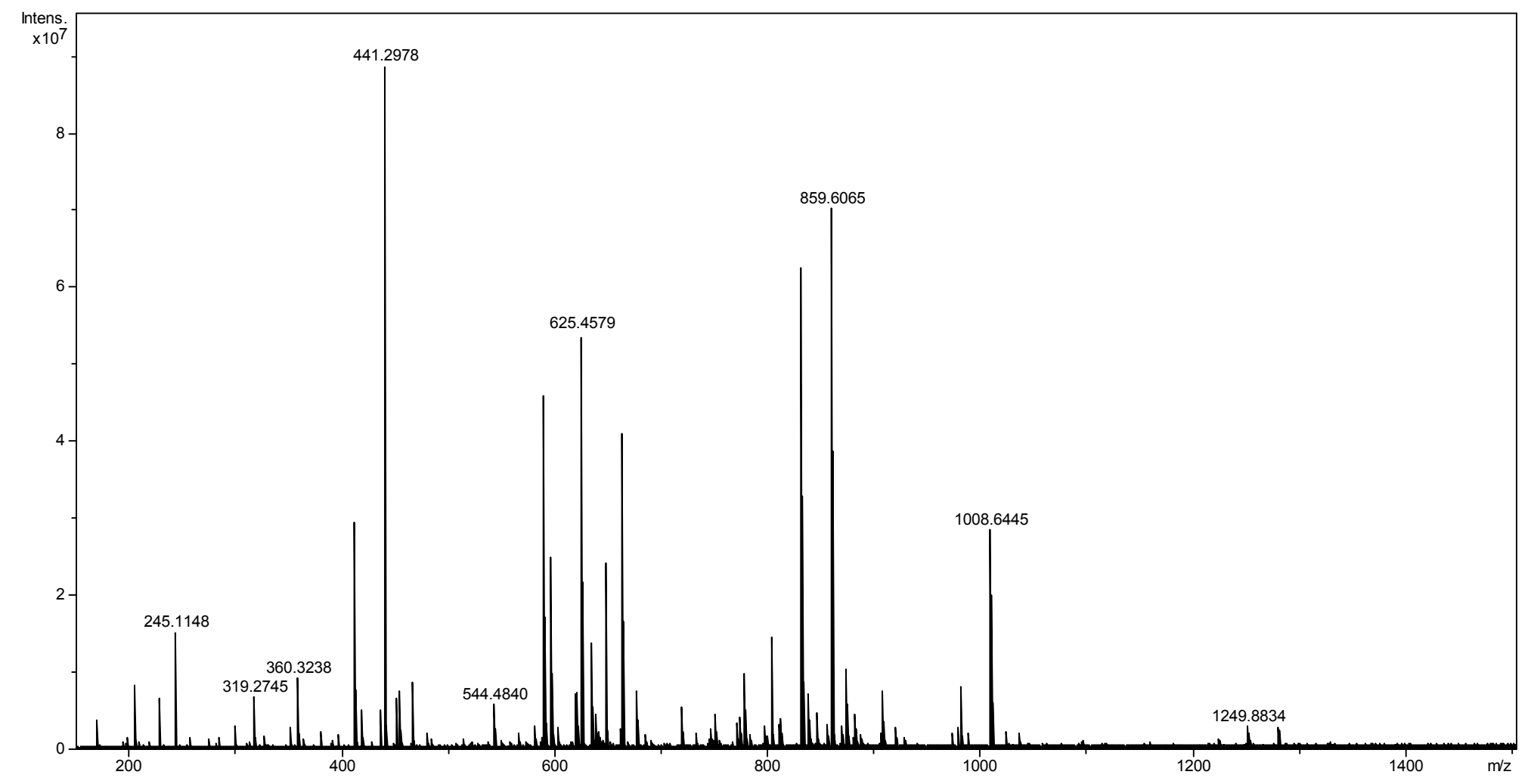

Figure S32. HRMS spectrum of compound 2e. 
<smiles>CC(C)c1ccc(Br)cc1OCC(C)c1ccccc1C[In]S(=O)(=O)c1ccccc1</smiles>

2f

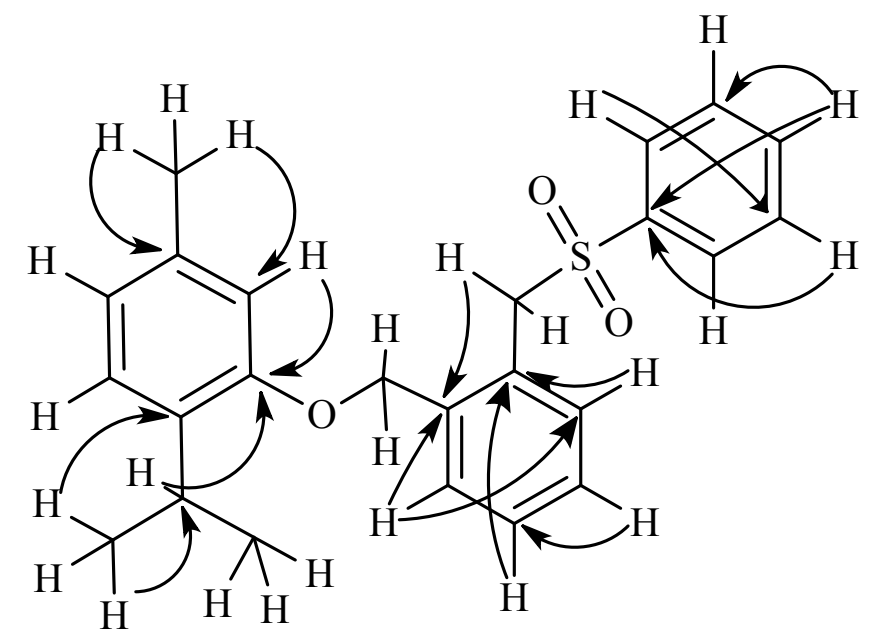

${ }^{1} \mathrm{H}$ NMR (400 MHz, $1.14\left(6 \mathrm{H}, \mathrm{d}, \mathrm{CH}_{3}-8\right.$ and $\left.\mathrm{CH}_{3}-9\right) ; 2.34(3 \mathrm{H}, \mathrm{s}$, DMSO- $d_{6}$ ) $\mathrm{CH}_{2}-1$ '”); $4.93\left(2 \mathrm{H}, \mathrm{s}, \mathrm{CH}_{2}-1^{\prime}\right) ; 6.69(1 \mathrm{H}, \mathrm{s}, \mathrm{H}-$ 6); $6.79\left(1 \mathrm{H}, \mathrm{d}, J_{4,3} 7.78, \mathrm{H}-4\right) ; 7.05\left(1 \mathrm{H}, \mathrm{d}, J_{6},{ }^{\prime \prime}\right.$, 8.24, H-6"); $7.10\left(1 \mathrm{H}, \mathrm{d}, J_{3,4} 7.78, \mathrm{H}-3\right) ; 7.24$

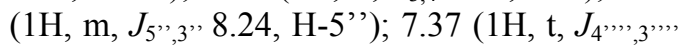

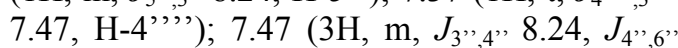

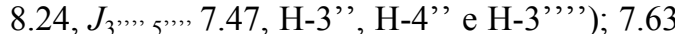
$\left(1 \mathrm{H}, \mathrm{m}, J_{5},{ }^{\prime}, 6^{\prime \prime}, 7.47, \mathrm{H}-5^{\prime},{ }^{\prime},\right) ; 7.68(2 \mathrm{H}, \mathrm{m}$,

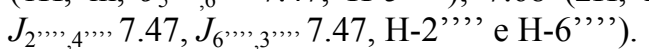

${ }^{13} \mathrm{C}$ NMR (100 MHz， 21.36 (C-10); 22.87 (C-8, C-9); 26.41 (C-7); DMSO- $\left.d_{6}\right)$ 59.58 (C-1'); 68.17 (C-1'"'); 112.78 (C-6); 121.78 (C-4); 126.03 (C-3); 126.56 (C-2"'); 128.16 (C-4"'); 128.62 (C-3"'"); 129.56 (C-5"); 132.56 (C-5"',); $133.90(\mathrm{C}-2) ; 136.46(\mathrm{C}-5)$; 137.59 (C-1'); 138.80 (C-1'”'); 155.51 (C-1).

Infra-red (cm $\left.{ }^{-1}\right) \quad 2926\left(\mathrm{C}-\mathrm{H}\right.$ in $\mathrm{CH}_{2}-1, \cdots, \mathrm{CH}_{2}-1$, CH-7, $\mathrm{CH}_{3}-8$, $\mathrm{CH}_{3}-9$ and $\left.\mathrm{CH}_{3}-10\right) ; 1315$ and $1150\left(\mathrm{SO}_{2}\right.$ in $\mathrm{C}-$ $1, "$, and $\mathrm{CH}_{2}-1$ "'); 1254 and 1034 (ether in $\mathrm{CH}_{2}-1$ ' and $\left.\mathrm{C}-1\right)$.

Mass spectrometry HRESIMS $[\mathrm{M}+\mathrm{Na}]^{+}$Found: 417.1494 Calc. for $\mathrm{C}_{24} \mathrm{H}_{26} \mathrm{NaO}_{3} \mathrm{~S}^{+}: 417.1500$

Table S6. Spectroscopic data for compound $\mathbf{2 f}$

Figure S33. Compound $2 \mathbf{f}$ and main $\mathrm{HMBC}$ correlation $J$ in $\mathrm{Hz}$. 

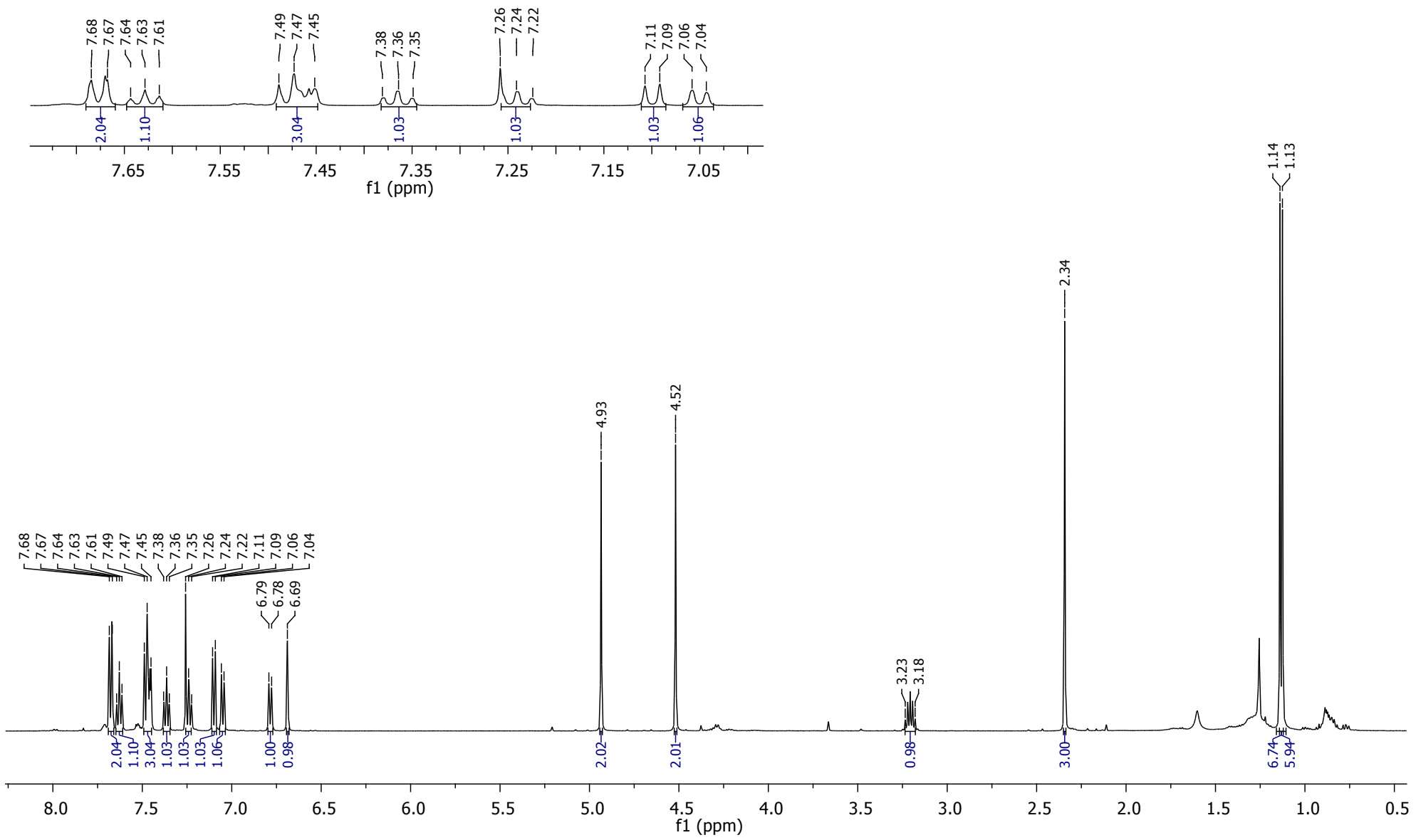

Figure S34. ${ }^{1} \mathrm{H}$ NMR spectrum (400 MHz, DMSO- $d_{6}$ ) of compound (1-isopropyl-4-methyl-2-((2-((phenylsulfonyl)methyl)benzyl)oxy)benzene) (2f). 


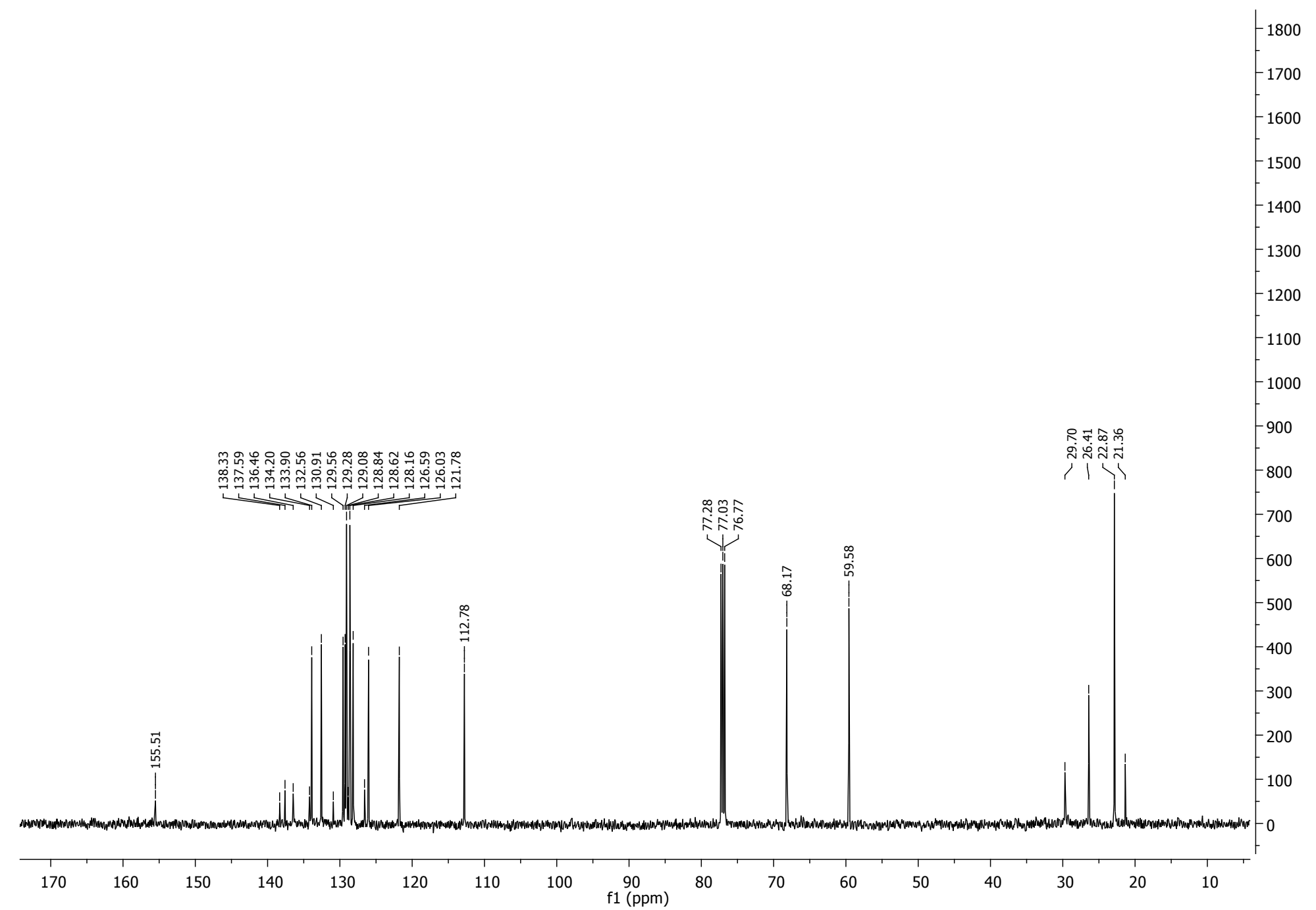

Figure S35. ${ }^{13} \mathrm{C}$ NMR spectrum (100 MHz, DMSO- $\left.d_{6}\right)$ of compound (1-isopropyl-4-methyl-2-((2-((phenylsulfonyl)methyl)benzyl)oxy)benzene) (2f). 


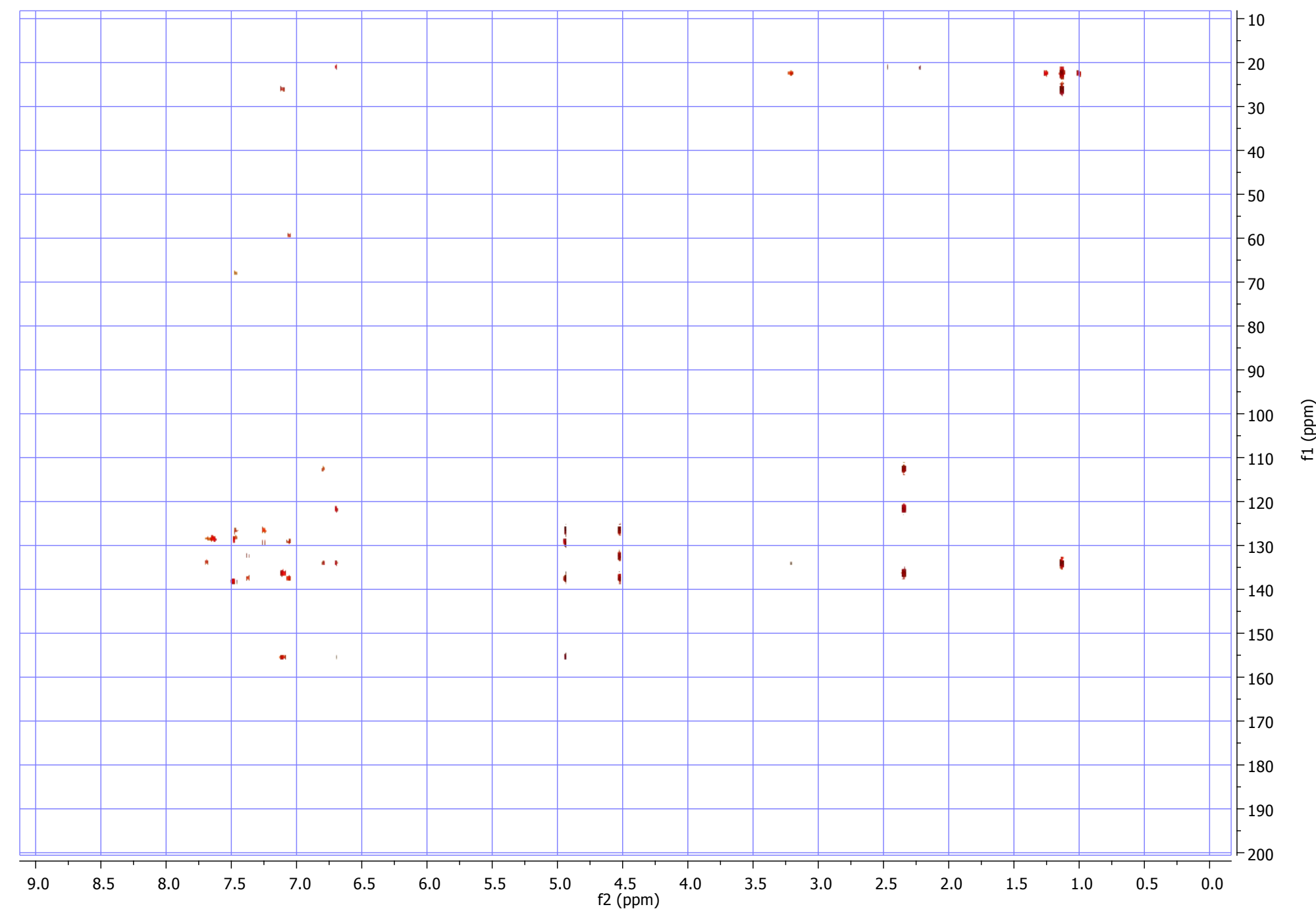

Figure S36 - HMBC spectrum (400 MHz, DMSO- $d_{6}$ ) of the compound (1-isopropyl-4-methyl-2-((2-((phenylsulfonyl)methyl)benzyl)oxy)benzene) (2f). 


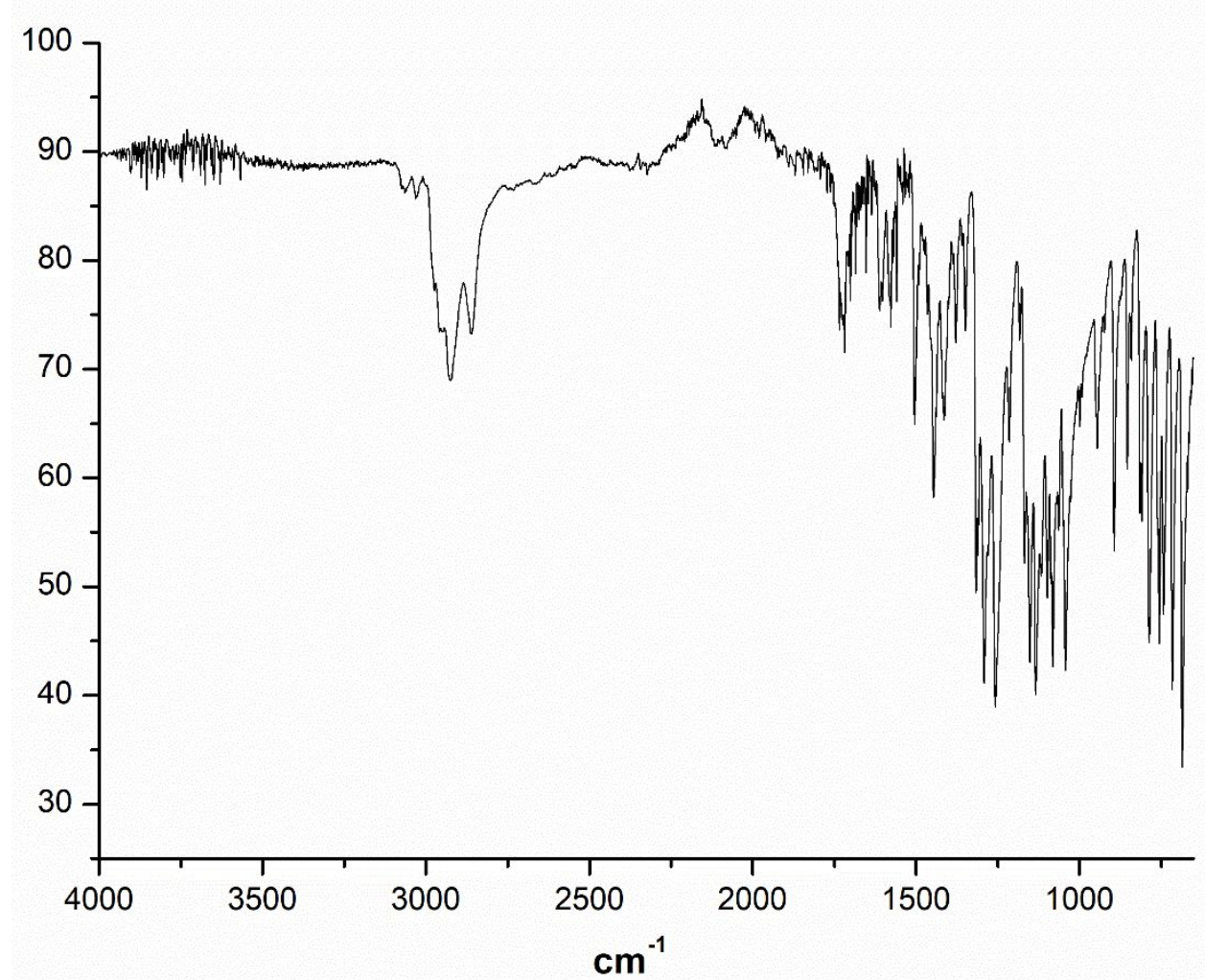

Figure S37. IR spectrum of compound 2 f. 


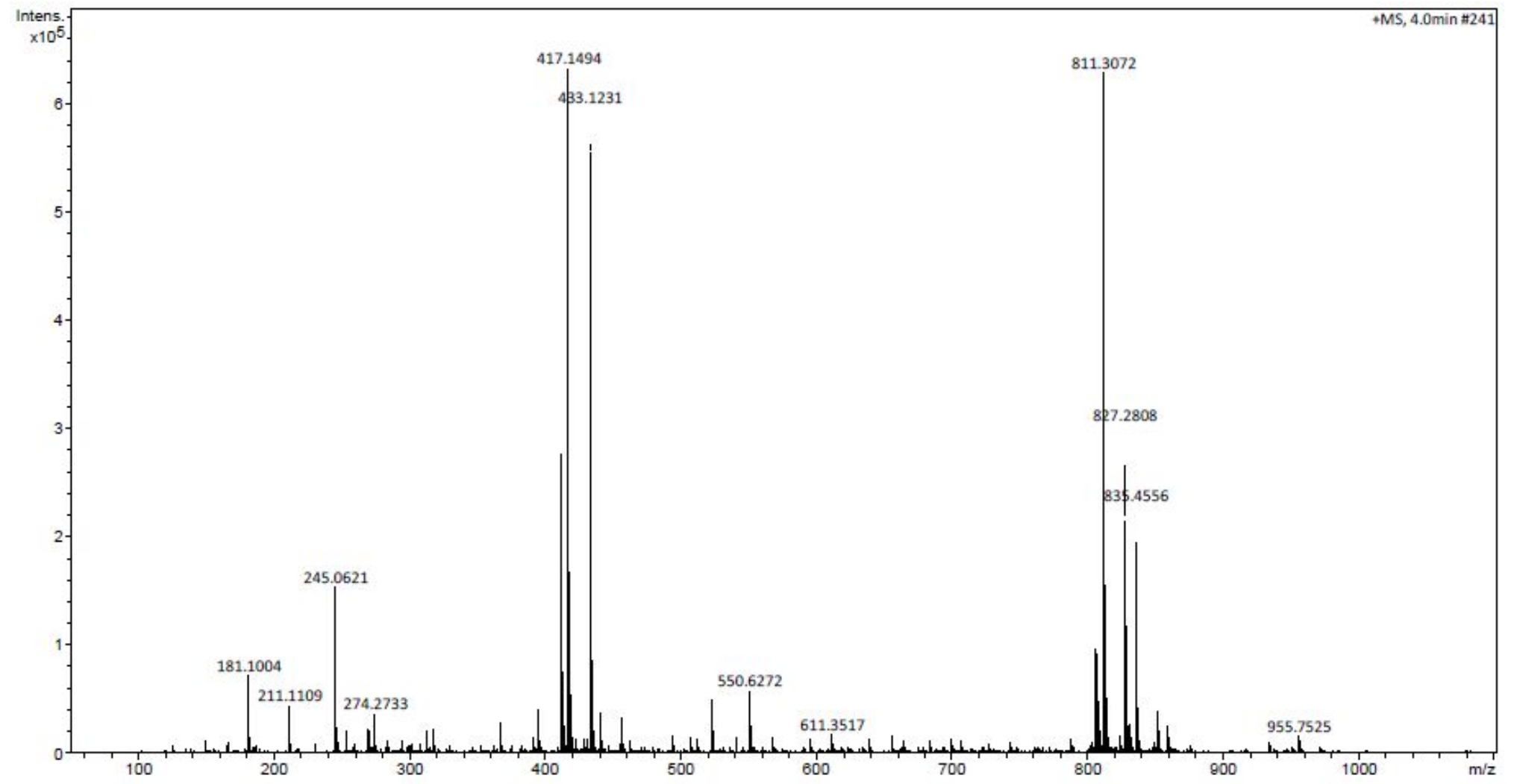

Figure S38. HRMS spectrum of compound $\mathbf{2 f}$. 

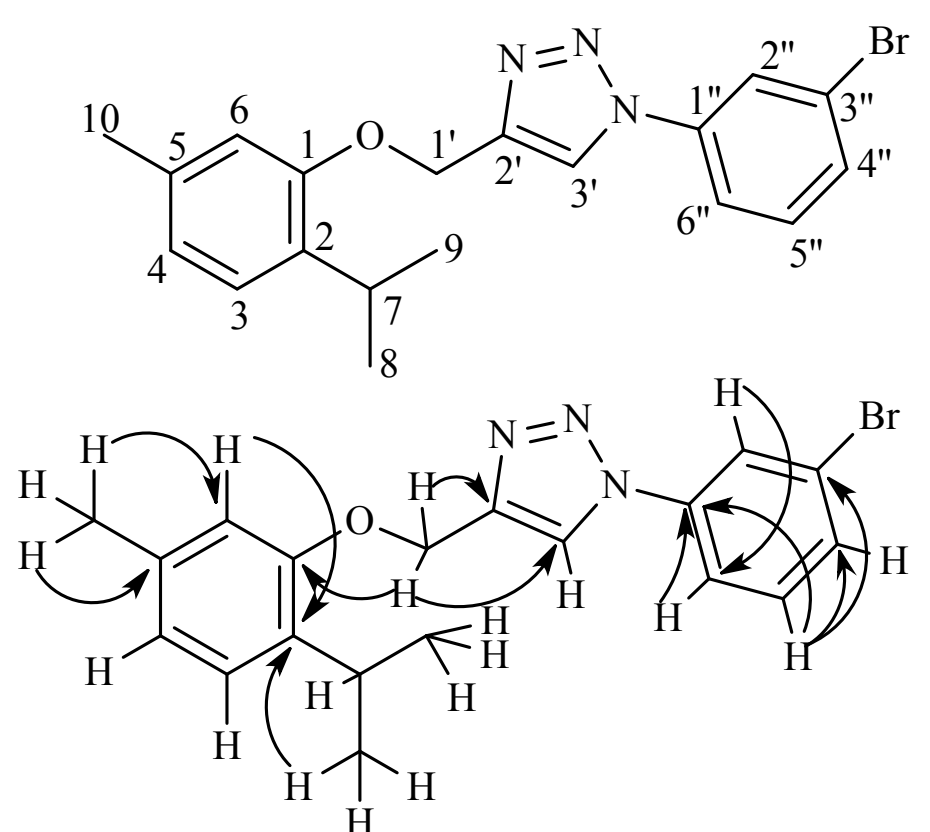

Figure S39. Compound 3d and main HMBC correlation $J$ in $\mathrm{Hz}$.
${ }^{1}$ H NMR (400 MHz, $1.05\left(6 \mathrm{H}, \mathrm{d}, \mathrm{CH}_{3}-8\right.$ and $\left.\mathrm{CH}_{3}-9\right) ; 2.24(3 \mathrm{H}, \mathrm{s}$, DMSO- $d_{6}$ )

$\left.\mathrm{CH}_{3}-10\right) ; 3.10-3.18(1 \mathrm{H}, \mathrm{m}, \mathrm{H}-7) ; 5.16(2 \mathrm{H}, \mathrm{s}$, $\left.\mathrm{CH}_{2}-1^{\prime}\right) ; 6.70\left(1 \mathrm{H}, \mathrm{d}, J_{4,3} 7.82, \mathrm{H}-4\right) ; 6.95(1 \mathrm{H}, \mathrm{s}$, H-6); $7.02\left(1 \mathrm{H}, \mathrm{d}, J_{3,4} 7.82, \mathrm{H}-3\right) ; 7.52(1 \mathrm{H}, \mathrm{t}$, $\left.J_{5},{ }, 48.21, \mathrm{H}-5, '\right) ; 7.66\left(1 \mathrm{H}, \mathrm{dd}, J_{4},{ }^{\prime},{ }^{\prime}, 8.21, \mathrm{H}-\right.$ 4 ', $) ; 7.92\left(1 \mathrm{H}, \mathrm{dd}, J_{6},{ }_{5}, 8.21, \mathrm{H}-6, "\right) ; 8.13(1 \mathrm{H}$, t, H-2'"); 8.91 (1H, s, H-triazole).

${ }^{13}$ C NMR (100 MHz, 21.35 (C-10); 23.09 (C-8, C-9); 26.33 (C-7); DMSO-d D $^{2}$ $61.71\left(\mathrm{C}-1^{\prime}\right) ; 113.57(\mathrm{C}-6) ; 119.56\left(\mathrm{C}-4^{\prime}\right)$ ); 122.06 (C-4); 122.85 (C-2’'); 123.07 (C-3'); 123.13 (C-1'’);126.11 (C-3); 131.95 (C-6'); 132.30 (C-5'); 133.90 (C-2); 136.40 (C-5); 138.02 (C-3'”); 144.88 (C-2'); 155.39 (C-1).

Infra-red $\left(\mathrm{cm}^{-1}\right)$

3049 (C-H in $\mathrm{CH}_{2}-1$ ', $\mathrm{CH}-7, \mathrm{CH}_{3}-8, \mathrm{CH}_{3}-9$, $\mathrm{CH}_{3}-10$ ); 1250 and 1016 (ether in $\mathrm{CH}_{2}-1^{\prime}$ and $\mathrm{C}$ 1); 1045 (C-Br in C-3"').

Mass spectrometry

HRESIMS [M + Na] $]^{+}$Found: 408.0693 Calc. for $\mathrm{C}_{19} \mathrm{H}_{20} \mathrm{BrN}_{3} \mathrm{NaO}^{+}: 408.0687$

Table S7. Spectroscopic data for compound 3d. 

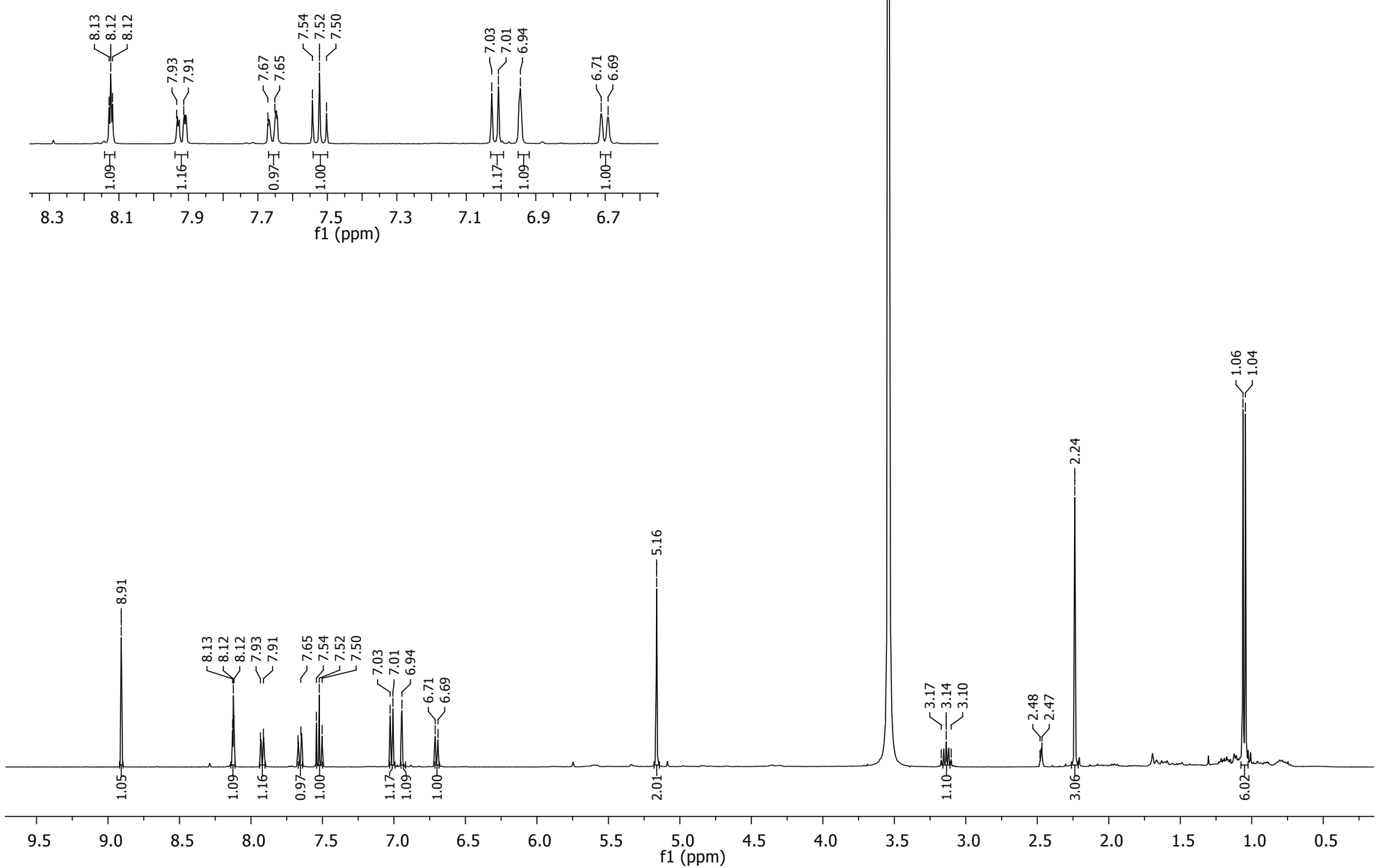

Figure S40. ${ }^{1} \mathrm{H}$ NMR spectrum (400 MHz, DMSO- $d_{6}$ ) of compound (1-(3-bromophenyl)-4-((2-isopropyl-5-methylphenoxy)methyl)-1H-1,2,3-triazole) (3d). 


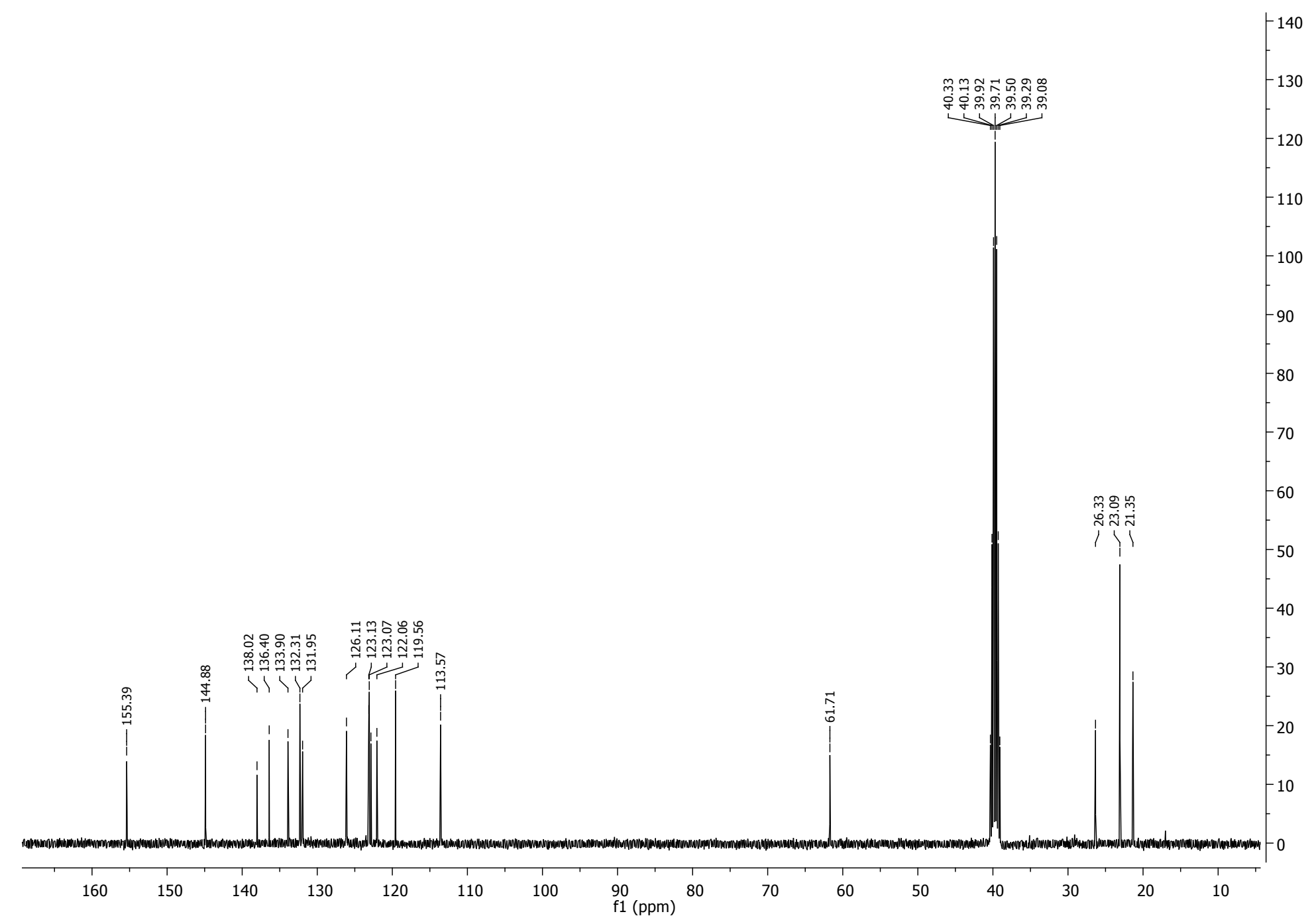

Figure S41. ${ }^{13} \mathrm{C}$ NMR spectrum (100 MHz, DMSO- $\left.d_{6}\right)$ of compound (1-(3-bromophenyl)-4-((2-isopropyl-5-methylphenoxy)methyl)-1 $H$-1,2,3-triazole) (3d). 


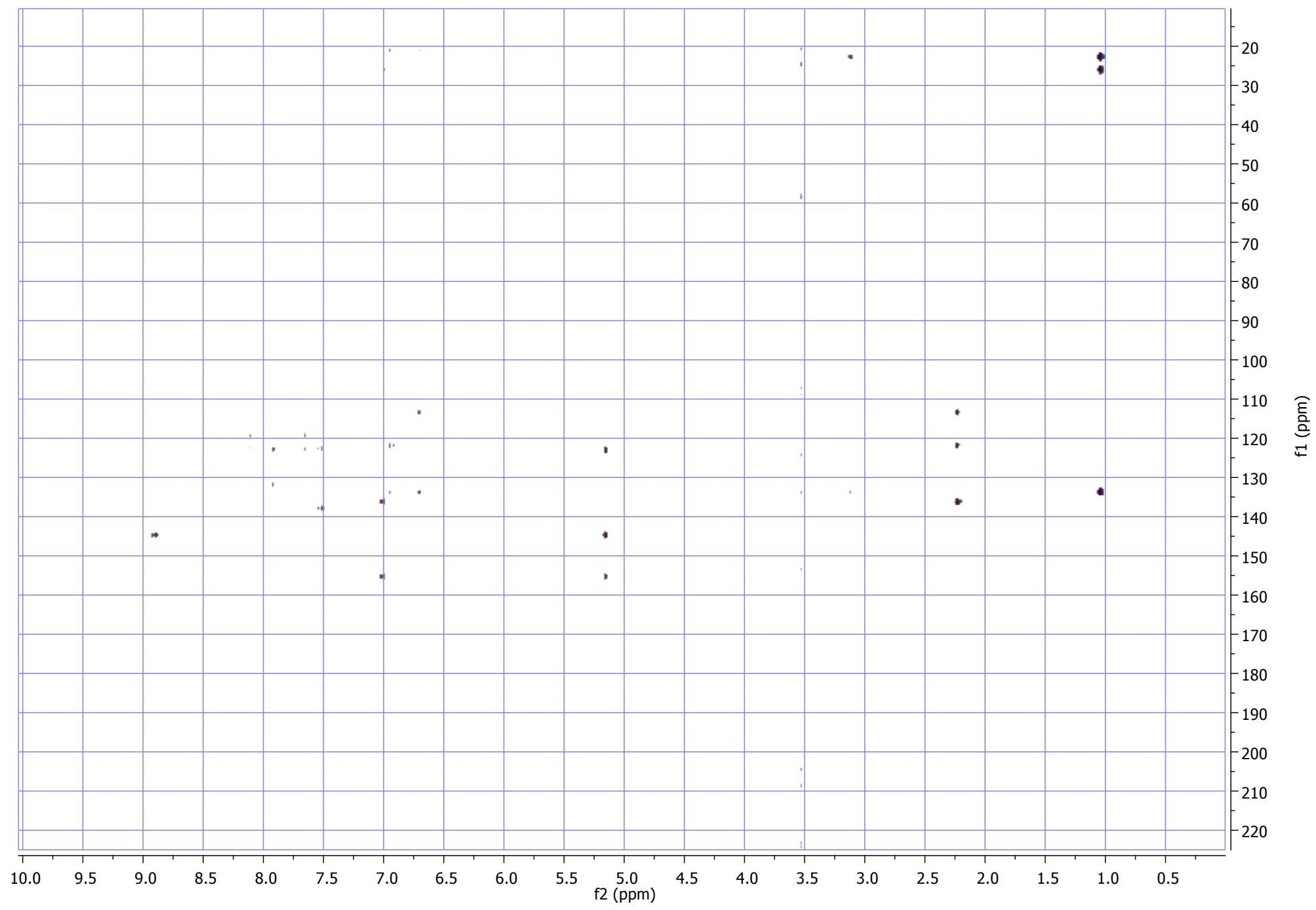

Figure S42 - HMBC spectrum (400 MHz, DMSO- $d_{6}$ ) of the compound (1-(3-bromophenyl)-4-((2-isopropyl-5-methylphenoxy)methyl)-1 $H$-1,2,3-triazole) (3d). 


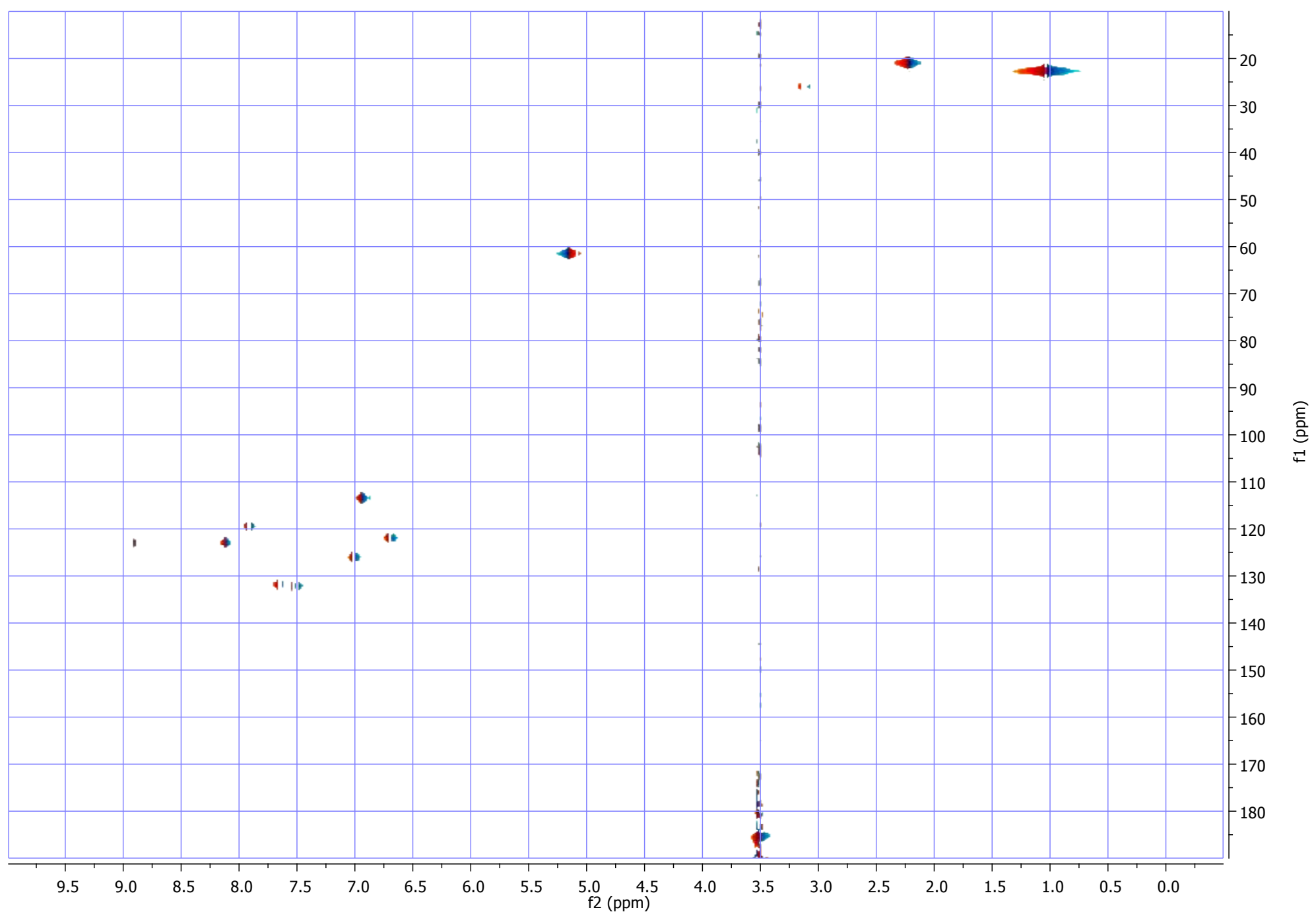

Figure S43 - HSQC spectrum (400 MHz, DMSO- $d_{6}$ ) of the compound (1-(3-bromophenyl)-4-((2-isopropyl-5-methylphenoxy)methyl)-1 $H$-1,2,3-triazole) (3d). 


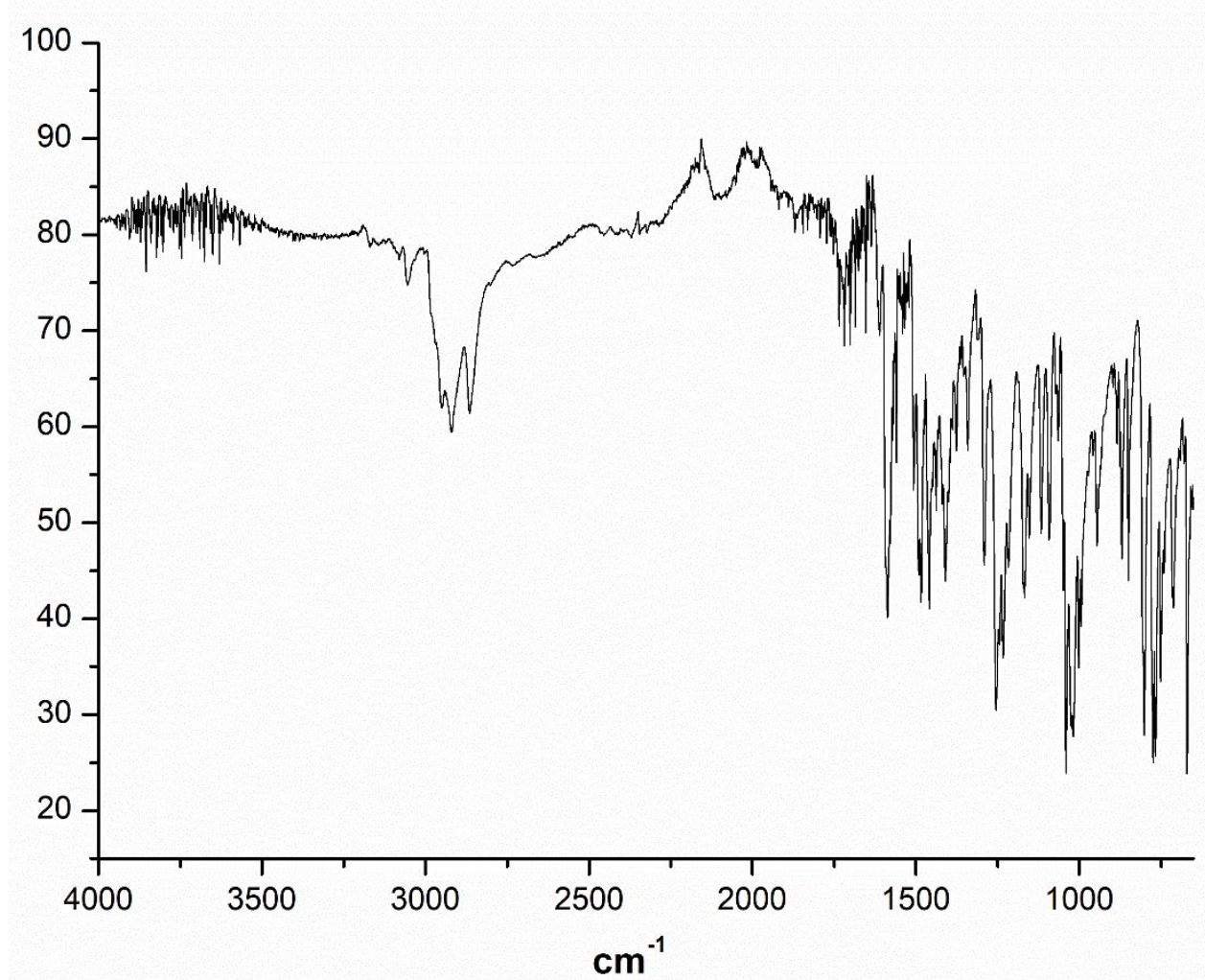

Figure S44. IR spectrum of compound 3d. 


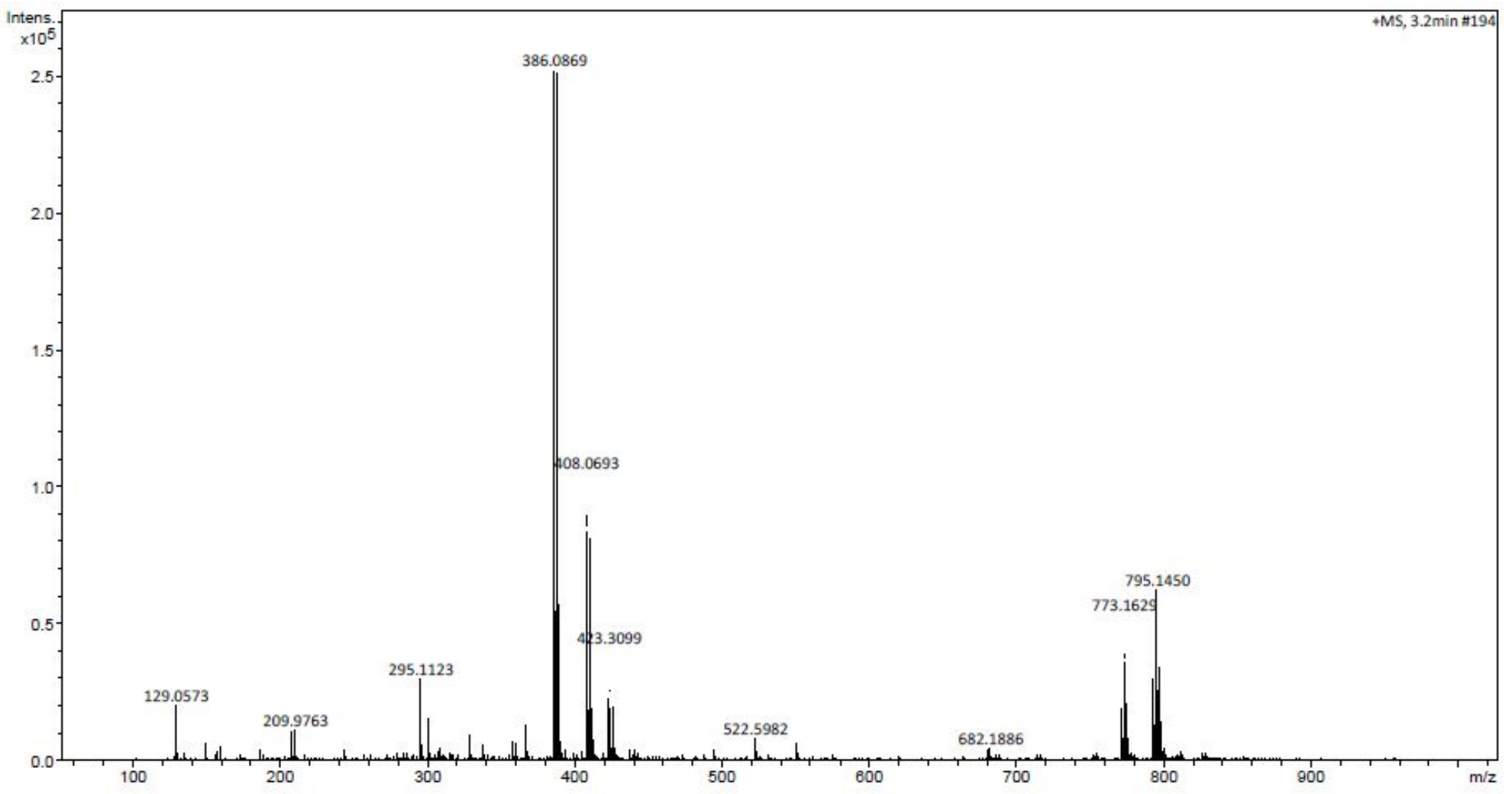

Figure S45. HRMS spectrum of compound 3d. 


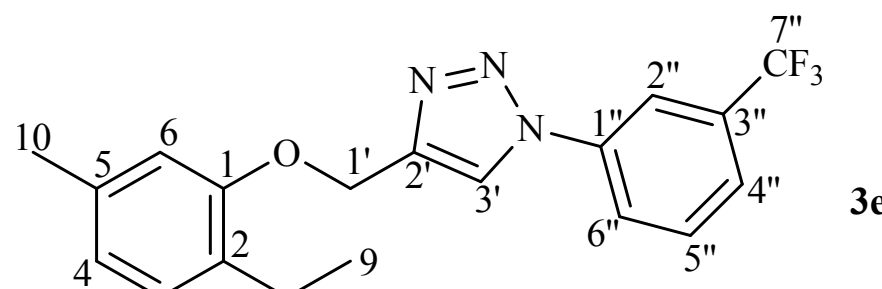

$3 \mathbf{e}$

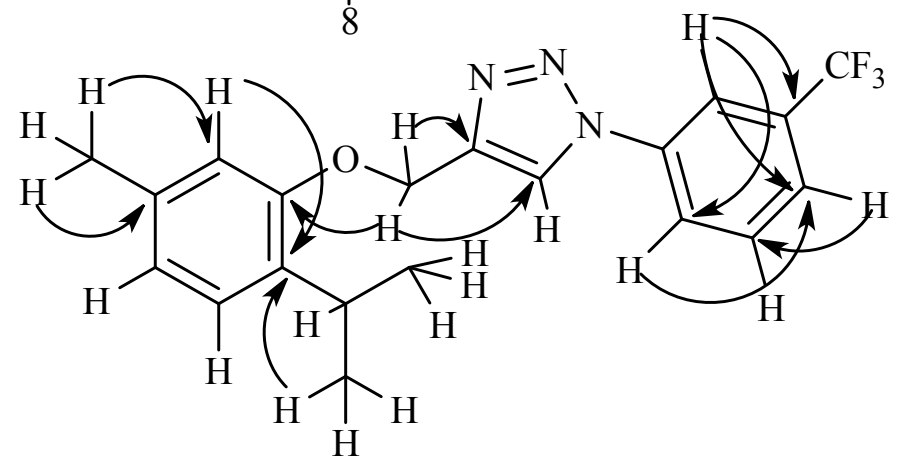

Figure S46. Compound 3e and main $\mathrm{HMBC}$ correlation $J$ in $\mathrm{Hz}$.
${ }^{1} \mathbf{H}$ NMR (400 MHz, $1.06\left(6 \mathrm{H}, \mathrm{d}, \mathrm{CH}_{3}-8\right.$ and $\left.\mathrm{CH}_{3}-9\right) ; 2.24(3 \mathrm{H}, \mathrm{s}$ DMSO- $\left.d_{6}\right)$

$\left.\mathrm{CH}_{3}-10\right) ; 3.13-3.20(1 \mathrm{H}, \mathrm{m}, \mathrm{H}-7) ; 5.19(2 \mathrm{H}, \mathrm{s}$ $\left.\mathrm{CH}_{2}-1{ }^{\prime}\right) ; 6.70\left(1 \mathrm{H}, \mathrm{d}, J_{4,3} 7.82, \mathrm{H}-4\right) ; 6.96(1 \mathrm{H}, \mathrm{s}$, H-6); $7.10\left(1 \mathrm{H}, \mathrm{d}, J_{3,4} 7.82, \mathrm{H}-3\right) ; 7.83-7.77(2 \mathrm{H}$, m, $J_{5},, 6,8.21, \mathrm{H}-5$ ' and H-6, $) ; 8.27-8.20(2 \mathrm{H}$ m, $J_{4},{ }^{\prime}, 5,8.21, \mathrm{H}-2$ "' and H-4"'); 9.05 (1H, s, Htriazole).

${ }^{13}$ C NMR (100 MHz, 21.34 (C-10); 23.07 (C-8, C-9); 26.33 (C-7); DMSO-d D $^{2}$ 61.79 (C-1'); 113.56 (C-6); 117.19 (C-2'”); 122.01 (C-4); 123.28 (C-3'); 124.44 (C-4'”); 125.66 (C-6’'); 126.06 (C-3); 131.71 (C-5'); 133.88 (C-2); 136.35 (C-5); 137.43 (C-3''); 145.01 (C-2'); 155.44 (C-1).

Infra-red $\left(\mathrm{cm}^{-1}\right)$

2968 (C-H in $\mathrm{CH}_{2}-1$ ', $\mathrm{CH}-7, \mathrm{CH}_{3}-8, \mathrm{CH}_{3}-9$, $\mathrm{CH}_{3}-10$ ); 1247 and 1034 (ether in $\mathrm{CH}_{2}-1^{\prime}$ and $\mathrm{C}$ 1); $1126\left(\mathrm{C}^{-\mathrm{CF}_{3}}\right.$ in $\mathrm{C}-3$ '').

Mass spectrometry HRESIMS $[\mathrm{M}+\mathrm{Na}]^{+}$Found: $398.1461 \mathrm{Calc}$. for $\mathrm{C}_{20} \mathrm{H}_{20} \mathrm{~F}_{3} \mathrm{~N}_{3} \mathrm{NaO}^{+}$: 398.1456 .

Table S8. Spectroscopic data for compound 3e. 

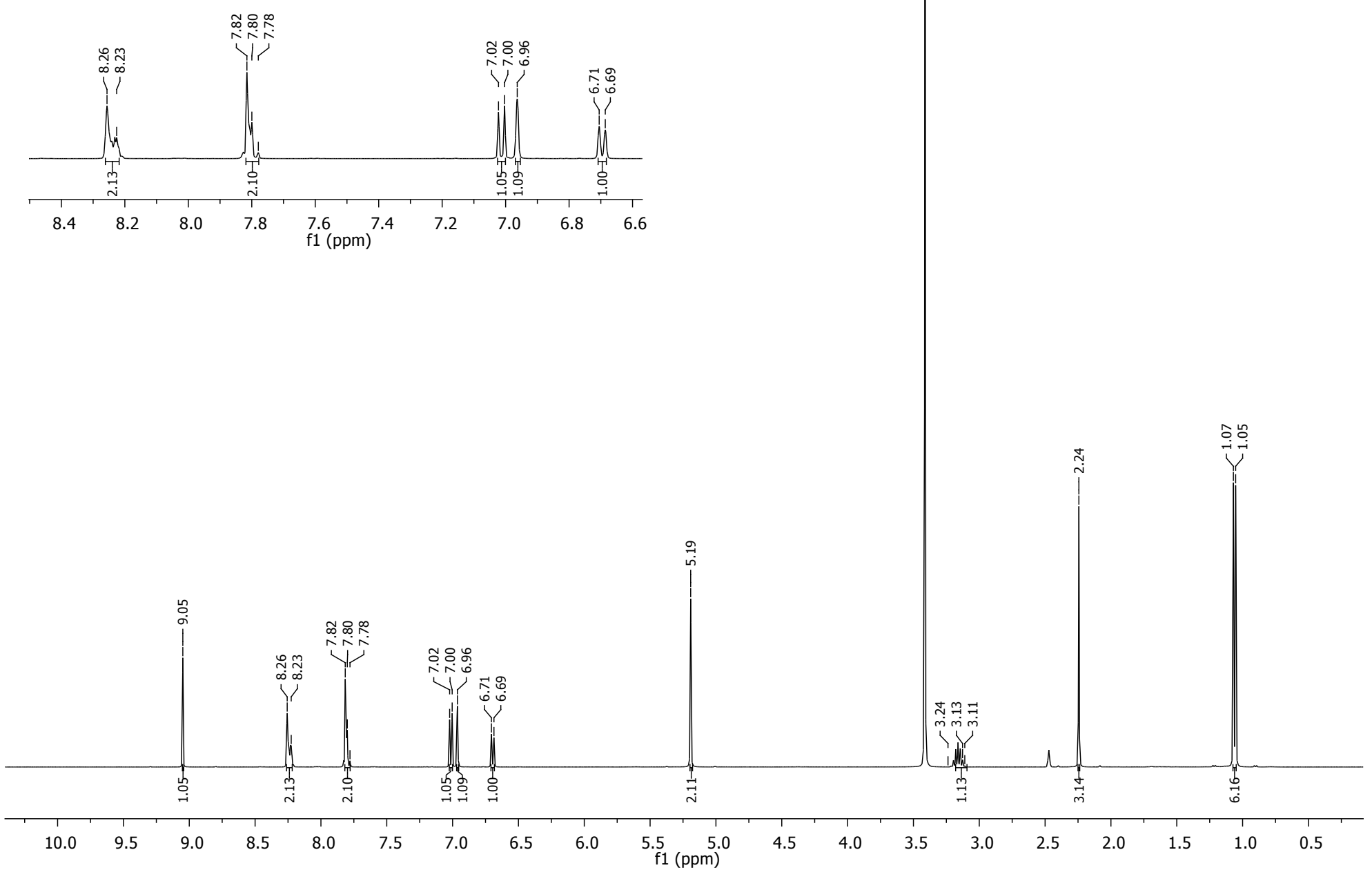

Figure S47. ${ }^{1} \mathrm{H}$ NMR spectrum (400 MHz, DMSO- $d_{6}$ ) of compound (4-((2-isopropyl-5-methylphenoxy)methyl)-1-(3-(trifluoromethyl)phenyl)-1 $H$-1,2,3-triazole) $(3 \mathbf{e})$. 


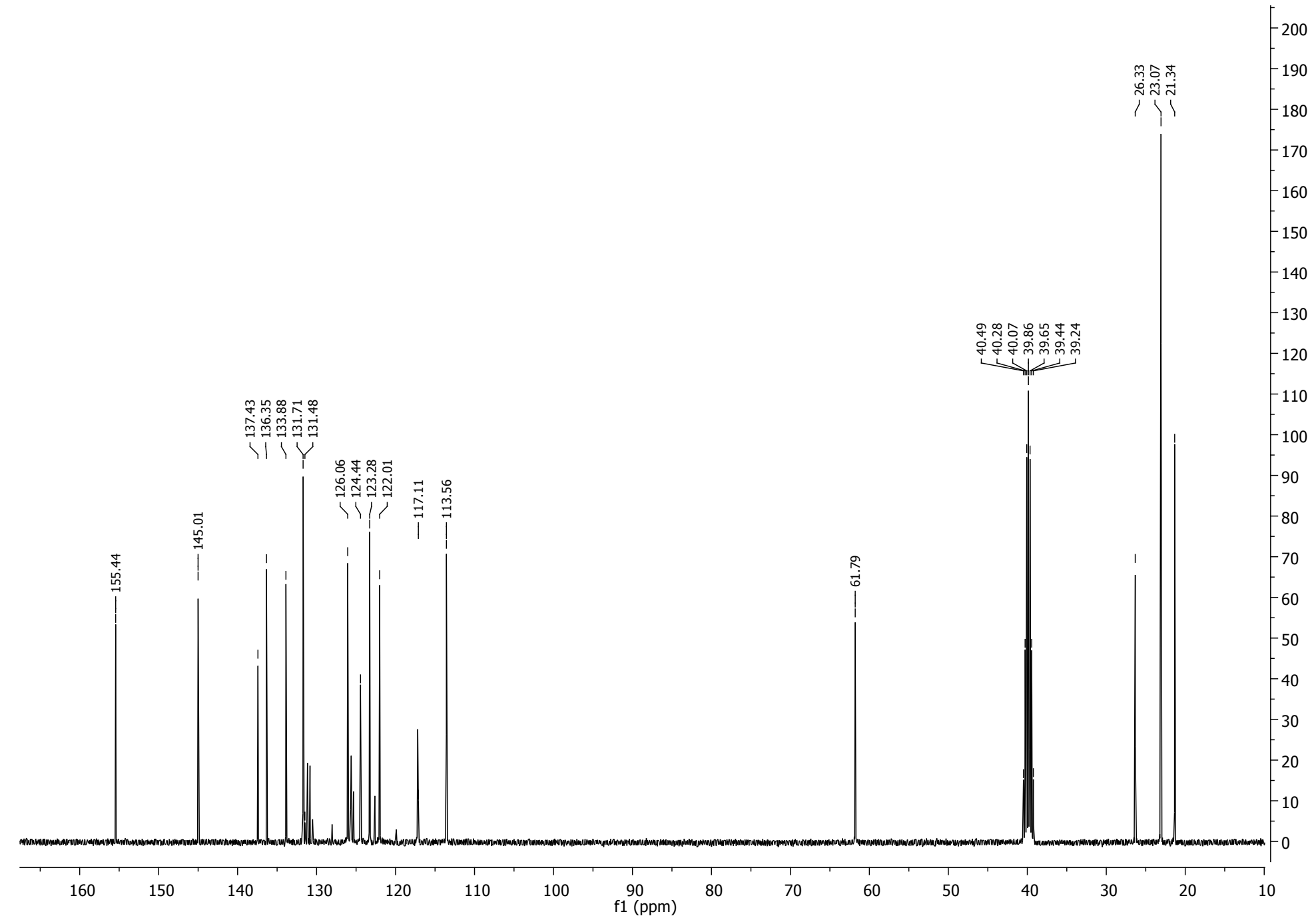

Figure S48. ${ }^{13} \mathrm{C}$ NMR spectrum (100 MHz, DMSO- $d_{6}$ ) of compound (4-((2-isopropyl-5-methylphenoxy)methyl)-1-(3-(trifluoromethyl)phenyl)-1H-1,2,3-triazole) (3e). 


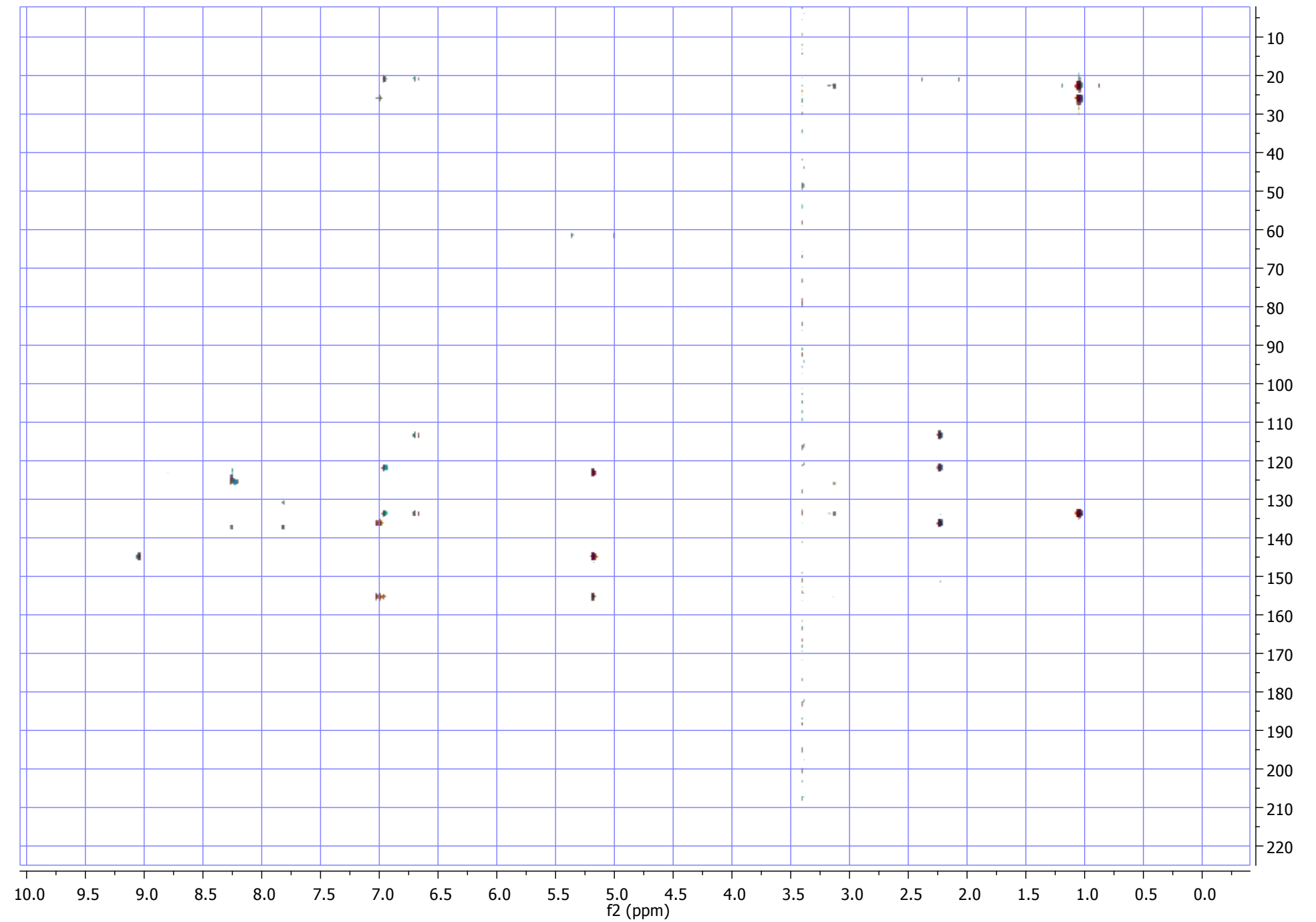

Figure S49 - HMBC spectrum (400 MHz, DMSO- $d_{6}$ ) of the compound (4-((2-isopropyl-5-methylphenoxy)methyl)-1-(3-(trifluoromethyl)phenyl)-1H-1,2,3triazole) (3e). 


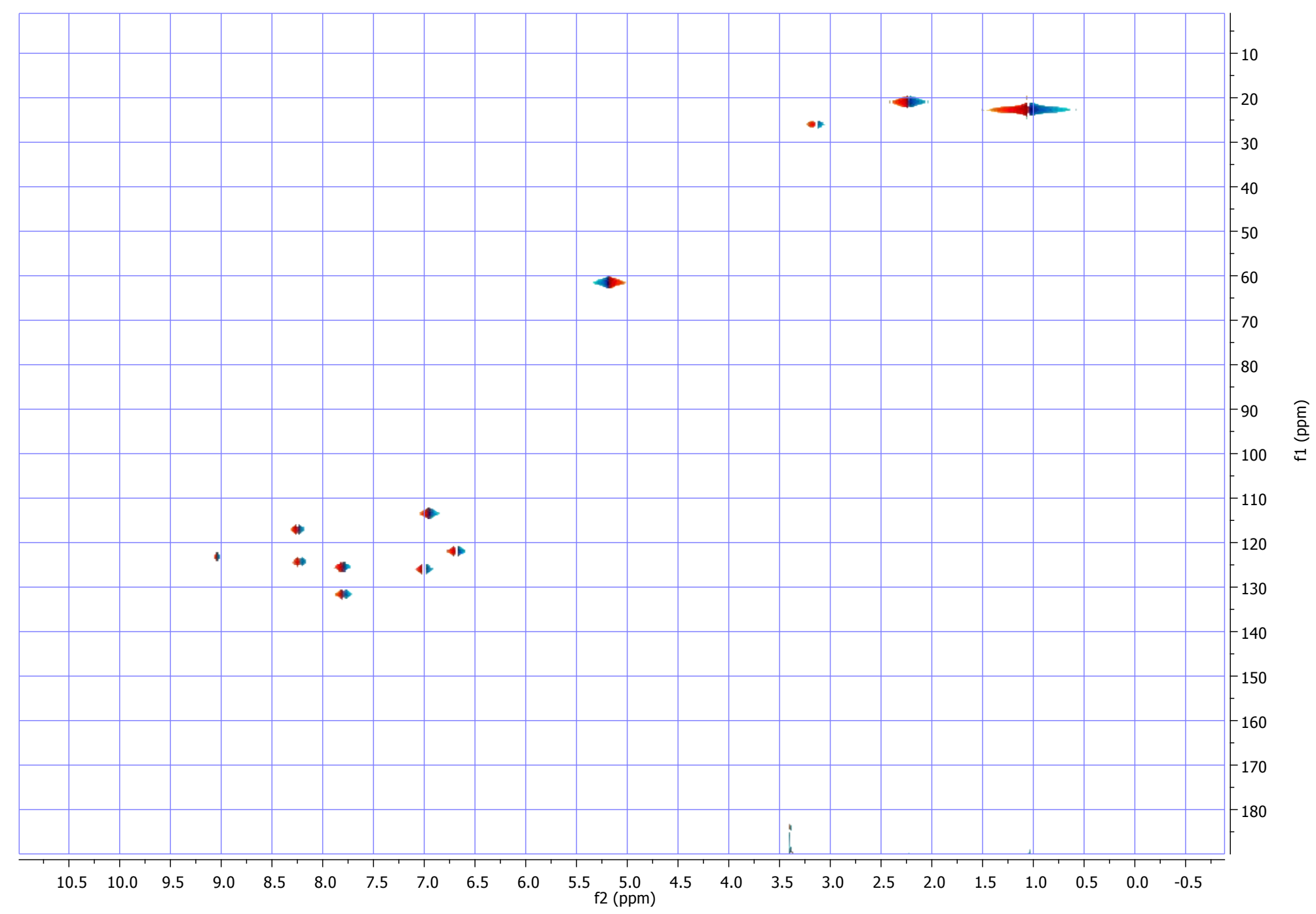

Figure S50 - HSQC spectrum (400 MHz, DMSO- $d_{6}$ ) of the compound (4-((2-isopropyl-5-methylphenoxy)methyl)-1-(3-(trifluoromethyl)phenyl)-1 $H$-1,2,3triazole) (3e). 


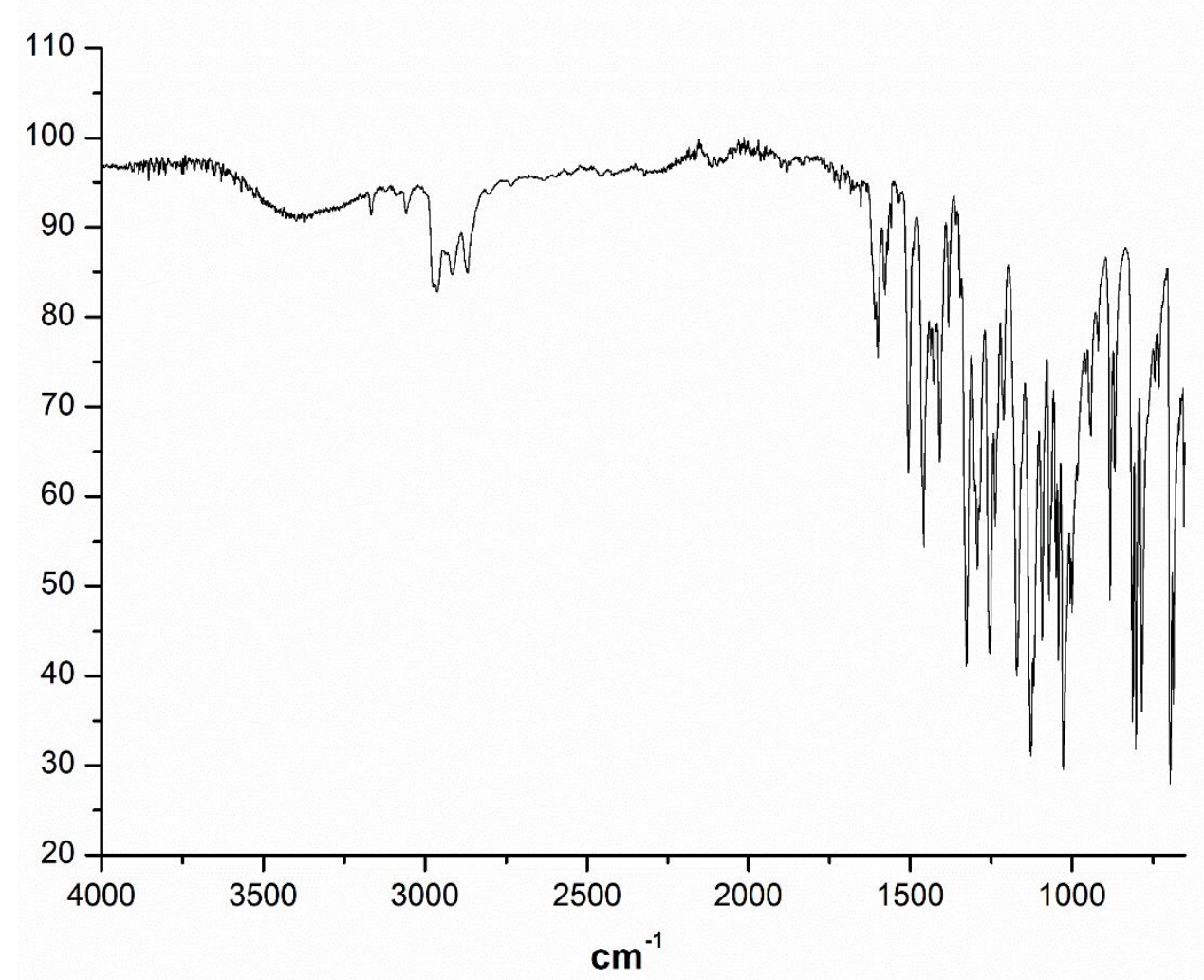

Figure S51. IR spectrum of compound $3 \mathbf{e}$. 


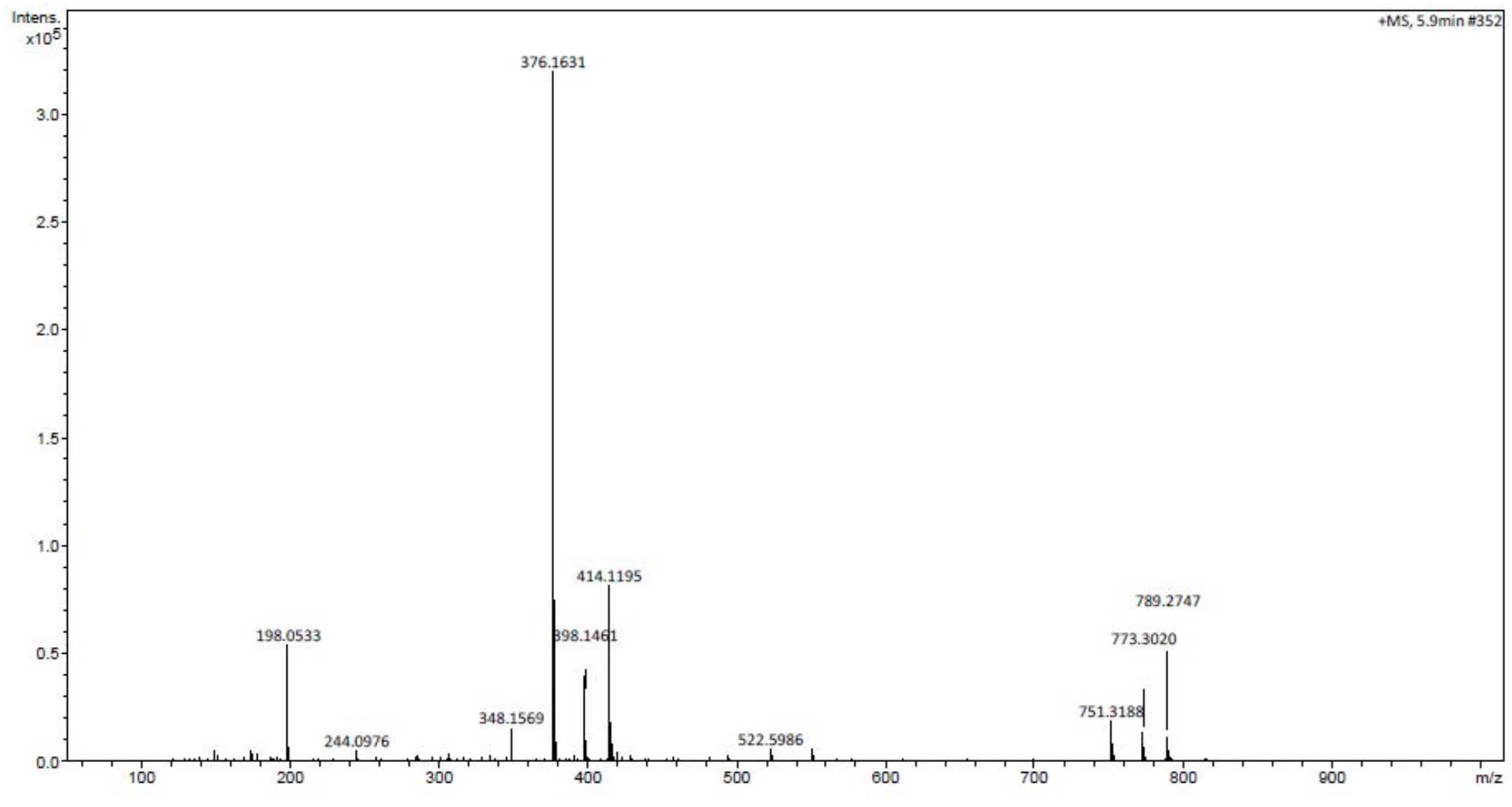

Figure S52. HRMS spectrum of compound $3 \mathbf{e}$. 

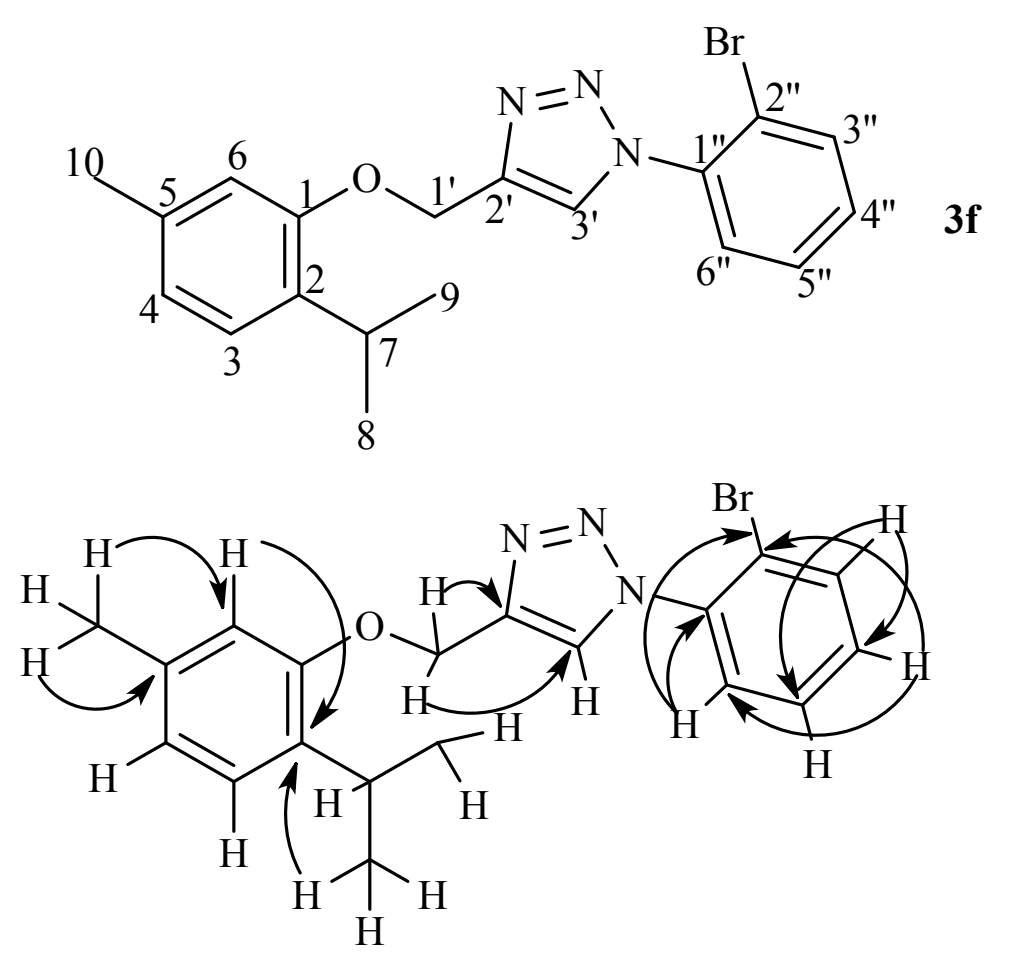

Product Aspect

Pale yellow solid

${ }^{1}$ H NMR (400 MHz, $1.08\left(6 \mathrm{H}, \mathrm{d}, \mathrm{CH}_{3}-8\right.$ and $\left.\mathrm{CH}_{3}-9\right) ; 2.25(3 \mathrm{H}, \mathrm{s}$, DMSO- $d_{6}$ )

CH -10$) ; 3.13-3.20(1 \mathrm{H}, \mathrm{m}, \mathrm{H}-7) ; 5.21(2 \mathrm{H}, \mathrm{s}$ $\mathrm{CH}_{2}-1$ '); $6.71\left(1 \mathrm{H}, \mathrm{d}, J_{4,3} 7.82, \mathrm{H}-4\right) ; 6.97(1 \mathrm{H}, \mathrm{s}$ H-6); $7.03\left(1 \mathrm{H}, \mathrm{d}, J_{3,4} 7.82, \mathrm{H}-3\right) ; 7.52(1 \mathrm{H}, \mathrm{td}$, $\left.J_{6},, 5,7.82, \mathrm{H}-6,\right) ; 7.59\left(1 \mathrm{H}, \mathrm{td}, J_{5}, 4,4.82, \mathrm{H}-\right.$ 5,"); $7.63\left(1 \mathrm{H}, \mathrm{dd}, J_{4},{ }^{\prime}, 7.82, \mathrm{H}-4, "\right) ; 7.88(1 \mathrm{H}$, dd, $J_{3}, 5^{\prime}, 7.82, \mathrm{H}-3$ '”); 8.58 (1H, s, H-triazole).

${ }^{13}$ C NMR (100 MHz, 21.38 (C-10); 23.10 (C-8, C-9); 26.46 (C-7); DMSO-d D) $^{-(2)}$ $61.87\left(\mathrm{C}-1^{\prime}\right) ; 113.80(\mathrm{C}-6) ; 119.15\left(\mathrm{C}-1^{\prime}\right)$; 122.02 (C-4); 126.11 (C-3); 126.83 (C-3'); 129.08 (C-4'); 129.41 (C-5' '); 132.39 (C-6'); 133.96 (C-2); 134.06 (C-3'); 136.35 (C-2''); 136.54 (C-5); 143.70 (C-2’); 155.49 (C-1).

Infra-red $\left(\mathrm{cm}^{-1}\right)$

2956 (C-H in $\mathrm{CH}_{2}-1$ ', $\mathrm{CH}-7, \mathrm{CH}_{3}-8, \mathrm{CH}_{3}-9$, $\mathrm{CH}_{3}-10$ ); 1247 and 1095 (ether in $\mathrm{CH}_{2}-1$ ' and $\mathrm{C}$ 1); 1035 (C-Br in C-2'’).

Mass spectrometry HRESIMS $[\mathrm{M}+\mathrm{H}]^{+}$Found: 386.0873 Calc. for $\mathrm{C}_{19} \mathrm{H}_{21} \mathrm{BrN}_{3} \mathrm{O}^{+}: 386.0868$.

Table S9. Spectroscopic data for compound 3f.

Figure S53. Compound 3f and main HMBC correlation $J$ in $\mathrm{Hz}$. 

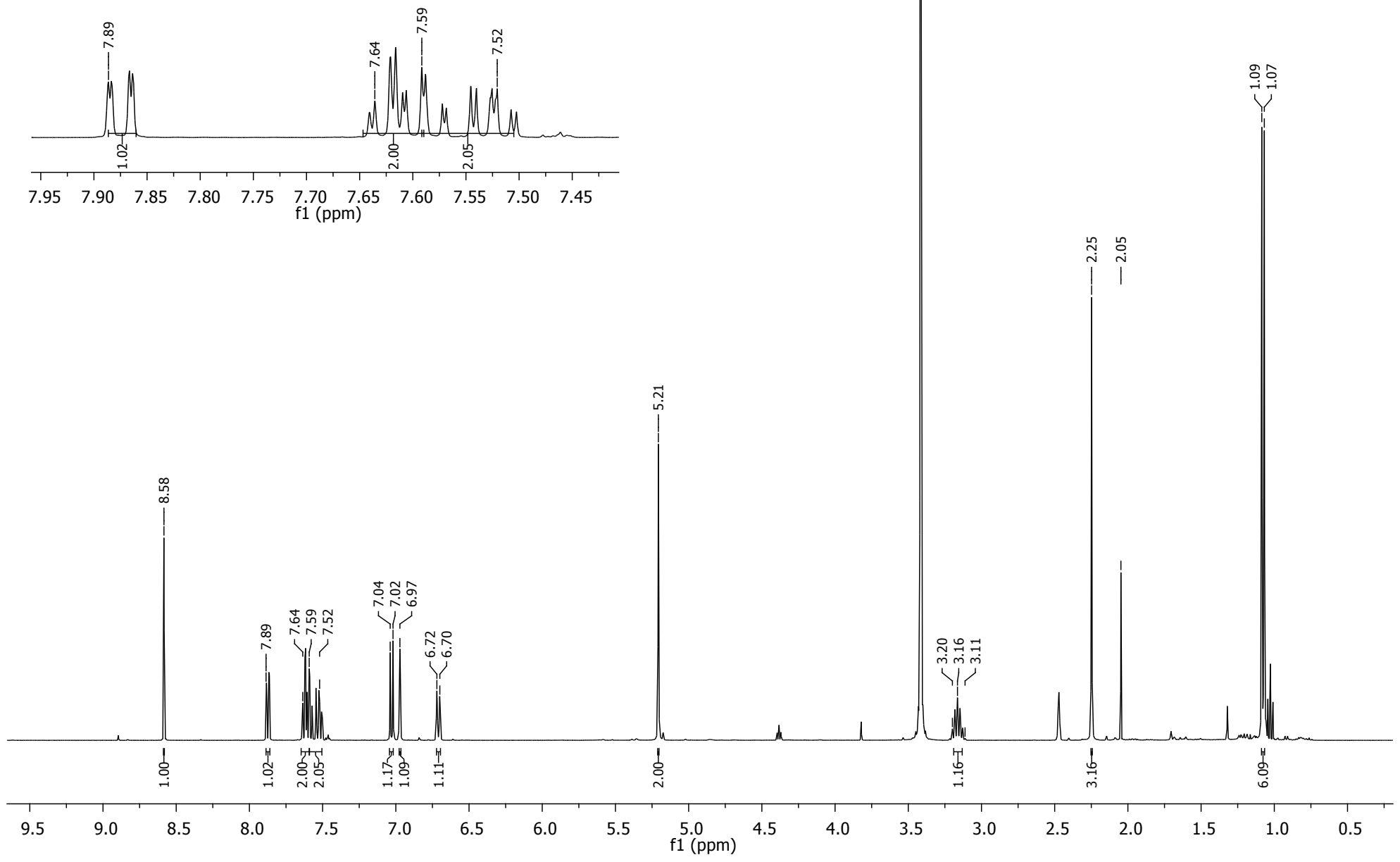

Figure S54. ${ }^{1} \mathrm{H}$ NMR spectrum (400 MHz, DMSO- $d_{6}$ ) of compound (1-(2-bromophenyl)-4-((2-isopropyl-5-methylphenoxy)methyl)-1H-1,2,3-triazole) (3f). 


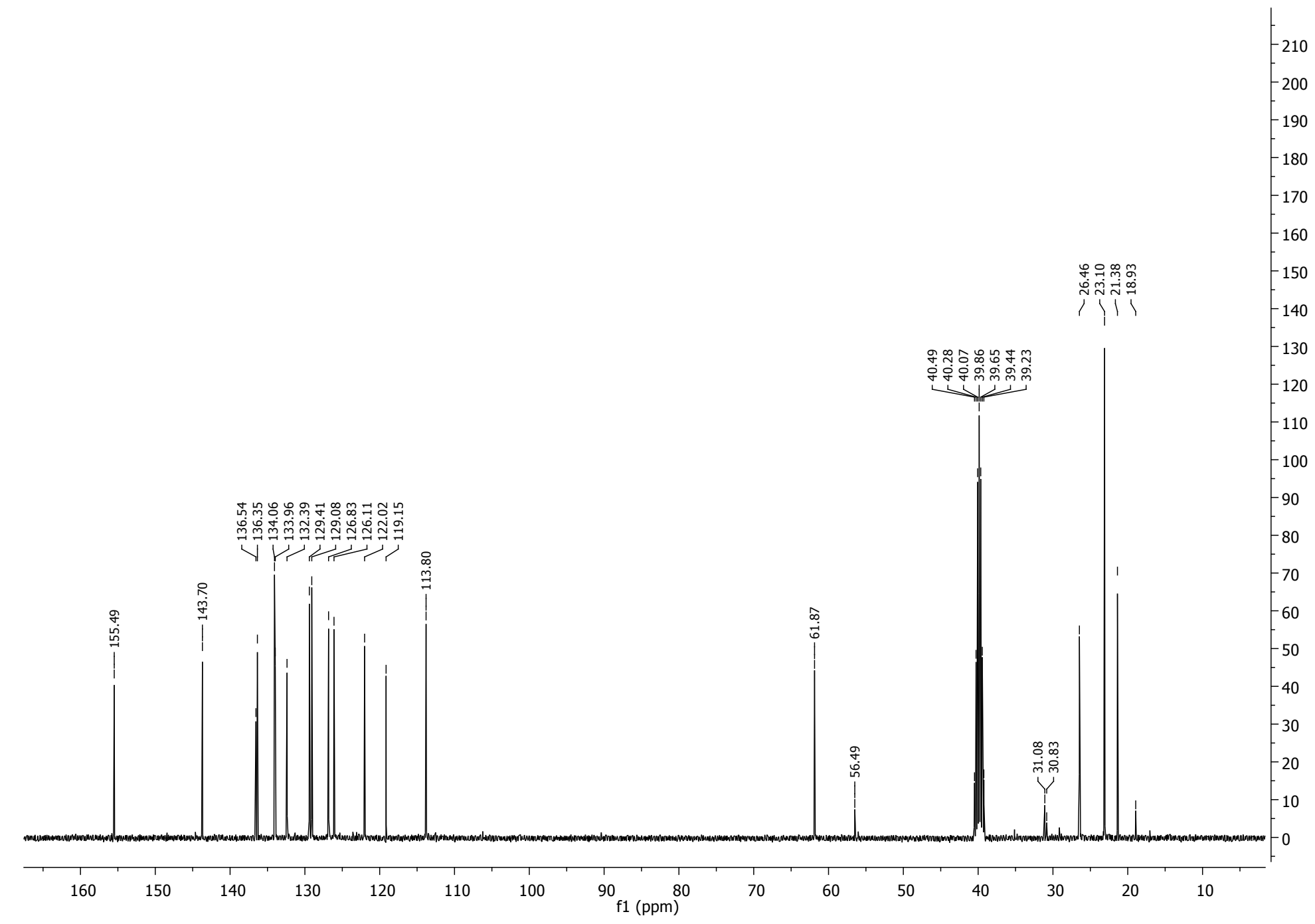

Figure S55. ${ }^{13} \mathrm{C}$ NMR spectrum (100 MHz, DMSO- $\left.d_{6}\right)$ of compound (1-(2-bromophenyl)-4-((2-isopropyl-5-methylphenoxy)methyl)-1 $H$-1,2,3-triazole) (3f). 


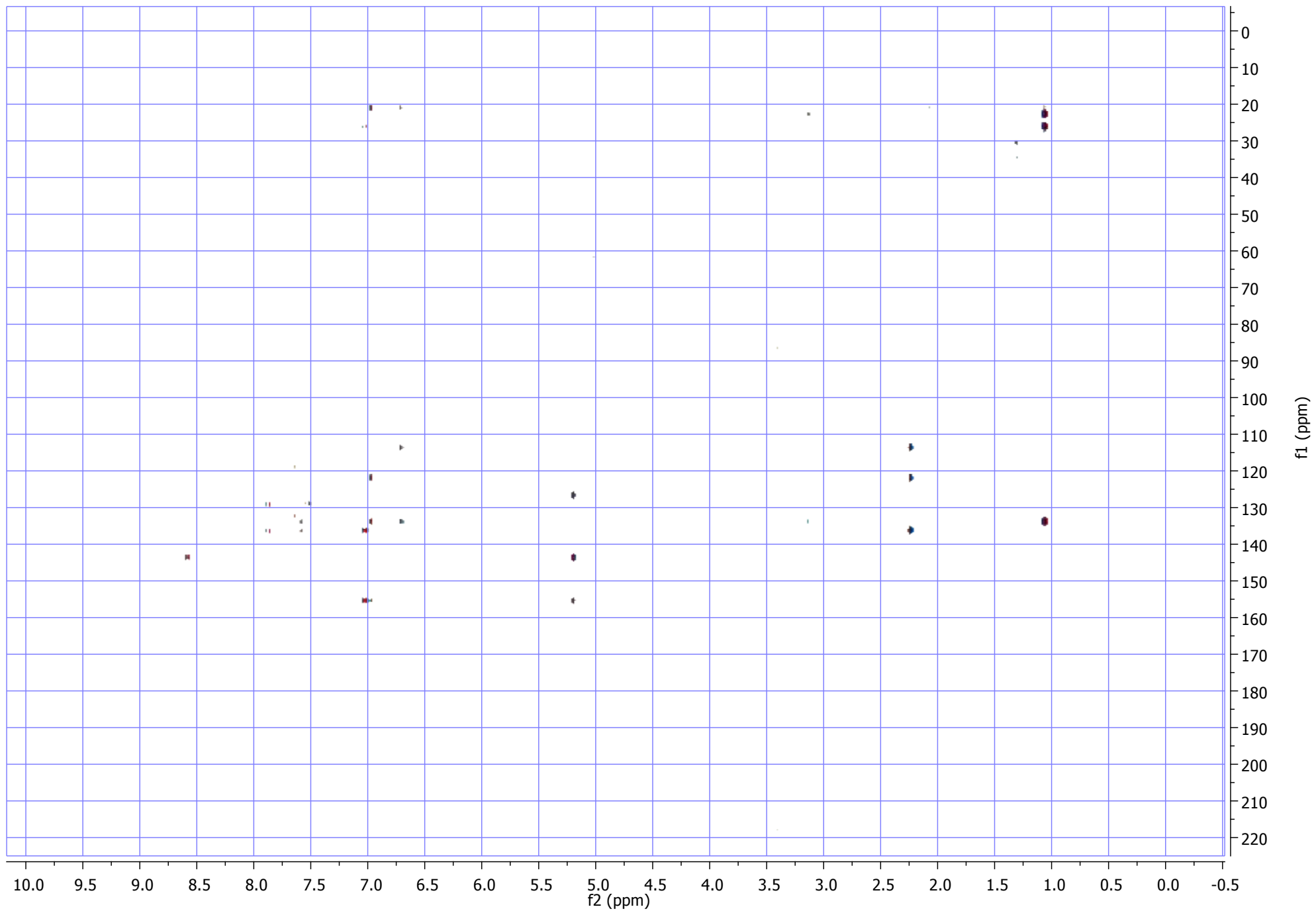

Figure S56 - HMBC spectrum (400 MHz, DMSO- $d_{6}$ ) of the compound (1-(2-bromophenyl)-4-((2-isopropyl-5-methylphenoxy)methyl)-1H-1,2,3-triazole) (3f). 


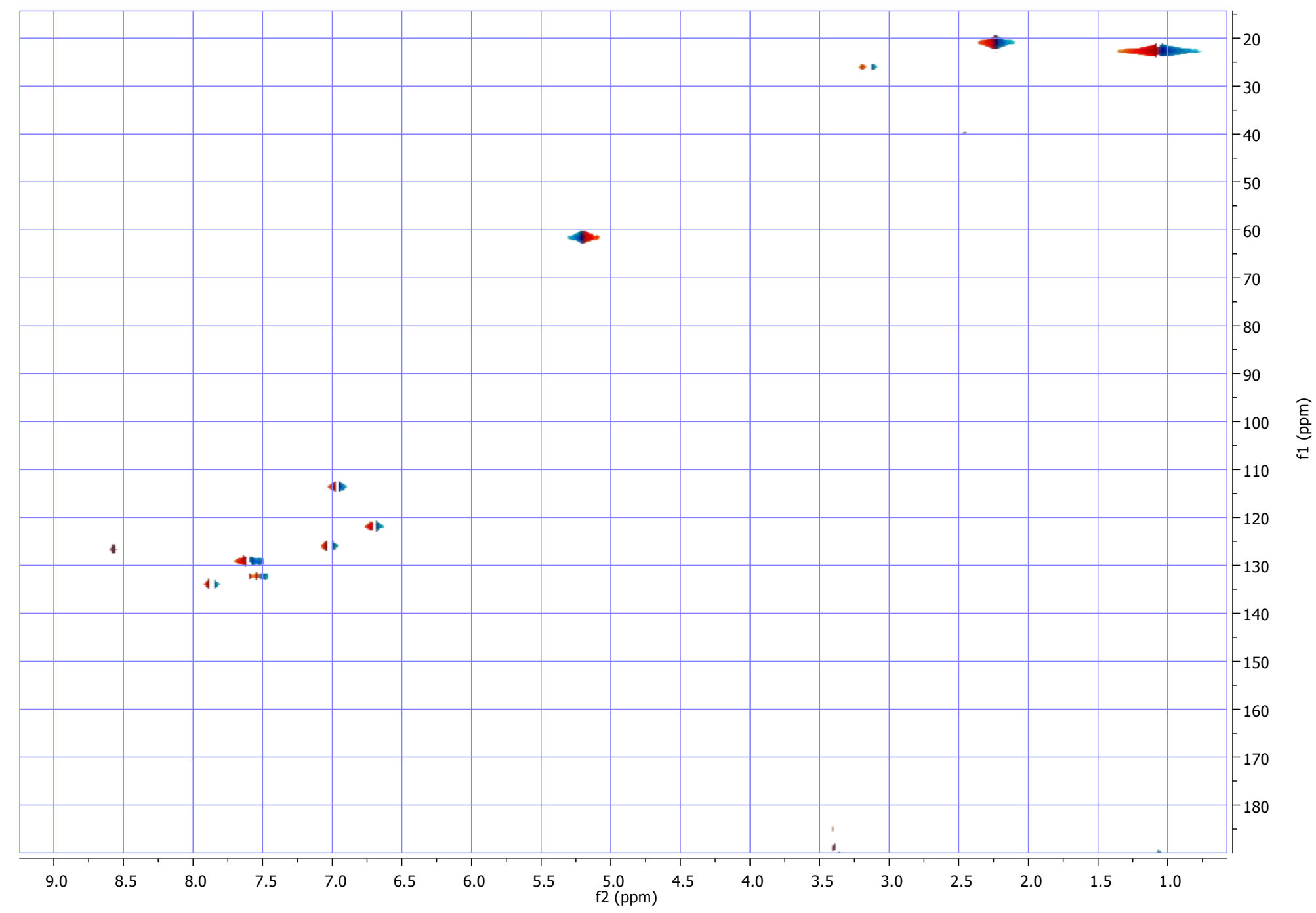

Figure S57 - HSQC spectrum (400 MHz, DMSO- $d_{6}$ ) of the compound (1-(2-bromophenyl)-4-((2-isopropyl-5-methylphenoxy)methyl)-1H-1,2,3-triazole) (3f). 


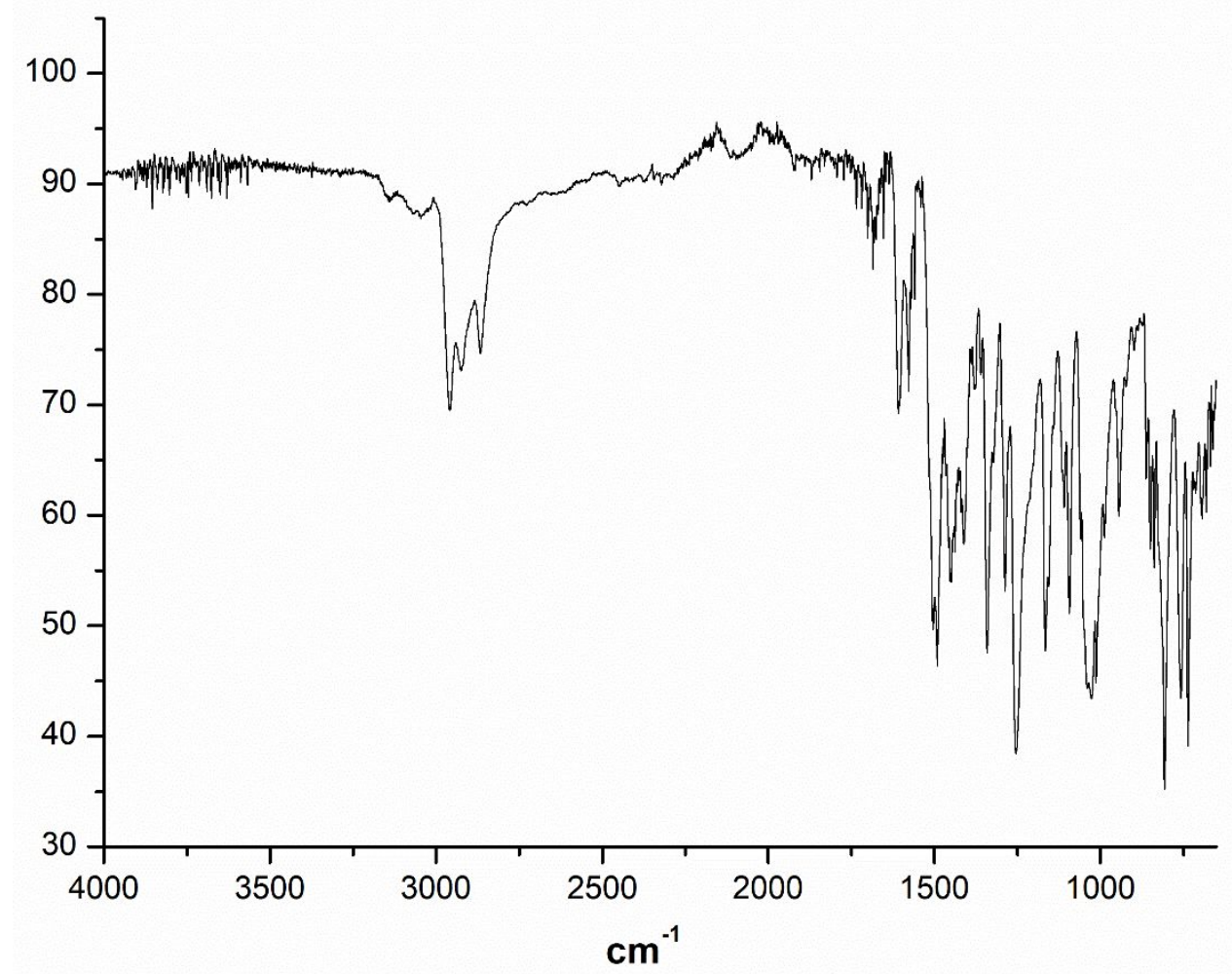

Figure S58. IR spectrum of compound $\mathbf{3 f}$. 


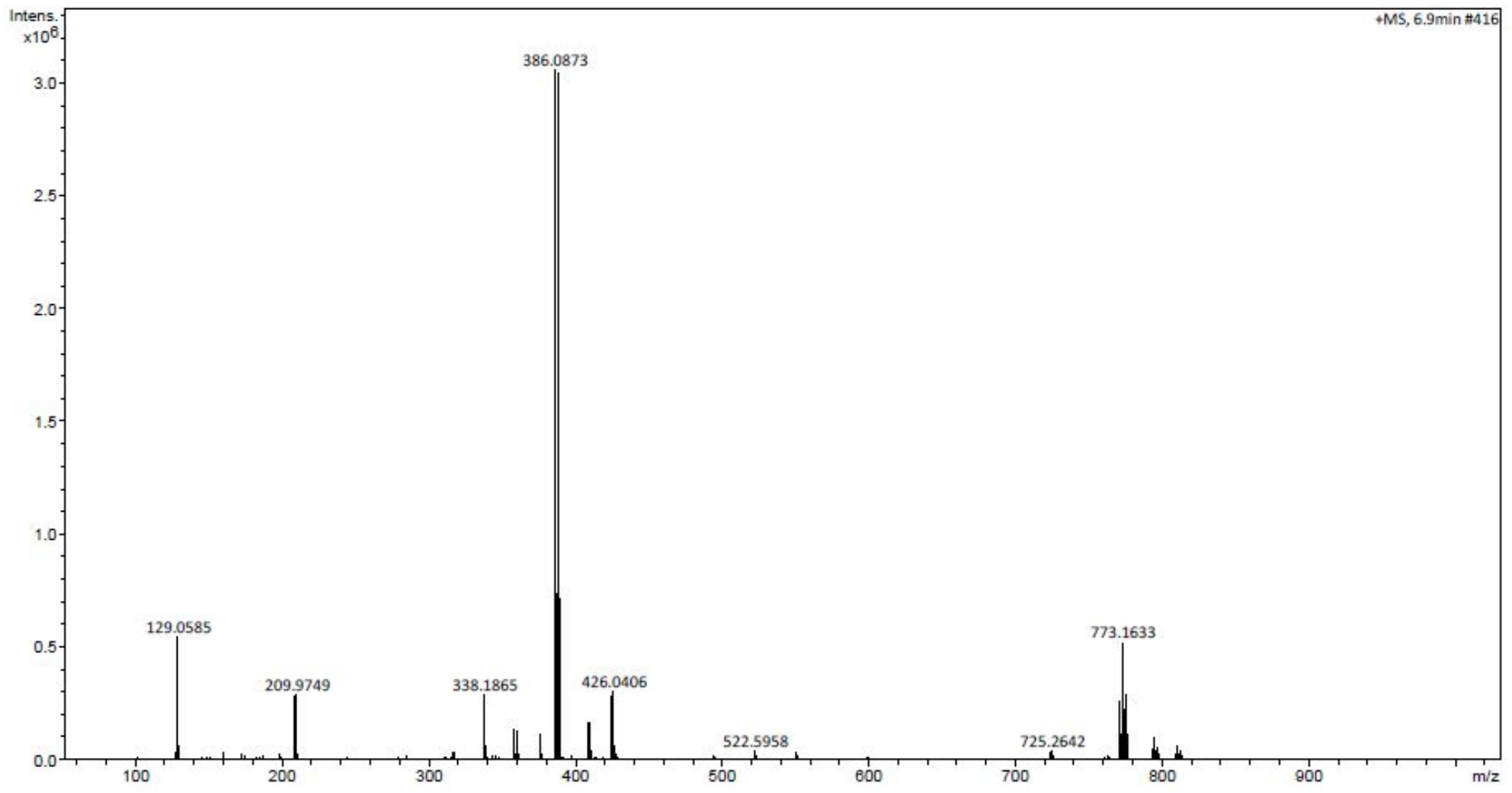

Figure S59. HRMS spectrum of compound $\mathbf{3 f}$ 


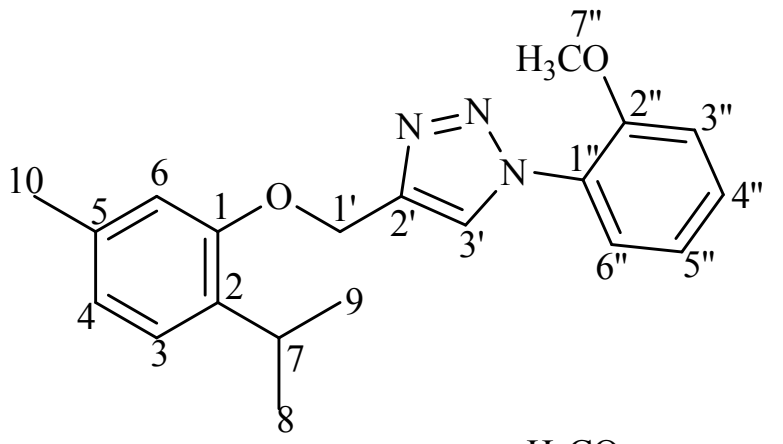

$3 g$

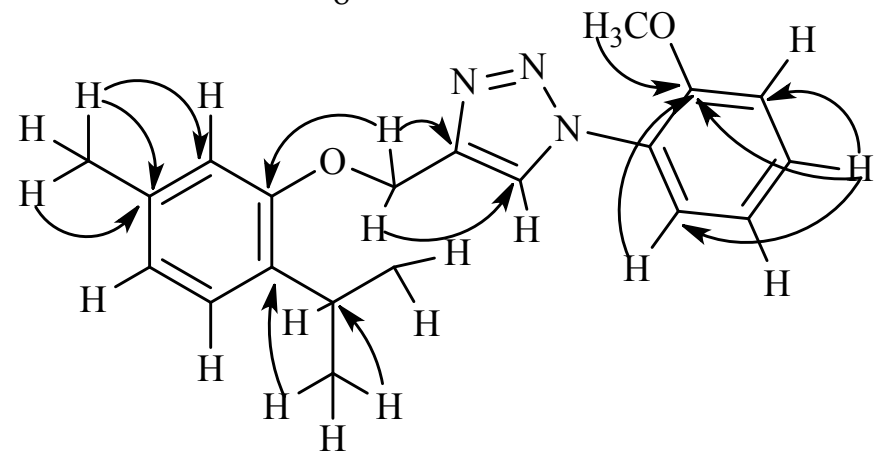

Figure S60. Compound 3g and main HMBC correlation $J$ in $\mathrm{Hz}$.
${ }^{1}$ H NMR (400 MHz, $1.08\left(6 \mathrm{H}, \mathrm{d}, \mathrm{CH}_{3}-8\right.$ and $\left.\mathrm{CH}_{3}-9\right) ; 2.26(3 \mathrm{H}, \mathrm{s}$, DMSO- $d_{6}$ )

$\left.\mathrm{CH}_{3}-10\right) ; 3.12-3.19(1 \mathrm{H}, \mathrm{m}, \mathrm{H}-7) ; 3.82(3 \mathrm{H}, \mathrm{s}, \mathrm{O}-$ $\left.\mathrm{CH}_{3}\right) ; 5.19\left(2 \mathrm{H}, \mathrm{s}, \mathrm{CH}_{2}-1^{\prime}\right) ; 6.71\left(1 \mathrm{H}, \mathrm{d}, J_{4,3} 7.82\right.$, $\mathrm{H}-4) ; 6.98(1 \mathrm{H}, \mathrm{s}, \mathrm{H}-6) ; 7.03\left(1 \mathrm{H}, \mathrm{d}, J_{3,4} 7.82, \mathrm{H}-\right.$ 3); $7.12\left(1 \mathrm{H}, \mathrm{t}, J_{5}, 6^{\prime}, 8.60, \mathrm{H}-5{ }^{\prime}\right) ; 7.29(1 \mathrm{H}, \mathrm{d}$,

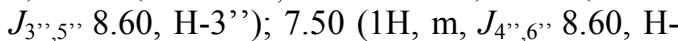
4"'); $7.62\left(1 \mathrm{H}, \mathrm{dl}, J_{6}\right.$, ,3, 8.60, H-6"'); $8.51(1 \mathrm{H}$, s, H-triazole).

${ }^{13}$ C NMR (100 MHz, 21.39 (C-10); 23.06 (C-8, C-9); 26.47 (C-7); DMSO- $d_{6}$ ) $56.52\left(\mathrm{O}-\mathrm{CH}_{3}\right) ; 61.82\left(\mathrm{C}-1\right.$ '); $113.46\left(\mathrm{C}-3{ }^{\prime}\right)$; 113.67 (C-6); 121.34 (C-5'); 121.91 (C-4); 126.05 (C-6'); 126.08 (C-3); 126.63 (C-3'); 131.13 (C-4"'); 133.87 (C-2); 136.31 (C-5); 143.42 (C-2'); 151.59 (C-2’'); 155.50 (C-1)

Infra-red

2960 (C-H in $\mathrm{CH}_{2}-1$ ', $\left.\mathrm{CH}_{3}-8, \mathrm{CH}_{3}-9, \mathrm{CH}_{3}-10\right)$; 2871 (C-H in $\mathrm{CH}-7) ; 1252$ and 1022 (ether in $\mathrm{CH}_{2}-1$ ' and $\mathrm{C}-1$ or / and $\mathrm{CH}_{3}-7$ ', and C-2',

Mass spectrometry HRESIMS $[\mathrm{M}+\mathrm{H}]^{+}$Found: 338.1861 Calc. for $\mathrm{C}_{20} \mathrm{H}_{24} \mathrm{~N}_{3} \mathrm{O}_{2}^{+}$: 338.1869 .

Table S10. Spectroscopic data for compound $\mathbf{3 g}$. 

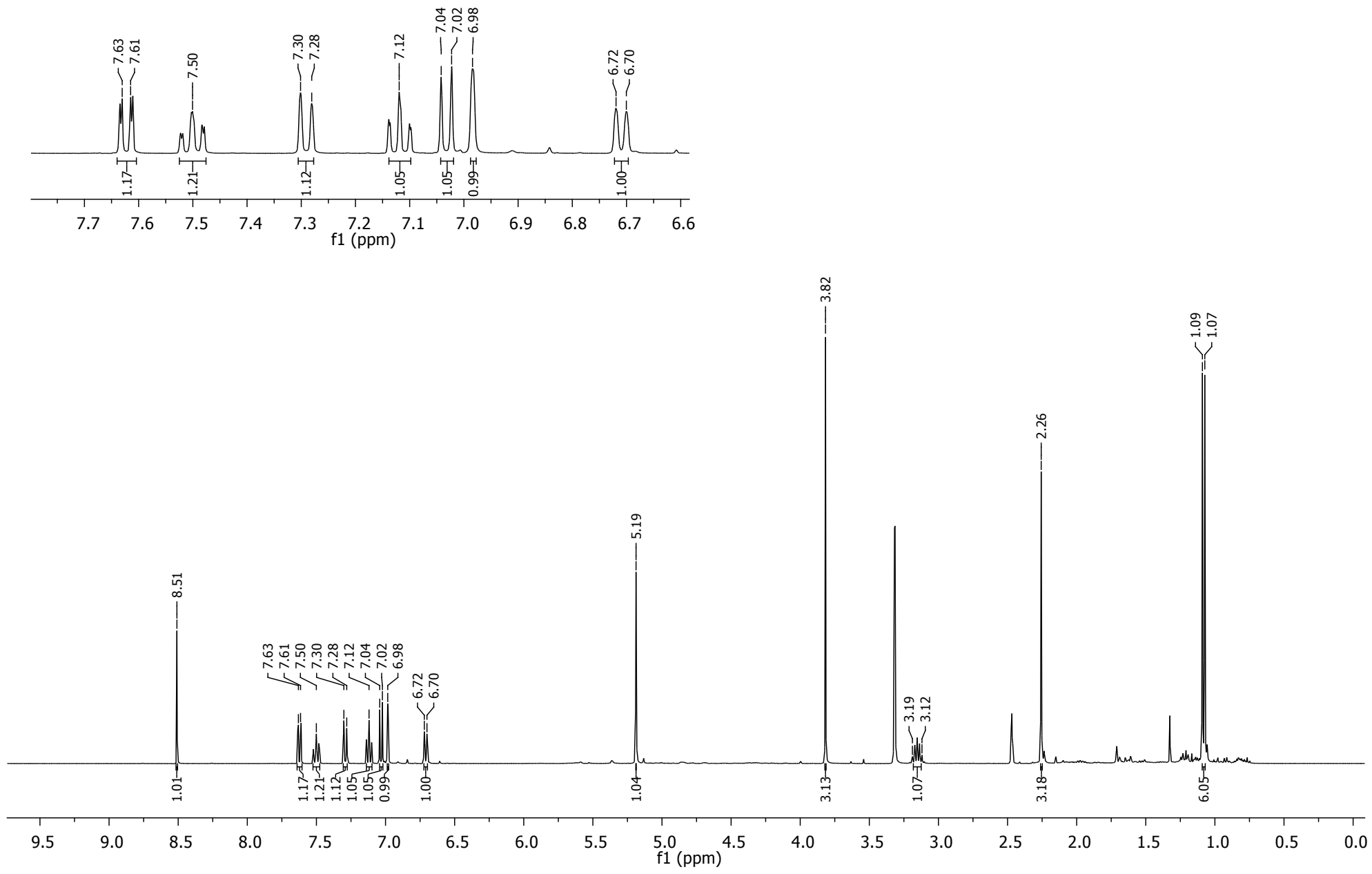

Figure S61. ${ }^{1} \mathrm{H}$ NMR spectrum (400 MHz, DMSO- $\left.d_{6}\right)$ of compound (4-((2-isopropyl-5-methylphenoxy)methyl)-1-(2-methoxyphenyl)-1H-1,2,3-triazole) (3g). 


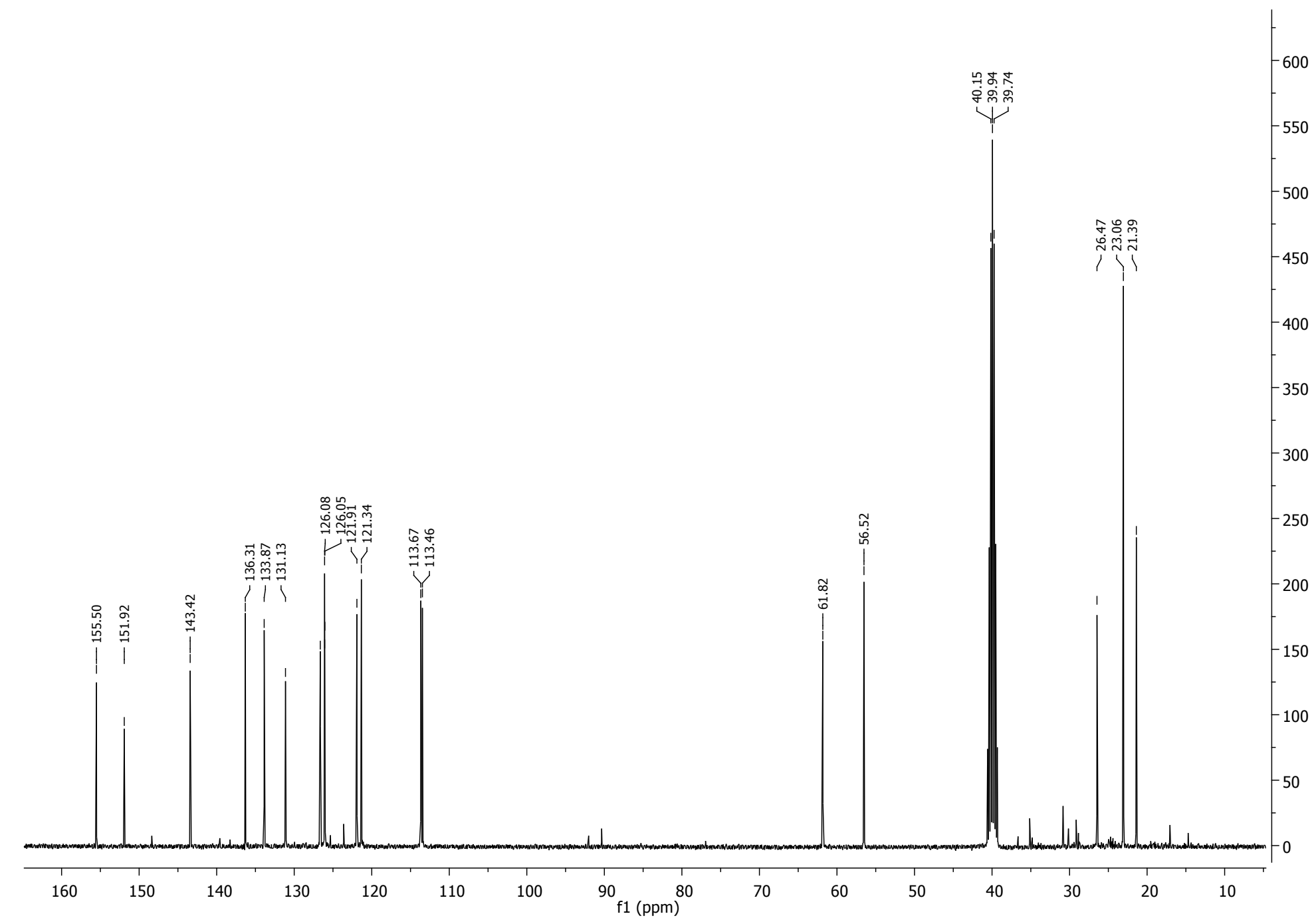

Figure S62. ${ }^{13} \mathrm{C}$ NMR spectrum (100 MHz, DMSO- $\left.d_{6}\right)$ of compound (4-((2-isopropyl-5-methylphenoxy)methyl)-1-(2-methoxyphenyl)-1H-1,2,3-triazole) (3g). 


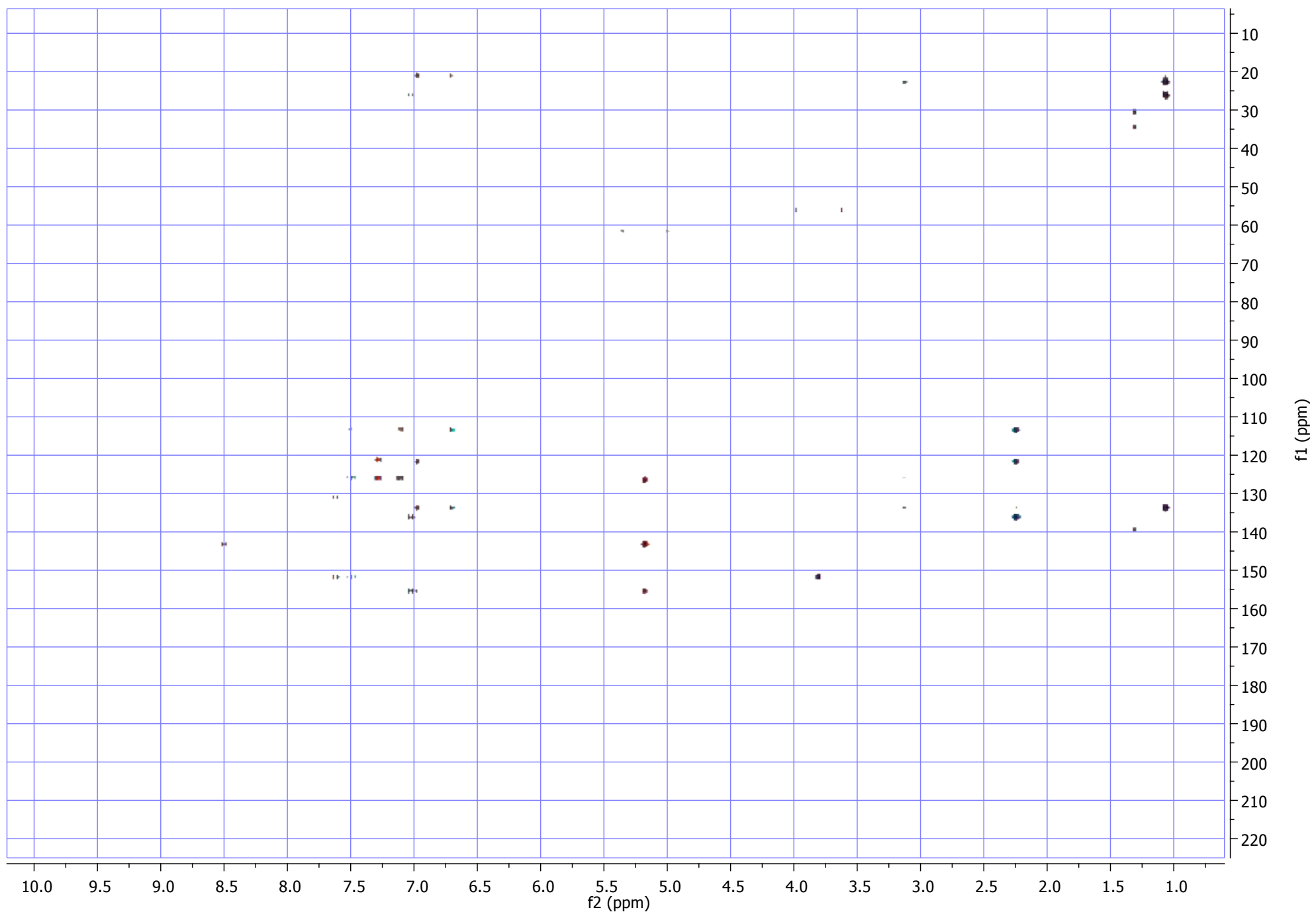

Figure S63 - HMBC spectrum (400 MHz, DMSO- $d_{6}$ ) of the compound (4-((2-isopropyl-5-methylphenoxy)methyl)-1-(2-methoxyphenyl)-1H-1,2,3-triazole) (3g). 


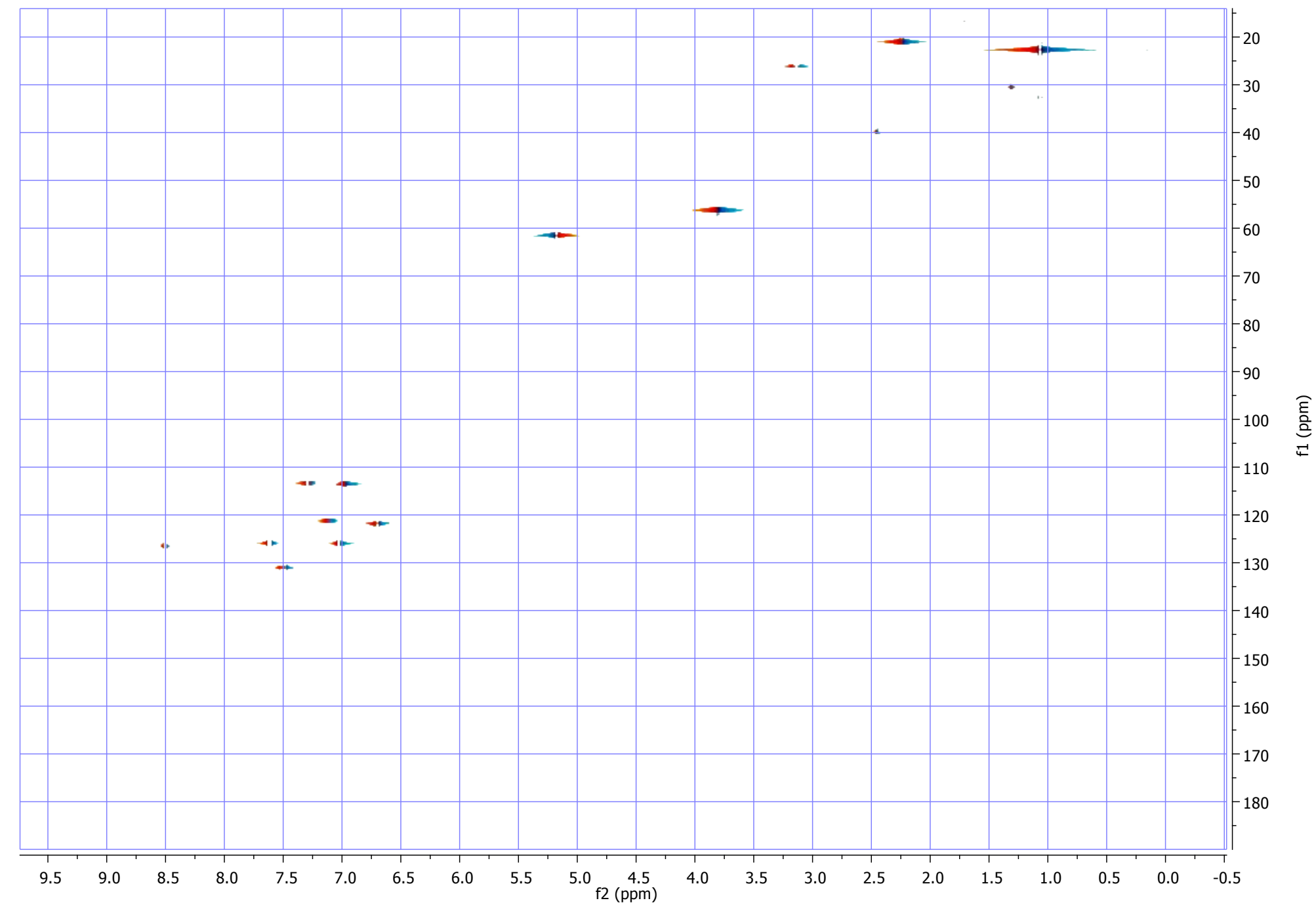

Figure S64 - HSQC spectrum (400 MHz, DMSO- $\left.d_{6}\right)$ of the compound (4-((2-isopropyl-5-methylphenoxy)methyl)-1-(2-methoxyphenyl)-1H-1,2,3-triazole) (3g). 


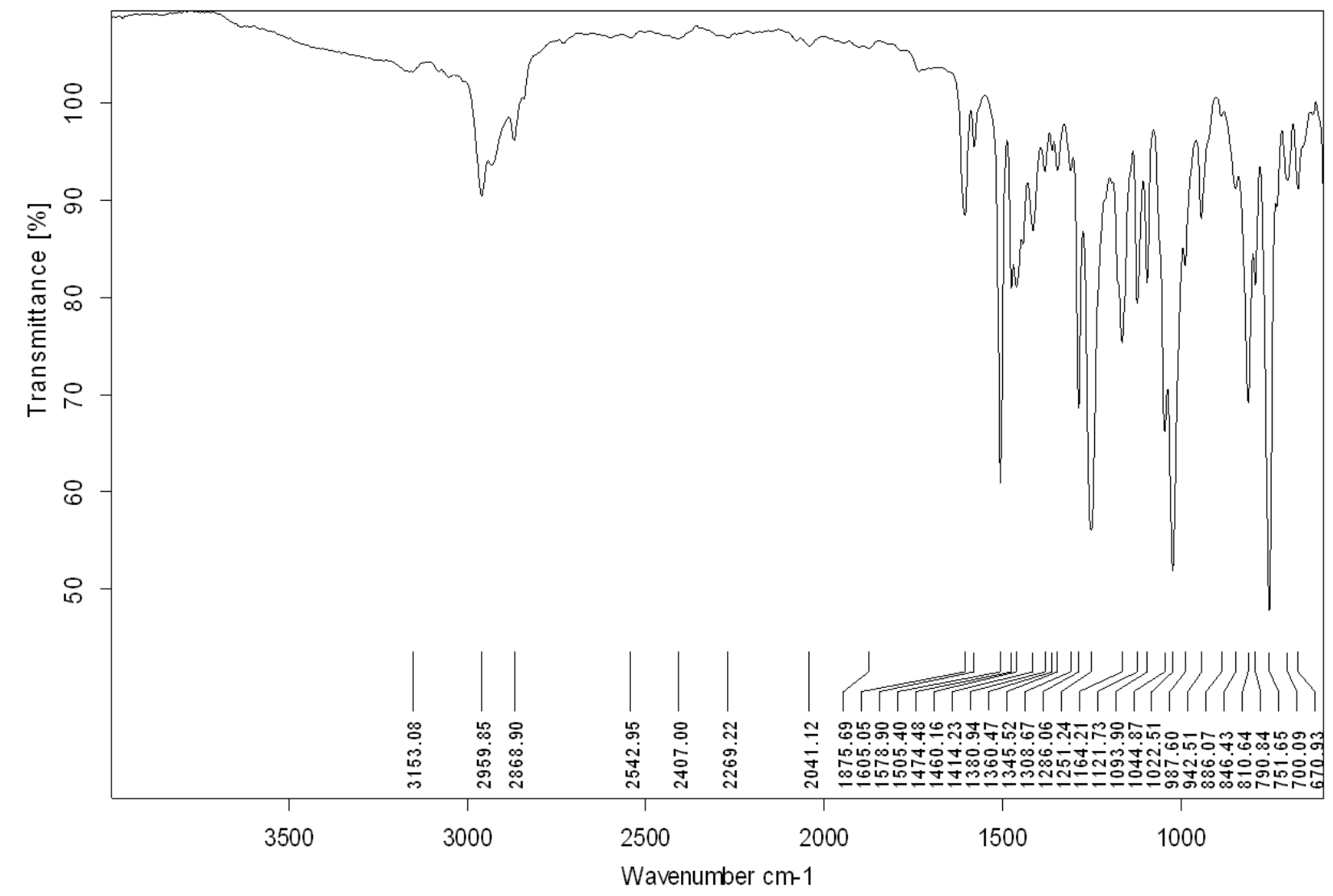

\begin{tabular}{|lll|l|}
\hline C:Program FilesIOPUS_65IMeasIPedrol16112020IRayma_42.0 $\quad$ Rayma_42 $\quad$ pasta_gel & $16 / 11 / 2020$ \\
\hline
\end{tabular}

Page 1/1

Figure S65. IR spectrum of compound $\mathbf{3 g}$. 


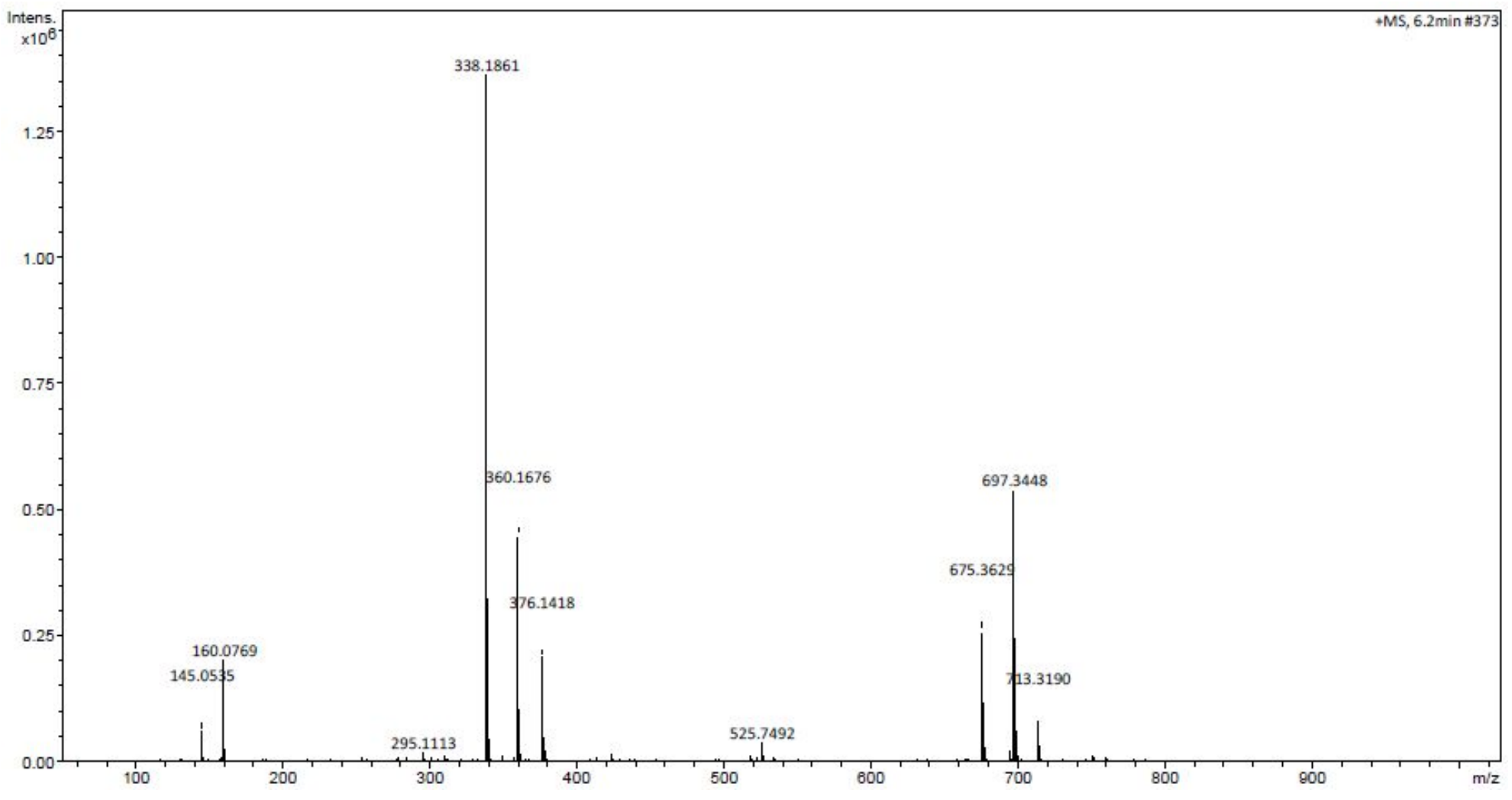

Figure S66. HRMS spectrum of compound 3g. 
<smiles>C=C(COc1cc(C)ccc1C(C)C)N=N</smiles>

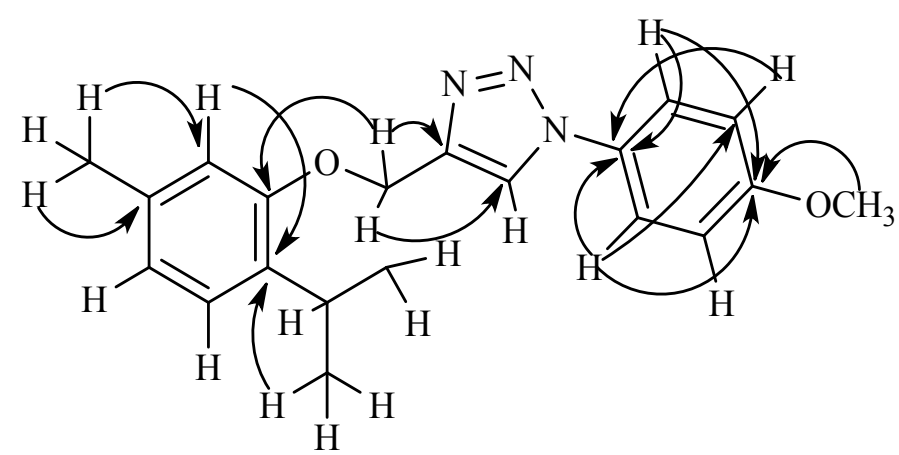

Figure S67. Compound $\mathbf{3 h}$ and main $\mathrm{HMBC}$ correlation $J$ in $\mathrm{Hz}$.
${ }^{1} \mathrm{H}$ NMR (400 MHz, $0.94\left(6 \mathrm{H}, \mathrm{d}, \mathrm{CH}_{3}-8\right.$ and $\left.\mathrm{CH}_{3}-9\right) ; 2.20(3 \mathrm{H}, \mathrm{s}$, DMSO- $d_{6}$ ) $\left.\mathrm{CH}_{3}-10\right) ; 2.78-2.86(1 \mathrm{H}, \mathrm{m}, \mathrm{H}-7) ; 3.79(3 \mathrm{H}, \mathrm{s}, \mathrm{O}-$ $\left.\mathrm{CH}_{3}\right) ; 5.17\left(2 \mathrm{H}, \mathrm{s}, \mathrm{CH}_{2}-1\right.$ ' $) ; 6.70\left(1 \mathrm{H}, \mathrm{d}, J_{4,3} 7.82\right.$ $\mathrm{H}-4) ; 6.77$ (1H, s, H-6); $6.99\left(1 \mathrm{H}, \mathrm{d}, J_{3,4} 7.82, \mathrm{H}-\right.$ 3); $7.09\left(2 \mathrm{H}, \mathrm{dd}, J_{2},{ }_{3}, 7.43, \mathrm{H}-2\right.$ ', and H-6"'); 7.53 (2H, dd, $J_{5},, 6^{\prime}, 7.43, \mathrm{H}-3$ ', and H-5', $) ; 7.98$ (1H, s, H-triazole).

${ }^{13}$ C NMR (100 MHz, 21.28 (C-10); 23.11 (C-8, C-9); 25.94 (C-7); DMSO-d D $^{2}$ $56.00\left(\mathrm{O}-\mathrm{CH}_{3}\right) ; 58.81(\mathrm{C}-1$ '); $113.34(\mathrm{C}-6)$; 115.11 (C-2" and C-6" '); 122.34 (C-4); 126.15 (C-3); 126.41 (C-3'' and C-5' '); 129.34 (C-1''); 133.84 (C-2'); 134.29 (C-2); 134.85 (C-3'); 136.34 (C-5); 154.68 (C-1); 160.39 (C-4'”).

Infra-red $\left(\mathrm{cm}^{-1}\right)$

2960 (C-H in $\mathrm{CH}_{2}-1$ ', $\mathrm{CH}_{3}-8, \mathrm{CH}_{3}-9, \mathrm{CH}_{3}-10$ ); 2871 (C-H in $\mathrm{CH}-7$ ); 1252 and 1022 (ether in $\mathrm{CH}_{2}-1$ ' and $\mathrm{C}-1$ or / and $\mathrm{CH}_{3}-7$ ', and C-4'')

Mass spectrometry HRESIMS $[\mathrm{M}+\mathrm{H}]^{+}$Found: 338.1863 Calc. for $\mathrm{C}_{20} \mathrm{H}_{24} \mathrm{~N}_{3} \mathrm{O}_{2}^{+}$: 338.1869.

Table S11. Spectroscopic data for compound $\mathbf{3 h}$. 

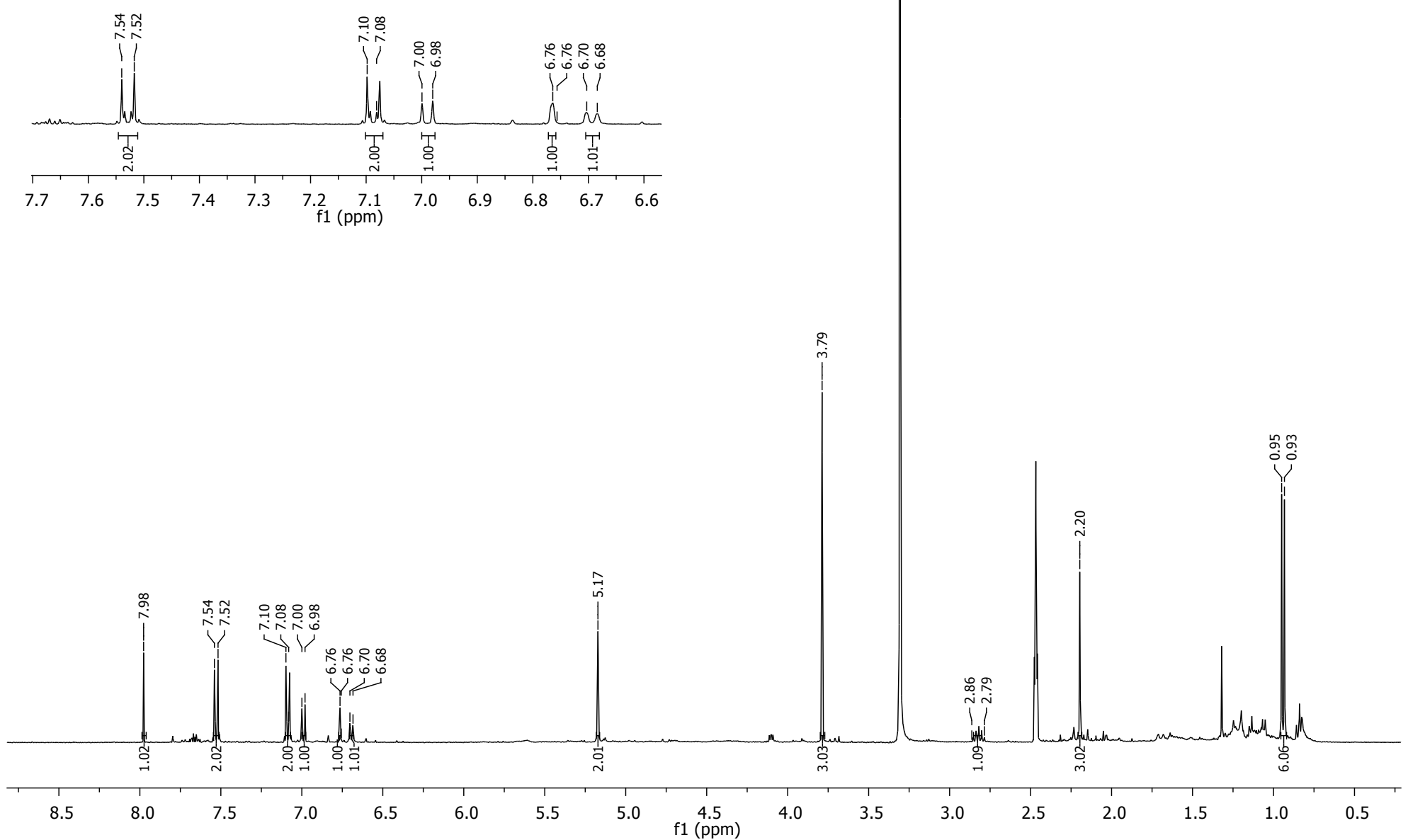

Figure S68. ${ }^{1} \mathrm{H}$ NMR spectrum (400 MHz, DMSO- $\left.d_{6}\right)$ of compound (4-((2-isopropyl-5-methylphenoxy)methyl)-1-(4-methoxyphenyl)-1 $H$-1,2,3-triazole) (3h). 


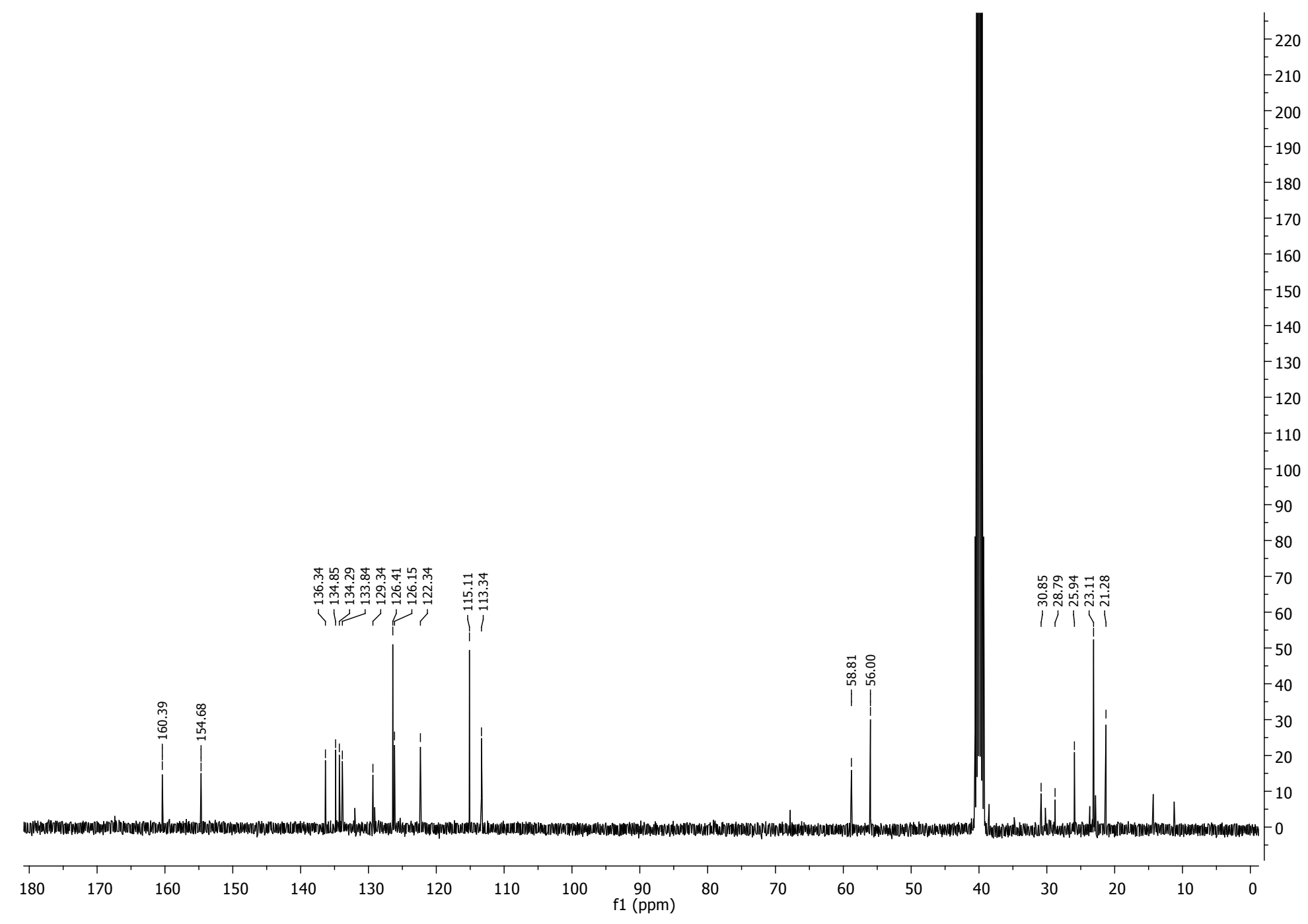

Figure S69. ${ }^{13} \mathrm{C}$ NMR spectrum (100 MHz, DMSO- $d_{6}$ ) of compound (4-((2-isopropyl-5-methylphenoxy)methyl)-1-(4-methoxyphenyl)-1 $H$-1,2,3-triazole) (3h). 


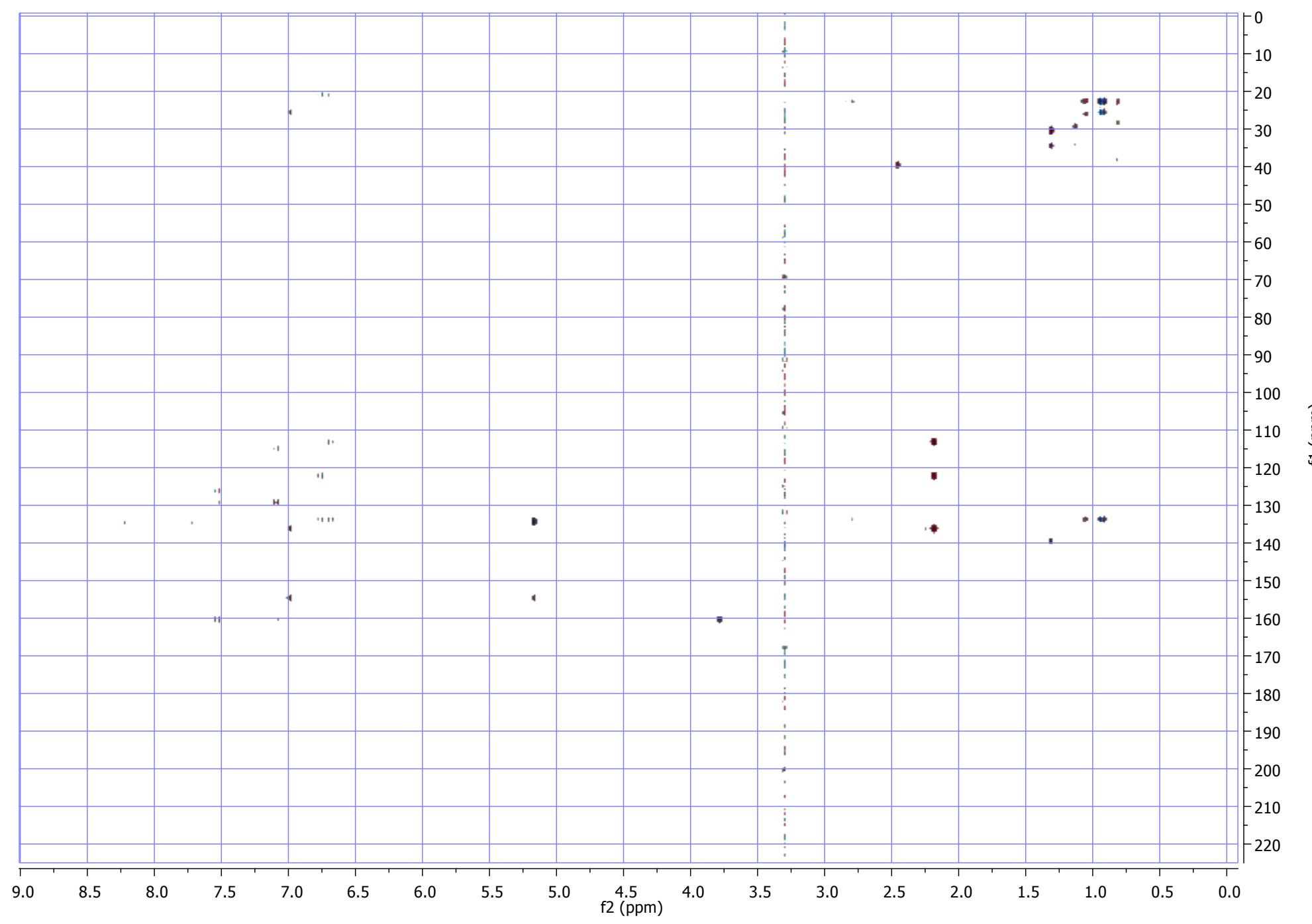

Figure S70 - HMBC spectrum (400 MHz, DMSO- $d_{6}$ ) of the compound (4-((2-isopropyl-5-methylphenoxy)methyl)-1-(4-methoxyphenyl)-1H-1,2,3-triazole) (3h). 


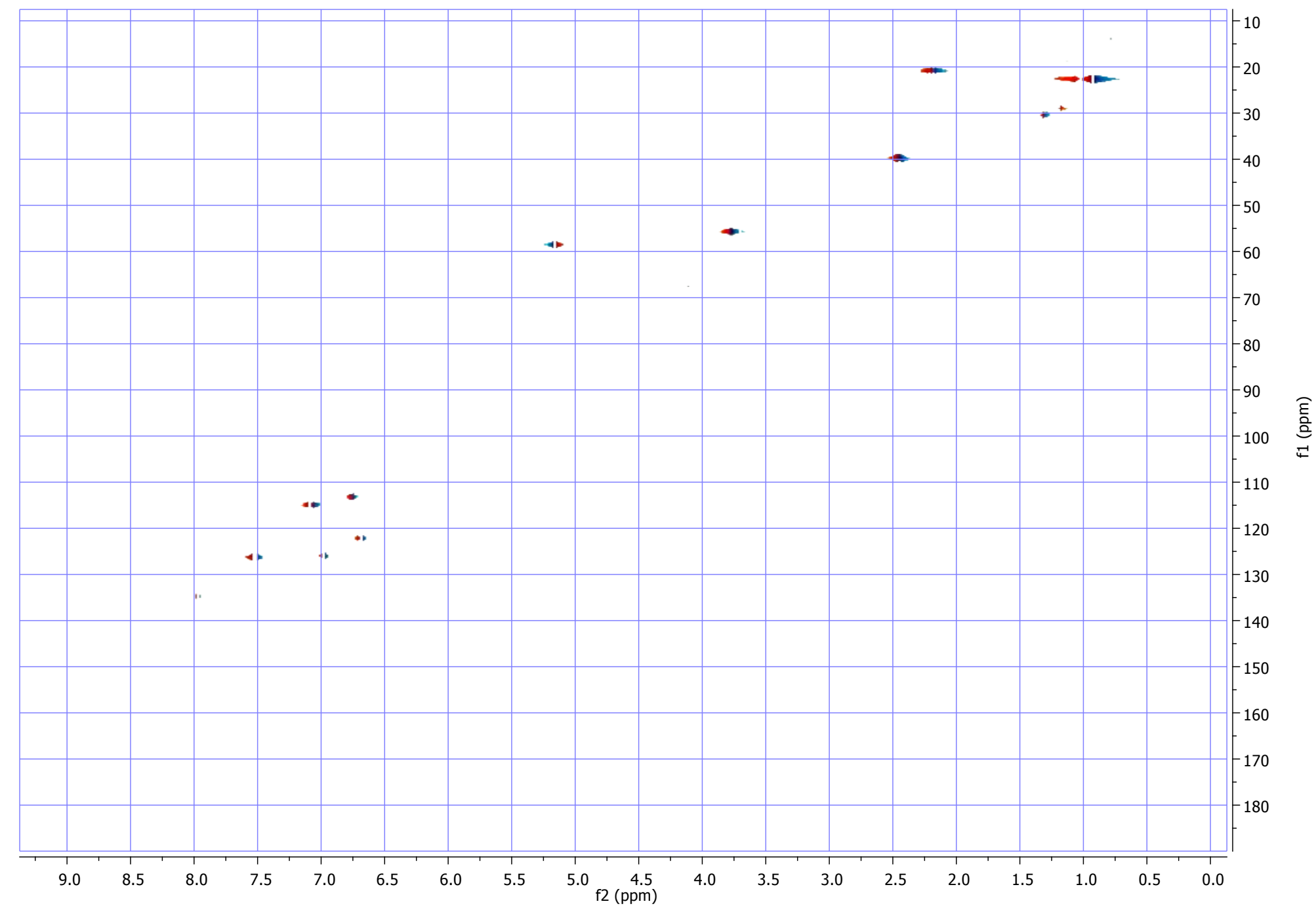

Figure S71 - HSQC spectrum (400 MHz, DMSO- $d_{6}$ ) of the compound (4-((2-isopropyl-5-methylphenoxy)methyl)-1-(4-methoxyphenyl)-1H-1,2,3-triazole) (3h). 


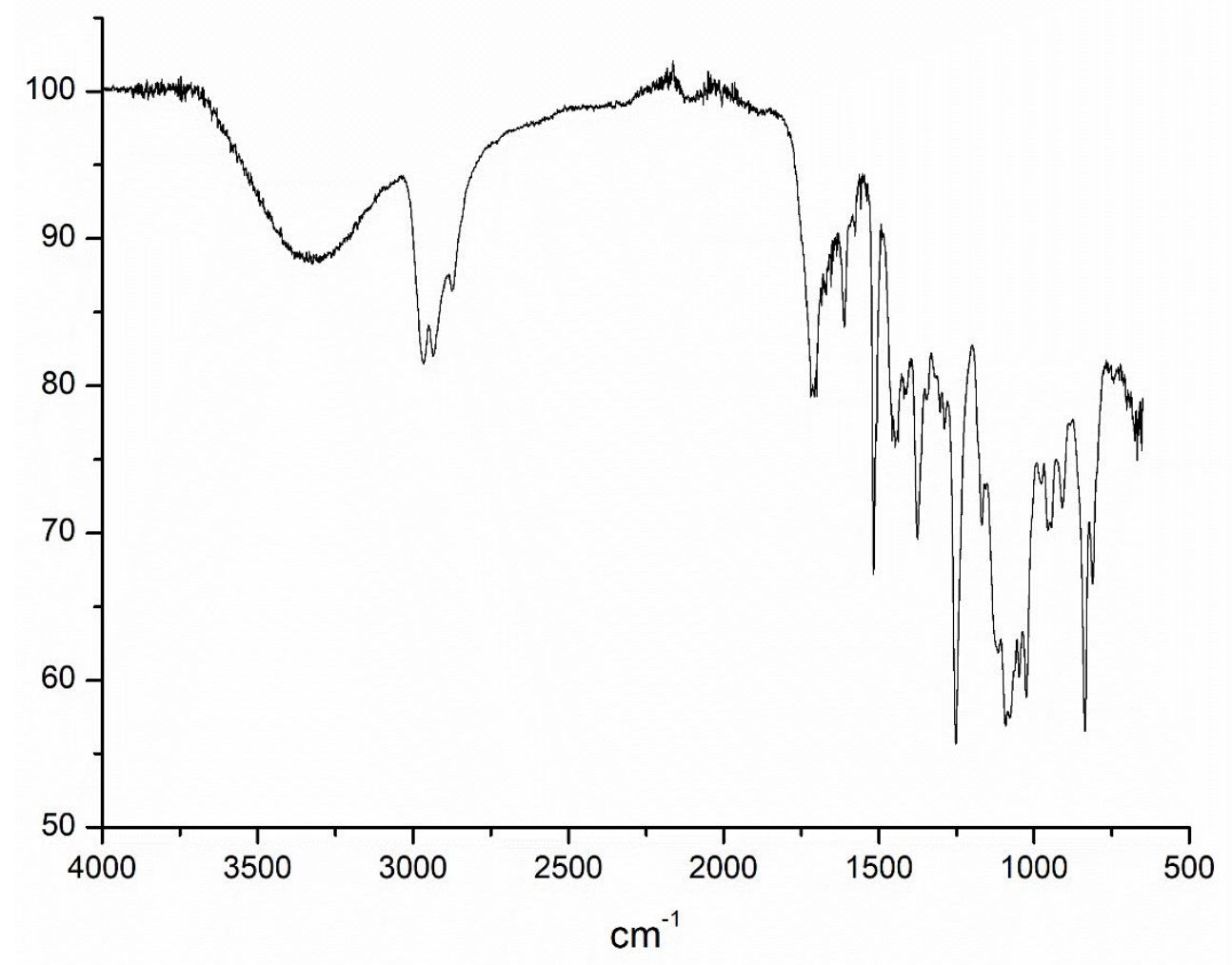

Figure S72. IR spectrum of compound $\mathbf{3 h}$. 


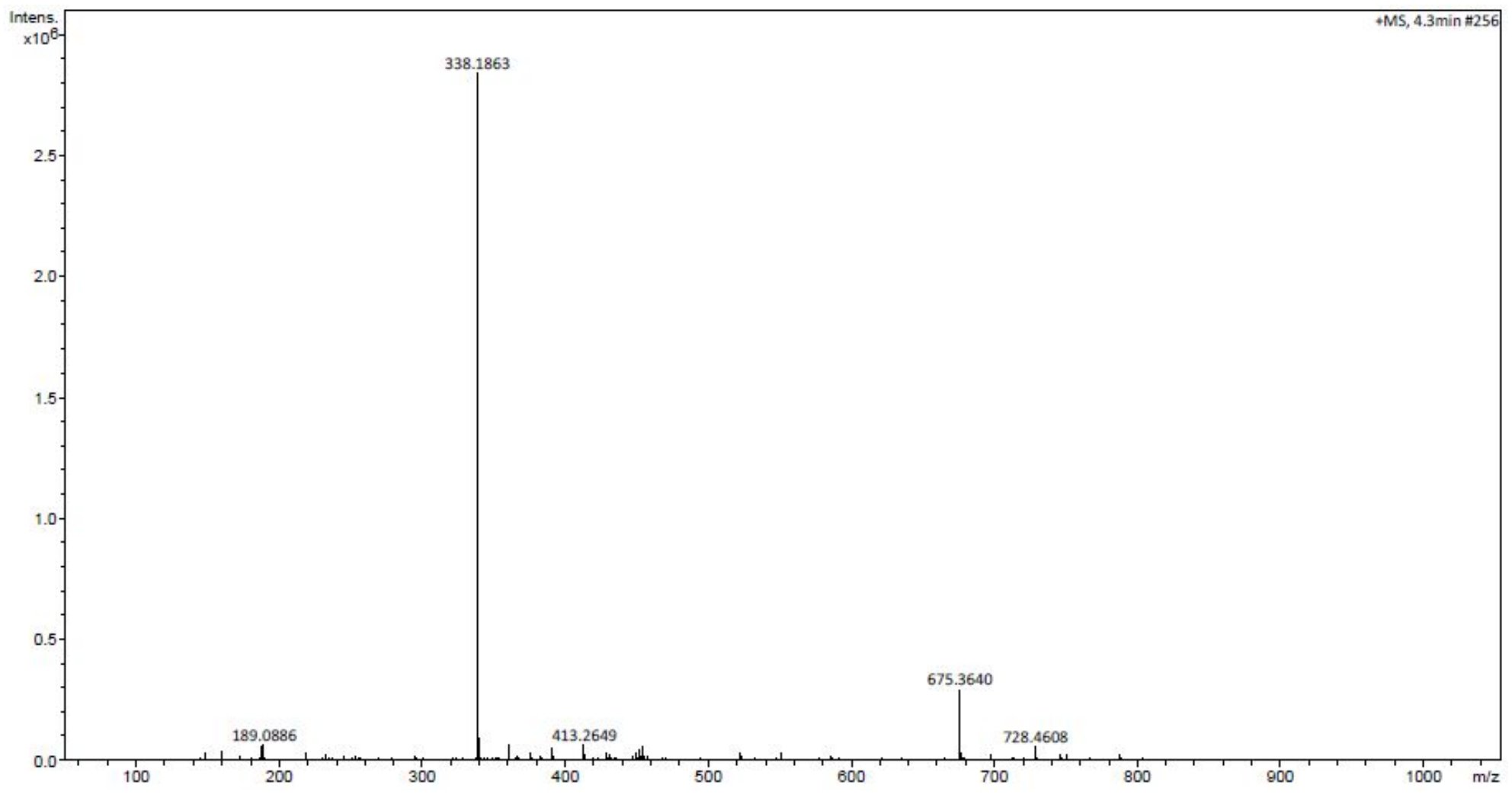

Figure S73. HRMS spectrum of compound $\mathbf{3 h}$. 
<smiles>[2H]c1ccc(C(C)C)c(OCc2cn(-c3cccc(OC)c3)nn2)c1</smiles>

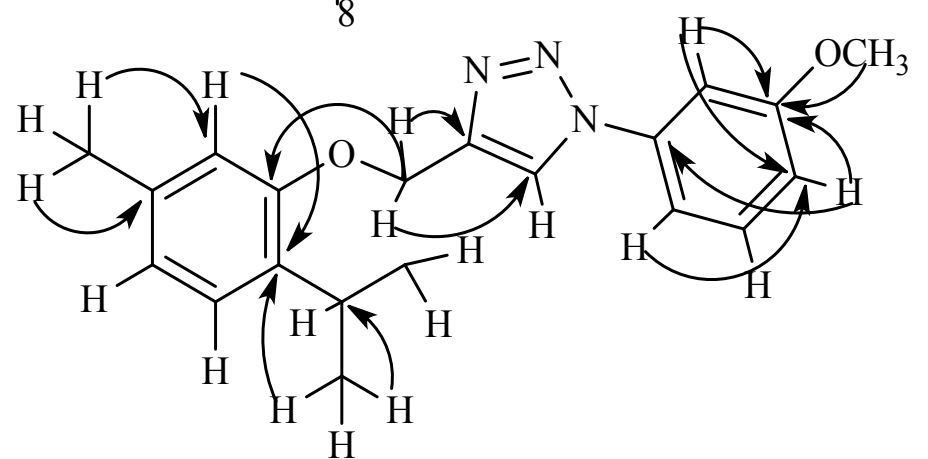

Figure S74. Compound 3i and main HMBC correlation $J$ in $\mathrm{Hz}$.
${ }^{1}$ H NMR (400 MHz, $1.06\left(6 \mathrm{H}, \mathrm{d}, \mathrm{CH}_{3}-8\right.$ and $\left.\mathrm{CH}_{3}-9\right) ; 2.24(3 \mathrm{H}, \mathrm{s}$, DMSO- $d_{6}$ )

 $\left.\mathrm{CH}_{3}\right) ; 5.16\left(2 \mathrm{H}, \mathrm{s}, \mathrm{CH}_{2}-1\right)$ ') $6.71\left(1 \mathrm{H}, \mathrm{d}, J_{4,3} 7.82\right.$ H-4); 6.96 (1H, s, H-6); 7.01-7.06 (2H, m, $J_{6},{ }^{\prime}$, $7.43, J_{34} 7.82, \mathrm{H}-6$ " ' and $\left.\mathrm{H}-3\right) ; 7.43\left(1 \mathrm{H}, \mathrm{d}, J_{2},{ }_{4}\right.$, 7.43, H-2"'); 7.45 (1H, t, $J_{5}$ "'6, 7.43, H-5"); 7.47 $\left(1 \mathrm{H}, \mathrm{d}, J_{4},{ }^{\prime}, 7.43, \mathrm{H}-4{ }^{\prime \prime}\right) ; 8.87(1 \mathrm{H}, \mathrm{s}, \mathrm{H}-$ triazole).

${ }^{13}$ C NMR (100 MHz， 21.35 (C-10); 23.08 (C-8, C-9); 26.35 (C-7); DMSO-d 6 ) $56.03\left(\mathrm{O}-\mathrm{CH}_{3}\right) ; 61.72\left(\mathrm{C}-1{ }^{\prime}\right) ; 106.20\left(\mathrm{C}-2^{\prime \prime}\right)$ 112.55 (C-4'); 113.61 (C-6); 114.88 (C-6”); 122.04 (C-4); 123.12 (C-3'); 126.12 (C-3); 131.37 (C-5"); $133.92(\mathrm{C}-2) ; 136.40(\mathrm{C}-5)$ 137.95 (C-1'); 144.63 (C-2'); 155.41 (C-1); 160.60 (C-3").

Infra-red $\left(\mathrm{cm}^{-1}\right)$

2955 (C-H in $\mathrm{CH}_{2}-1$ ', CH-7, $\mathrm{CH}_{3}-8, \mathrm{CH}_{3}-9$, $\mathrm{CH}_{3}-10$ ); 1232 and 1048 (ether in $\mathrm{CH}_{2}-1$ ' and $\mathrm{C}$ 1 or / and $\mathrm{CH}_{3}-7$ "' and $\mathrm{C}-3$ "').

Mass spectrometry HRESIMS $[\mathrm{M}+\mathrm{H}]^{+}$Found: 338.1861 Calc. for $\mathrm{C}_{20} \mathrm{H}_{24} \mathrm{~N}_{3} \mathrm{O}_{2}^{+}: 338.1869$.

Table S12. Spectroscopic data for compound 3i. 

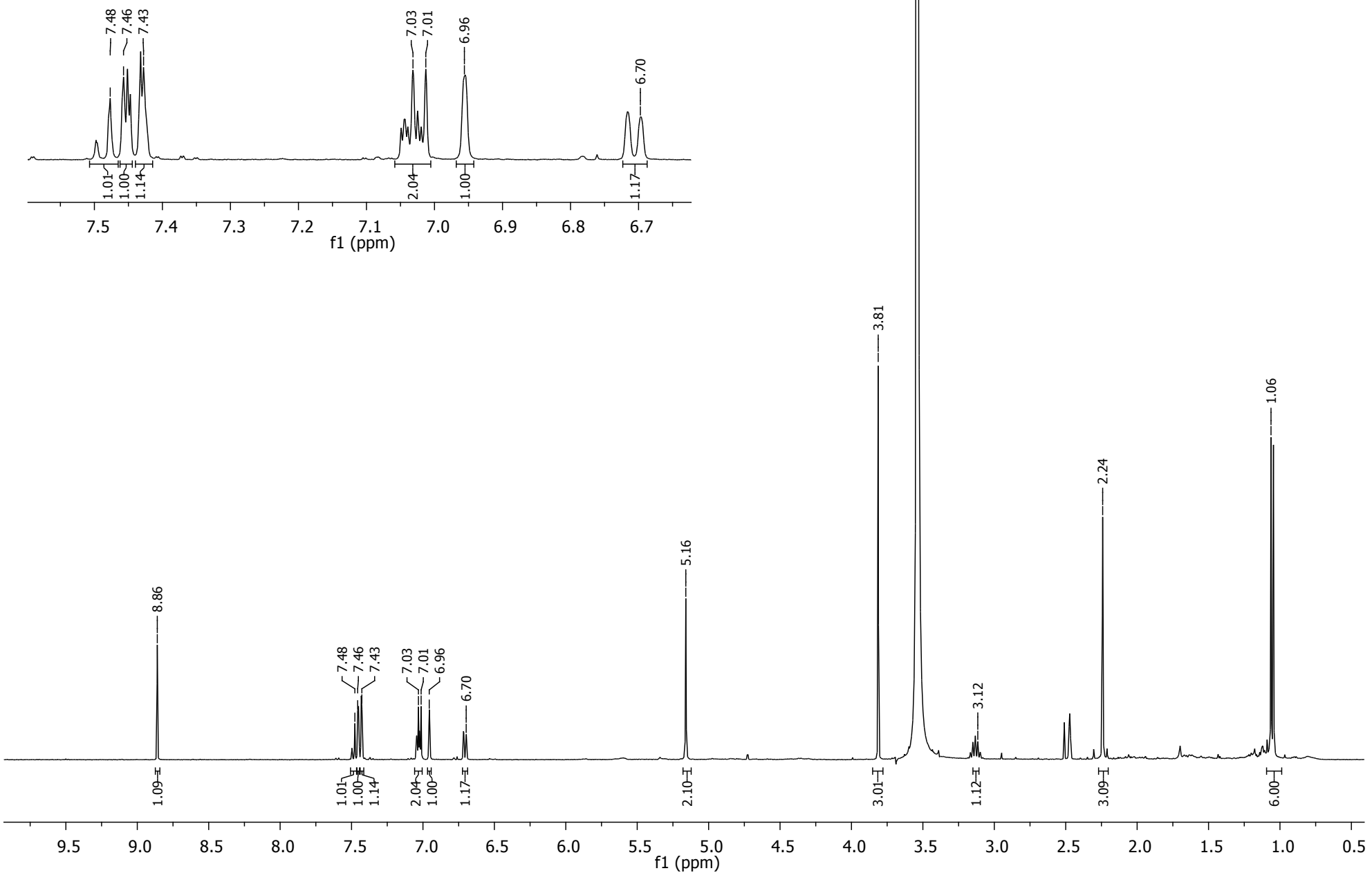

Figure S75. ${ }^{1} \mathrm{H}$ NMR spectrum (400 MHz, DMSO- $d_{6}$ ) of compound (4-((2-isopropyl-5-methylphenoxy)methyl)-1-(3-methoxyphenyl)-1H-1,2,3-triazole) (3i). 


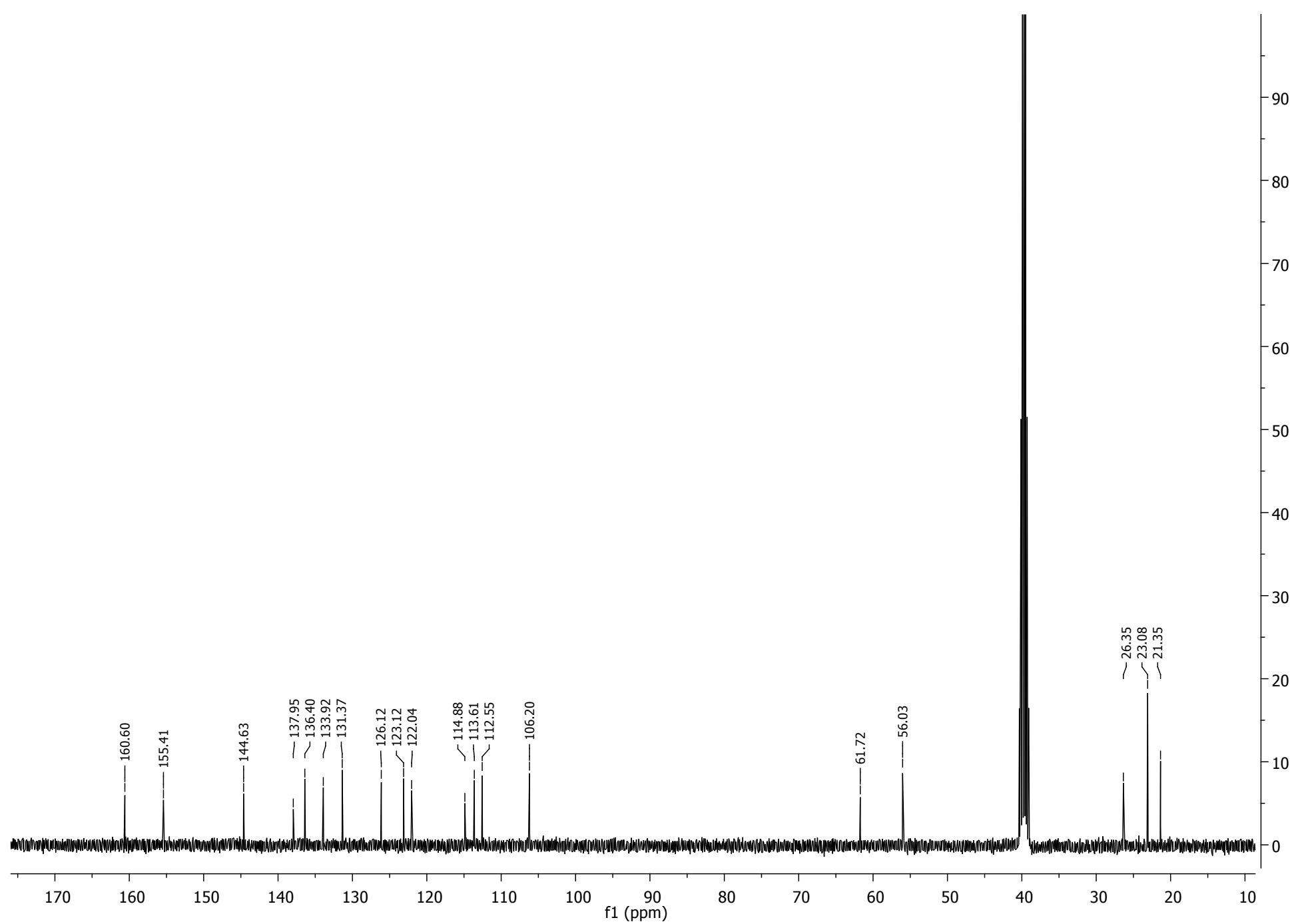

Figure S76. ${ }^{13} \mathrm{C}$ NMR spectrum (100 MHz, DMSO- $\left.d_{6}\right)$ of compound (4-((2-isopropyl-5-methylphenoxy)methyl)-1-(3-methoxyphenyl)-1H-1,2,3-triazole) (3i). 


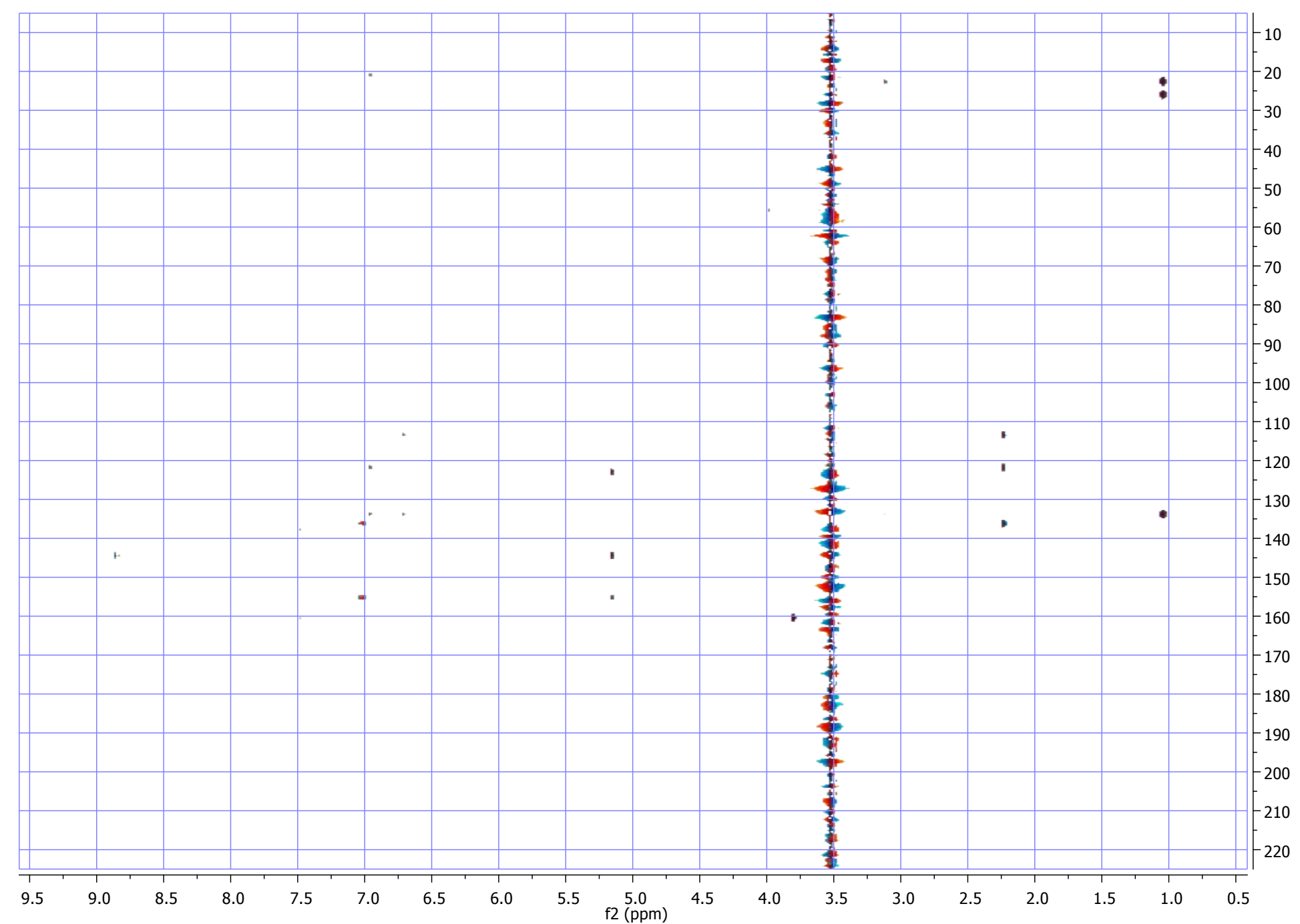

Figure S77 - HMBC spectrum (400 MHz, DMSO- $d_{6}$ ) of the compound (4-((2-isopropyl-5-methylphenoxy)methyl)-1-(3-methoxyphenyl)-1 $H$-1,2,3-triazole) (3i). 


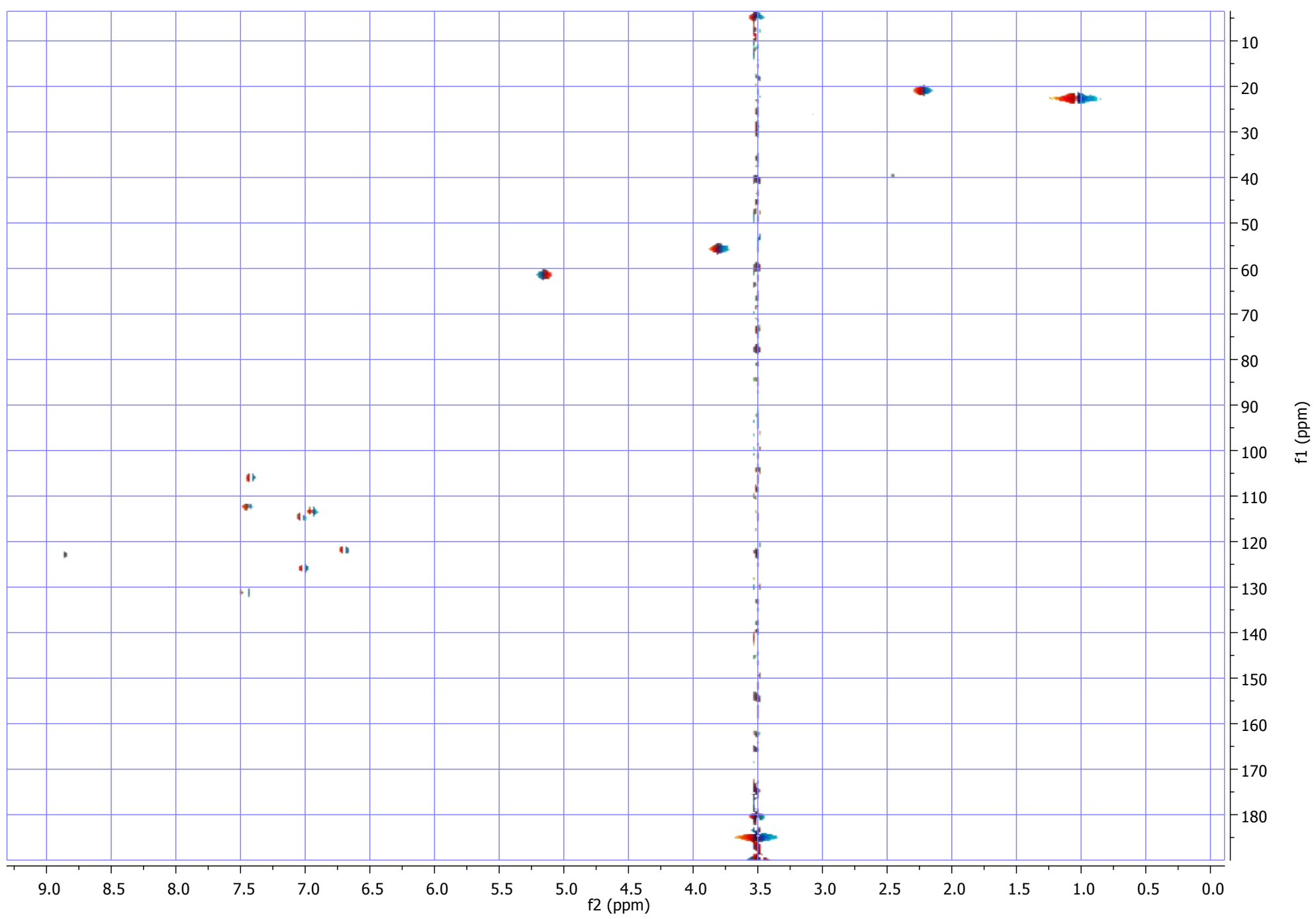

Figure S78 - HSQC spectrum (400 MHz, DMSO- $d_{6}$ ) of the compound (4-((2-isopropyl-5-methylphenoxy)methyl)-1-(3-methoxyphenyl)-1 $i-1,2,3$-triazole) (3i). 


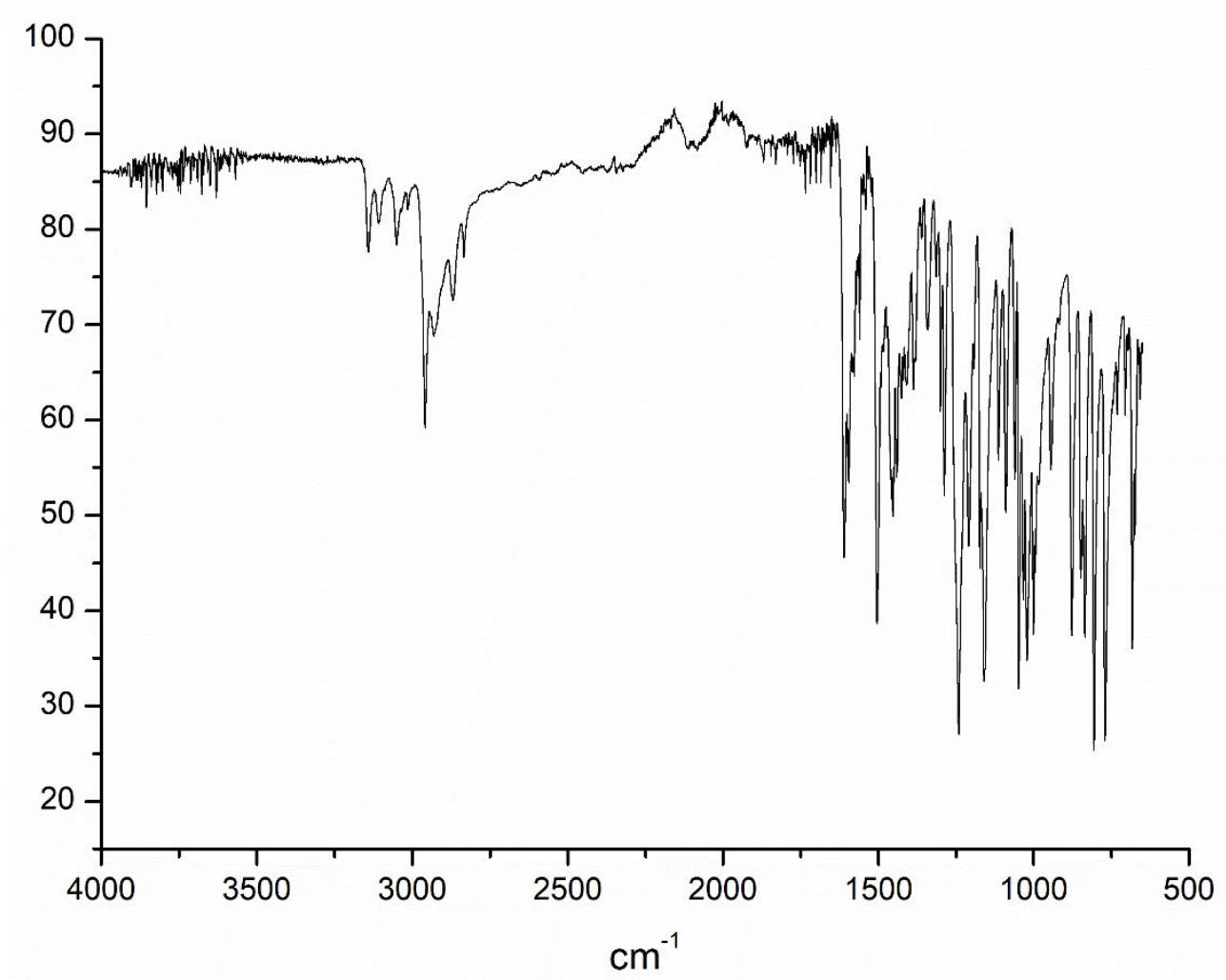

Figure S79. IR spectrum of compound $\mathbf{3 i}$. 


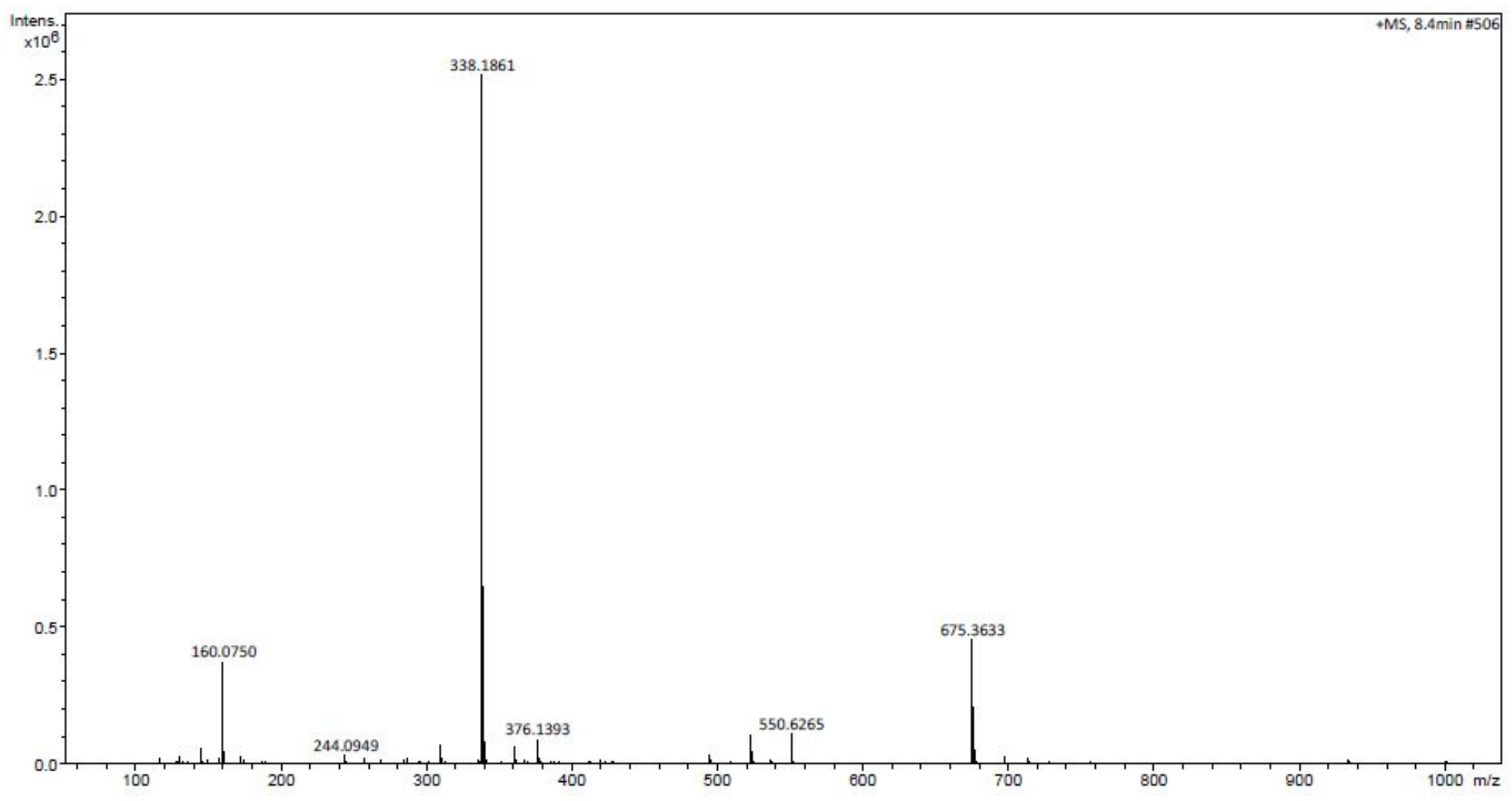

Figure S80. HRMS spectrum of compound $3 \mathbf{i}$. 

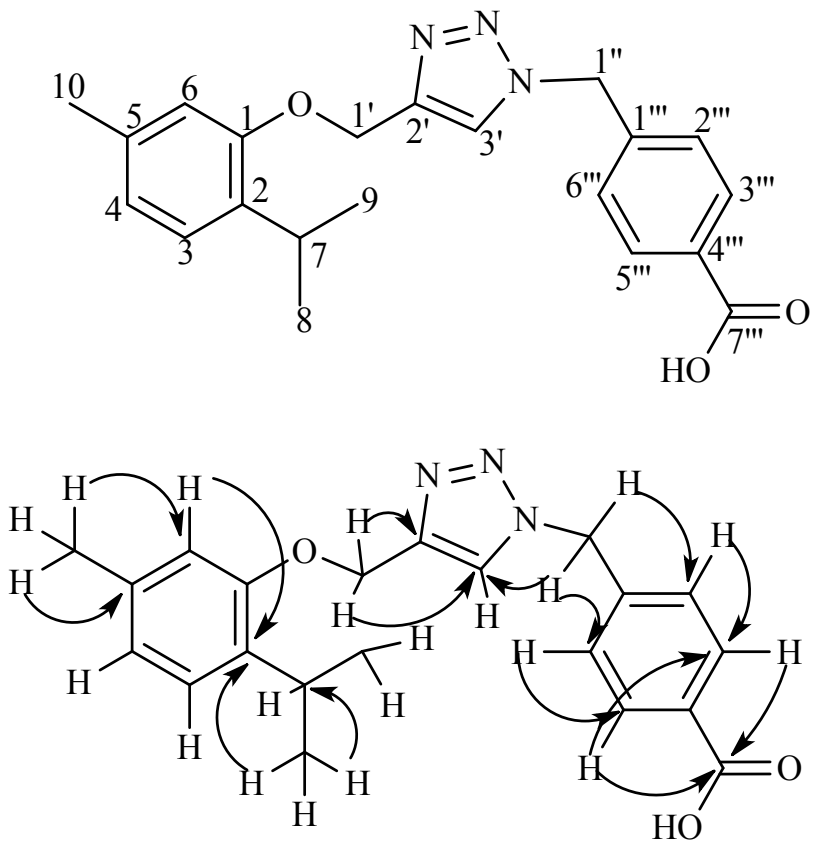

Infra-red

Mass spectrometry

${ }^{1}$ H NMR (400 MHz, $1.05\left(6 \mathrm{H}, \mathrm{d}, \mathrm{CH}_{3}-8\right.$ and $\left.\mathrm{CH}_{3}-9\right) ; 2.23(3 \mathrm{H}, \mathrm{s}$, DMSO- $\left.d_{6}\right)$

$\left.\mathrm{CH}_{3}-10\right) ; 3.06-3.12(1 \mathrm{H}, \mathrm{m}, \mathrm{H}-7) ; 5.10(2 \mathrm{H}, \mathrm{s}$, $\left.\mathrm{CH}_{2}-1{ }^{\prime}\right) ; 5.68\left(2 \mathrm{H}, \mathrm{s}, \mathrm{CH}_{2}-1{ }^{\prime}\right) ; 6.69\left(1 \mathrm{H}, \mathrm{d}, J_{4,3}\right.$ $7.82, \mathrm{H}-4) ; 6.90(1 \mathrm{H}, \mathrm{s}, \mathrm{H}-6) ; 7.01\left(1 \mathrm{H}, \mathrm{d}, J_{3,4}\right.$ 7.82, H-3); $7.32\left(2 \mathrm{H}, \mathrm{d}, J_{2},{ }^{\prime},{ }_{5}, 8.60, \mathrm{H}-2,{ }^{\prime \prime}\right.$, and H-6,', ) 7.89 (2H, d, $J_{3},{ }^{\prime}, 6,, 8.60, \mathrm{H}-3$ ', ', and H5 ', $) ; 8.25$ (1H, s, H-triazole).

${ }^{13}$ C NMR (100 MHz, 21.38 (C-10); 23.06 (C-8, C-9); 26.42 (C-7); DMSO- $d_{6}$ ) 52.81 (C-1'”); $61.98\left(\mathrm{C}-1^{\prime}\right) ; 113.68$ (C-6); 121.88 (C-4); 125.03 (C-3'); 126.06 (C-3); 128.16 (C-2"', and C-6"'); 130.11 (C-3"', and C-5,'); 133.85 (C-2); 136.26 (C-5); 140.86 (C1','); 143.94 (C-2'); 155.43 (C-1); 167.47 (C$\mathrm{OOH})$.

2951 (C-H in $\mathrm{CH}_{2}-1$ ', $\mathrm{CH}_{2-1}$, $\mathrm{CH}-7, \mathrm{CH}_{3}-8$ $\left.\mathrm{CH}_{3}-9, \mathrm{CH}_{3}-10\right) ; 1709$ ( $\mathrm{C}=\mathrm{O}$ in $\mathrm{C}-7{ }^{\prime}$, ') 1254 and 1022 (ether in $\mathrm{CH}_{2}-1$ ' and $\mathrm{C}-1$ ).

HRESIMS [M + H $]^{+}$Found: 366.1817 Calc. for $\mathrm{C}_{21} \mathrm{H}_{24} \mathrm{~N}_{3} \mathrm{O}_{3}{ }^{+}: 366.1818$.

Table S13. Spectroscopic data for compound $\mathbf{3 j}$.

Figure S81. Compound $\mathbf{3 j}$ and main $\mathrm{HMBC}$ correlation $J$ in $\mathrm{Hz}$. 

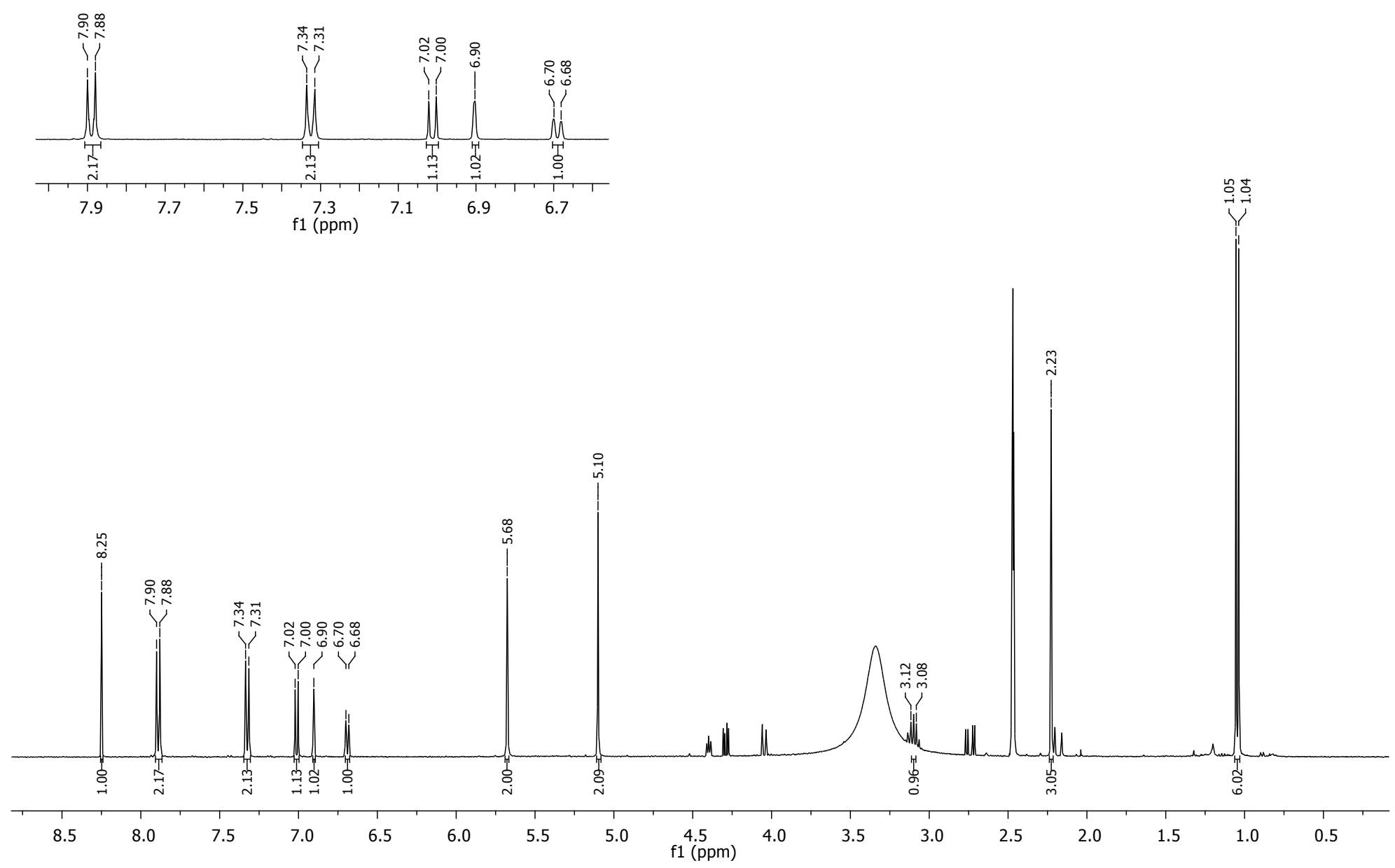

Figure S82. ${ }^{1} \mathrm{H}$ NMR spectrum (400 MHz, DMSO- $\left.d_{6}\right)$ of compound (4-((4-((2-isopropyl-5-methylphenoxy)methyl)-1H-1,2,3-triazol-1-yl)methyl)benzoic acid) $(3 \mathbf{j})$. 


$$
\text { لن. }
$$


Figure S83. ${ }^{13} \mathrm{C}$ NMR spectrum (100 MHz, DMSO- $\left.d_{6}\right)$ of compound (4-((4-((2-isopropyl-5-methylphenoxy)methyl)-1 $H$-1,2,3-triazol-1-yl)methyl)benzoic acid) (3j).

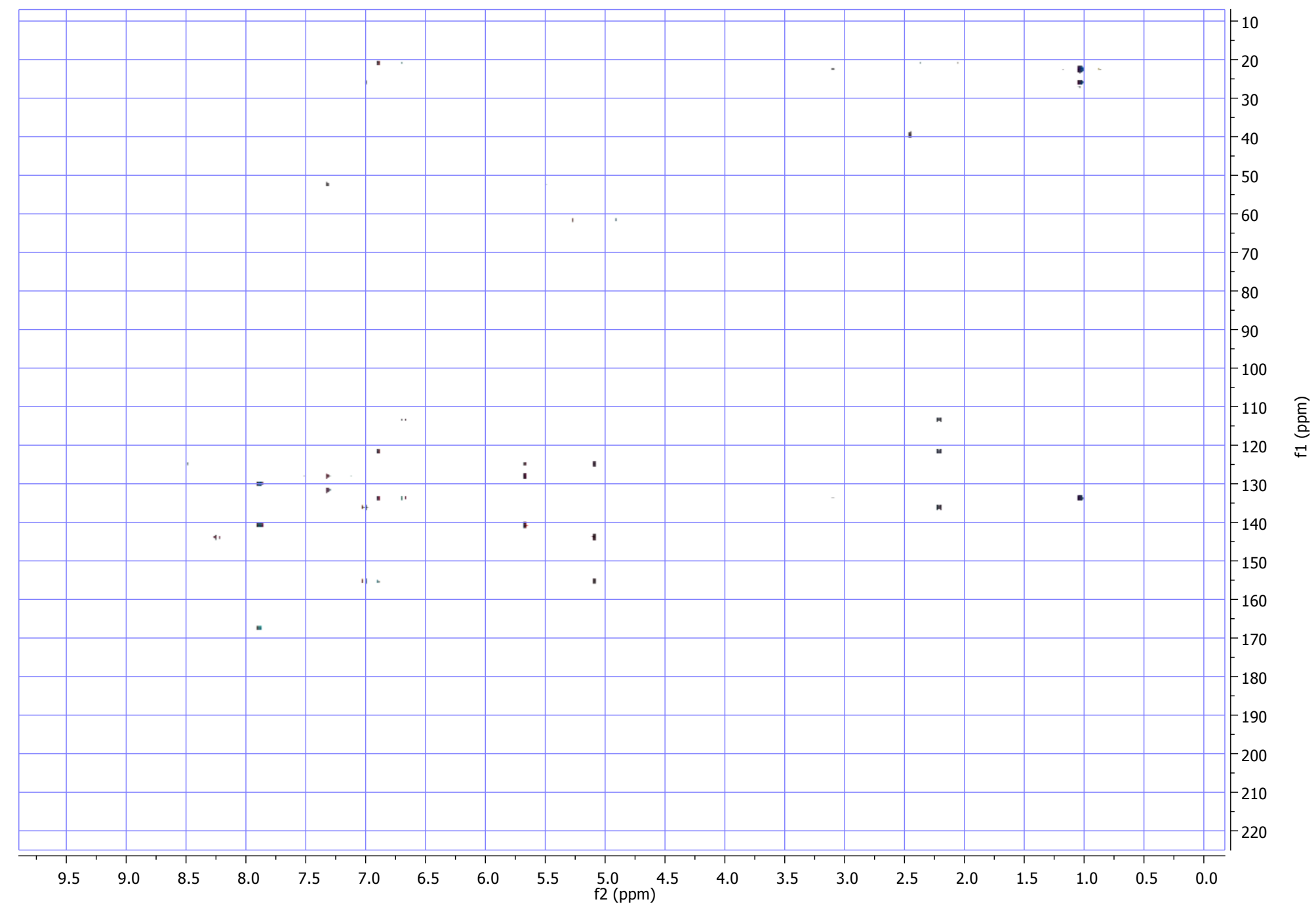


Figure S84 - HMBC spectrum (400 MHz, DMSO- $\left.d_{6}\right)$ of the compound (4-((4-((2-isopropyl-5-methylphenoxy)methyl)-1H-1,2,3-triazol-1-yl)methyl)benzoic acid) (3j).

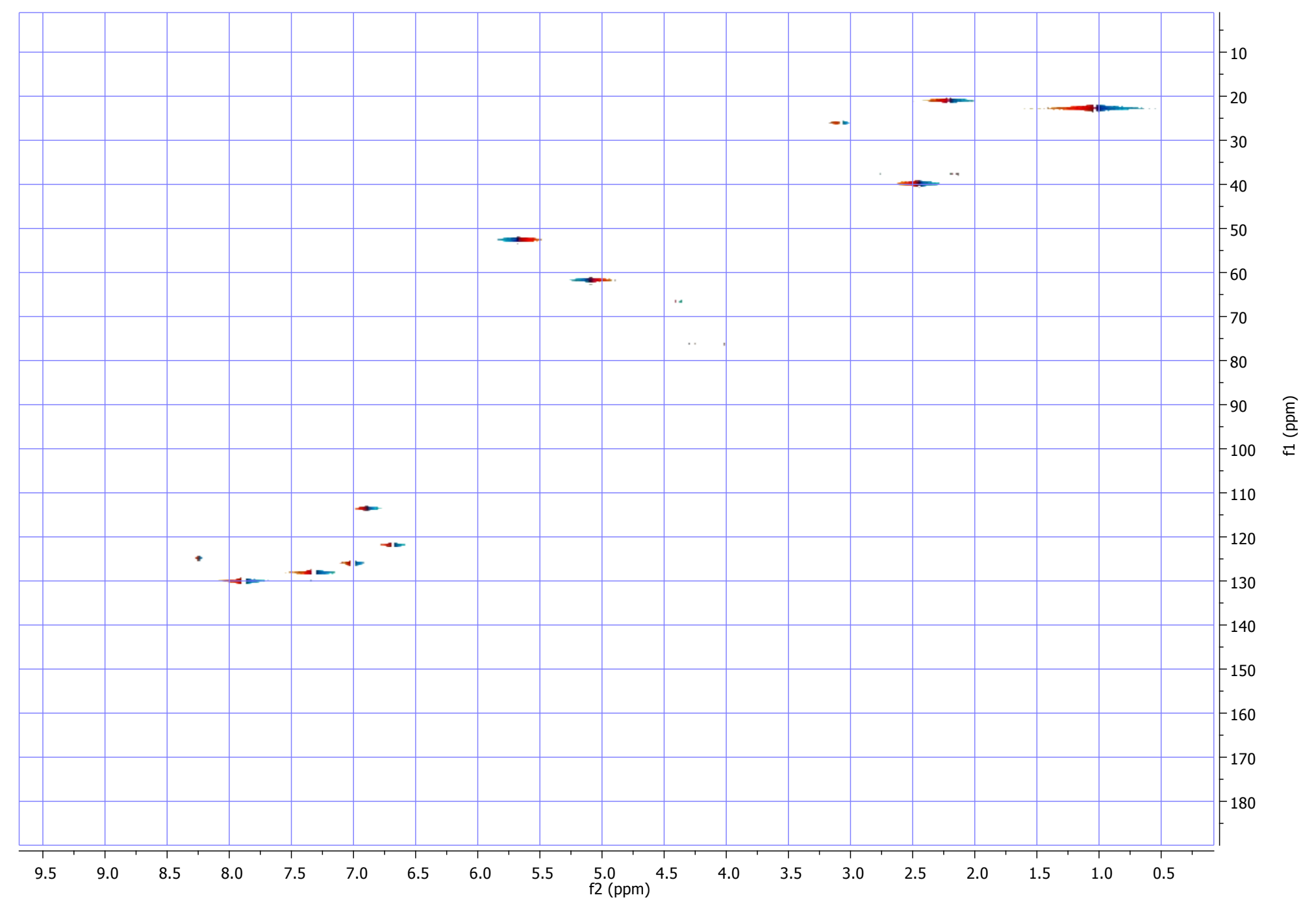


Figure S85 - HSQC spectrum (400 MHz, DMSO- $\left.d_{6}\right)$ of the compound (4-((4-((2-isopropyl-5-methylphenoxy)methyl)-1 $H$-1,2,3-triazol-1-yl)methyl)benzoic acid) $(\mathbf{3 j})$. 


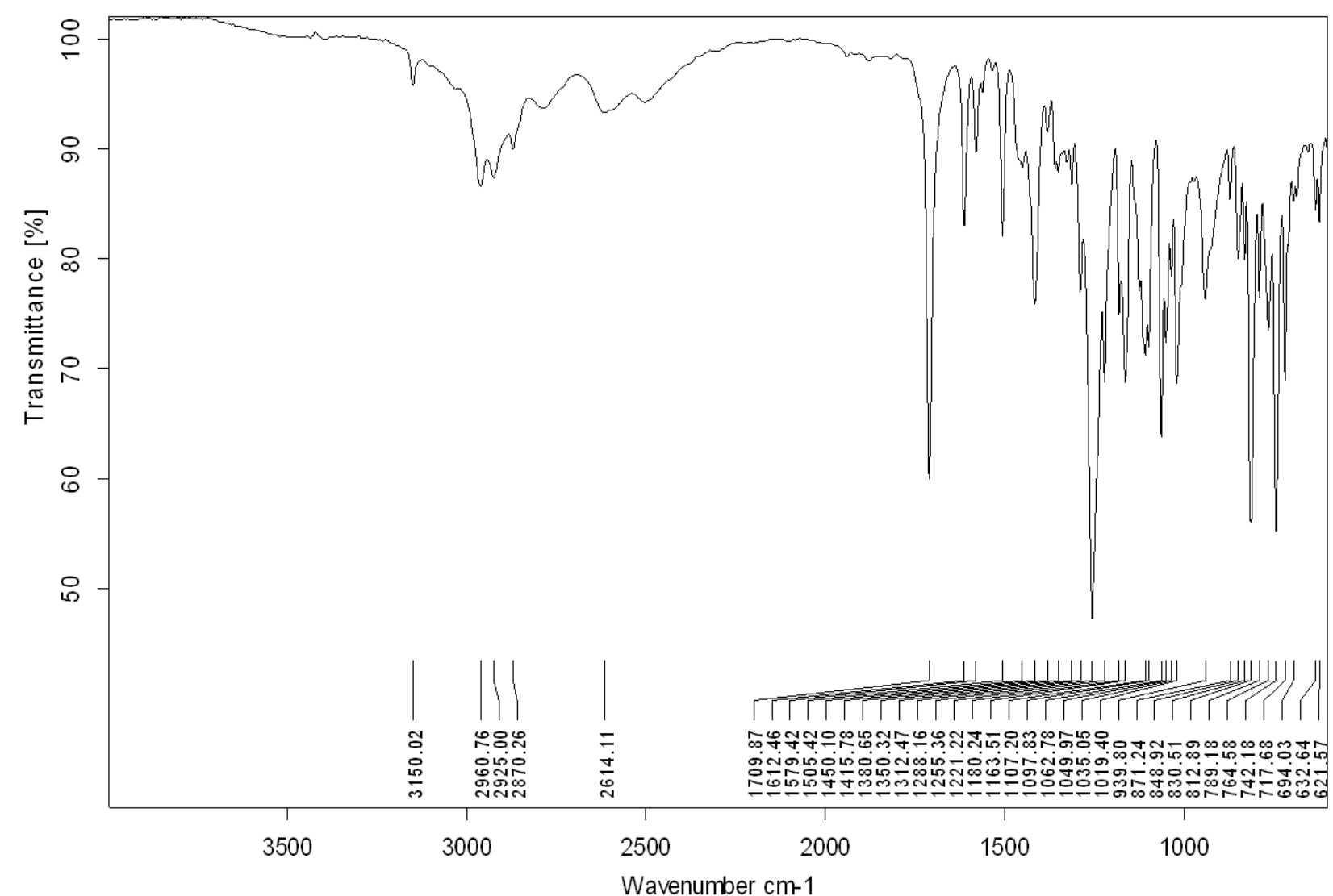

\begin{tabular}{|lll|l|}
\hline C:IPROGRAM FILESIOPUS_65IMEASIPedrol28102020IRayma_41_3J.0 $\quad$ Rayma_41_3J po & $28 / 10 / 2020$ \\
\hline
\end{tabular}

Page 1/1

Figure S86. IR spectrum of compound $\mathbf{3 j}$. 


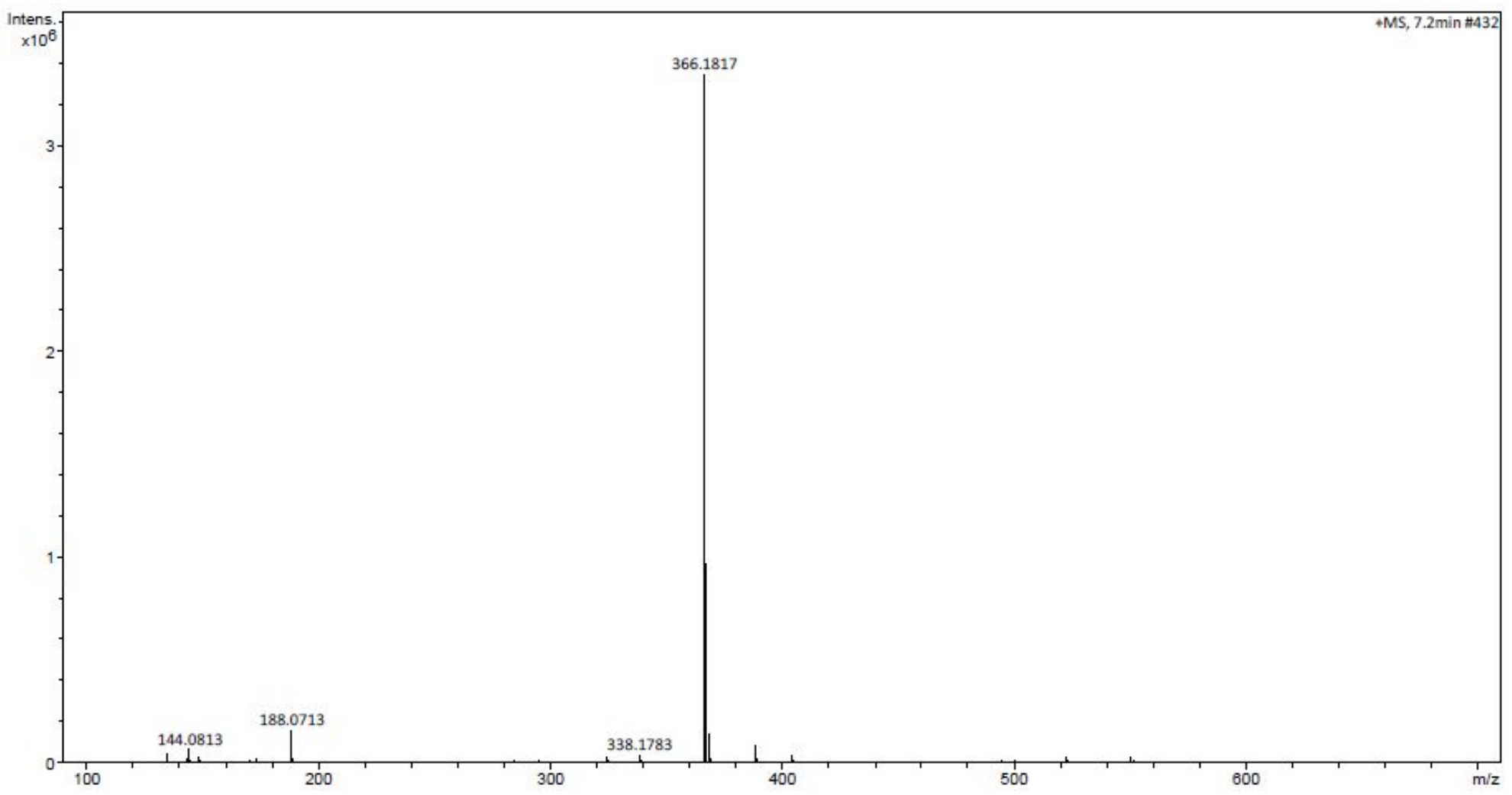

Figure S87. HRMS spectrum of compound $\mathbf{3 j}$. 


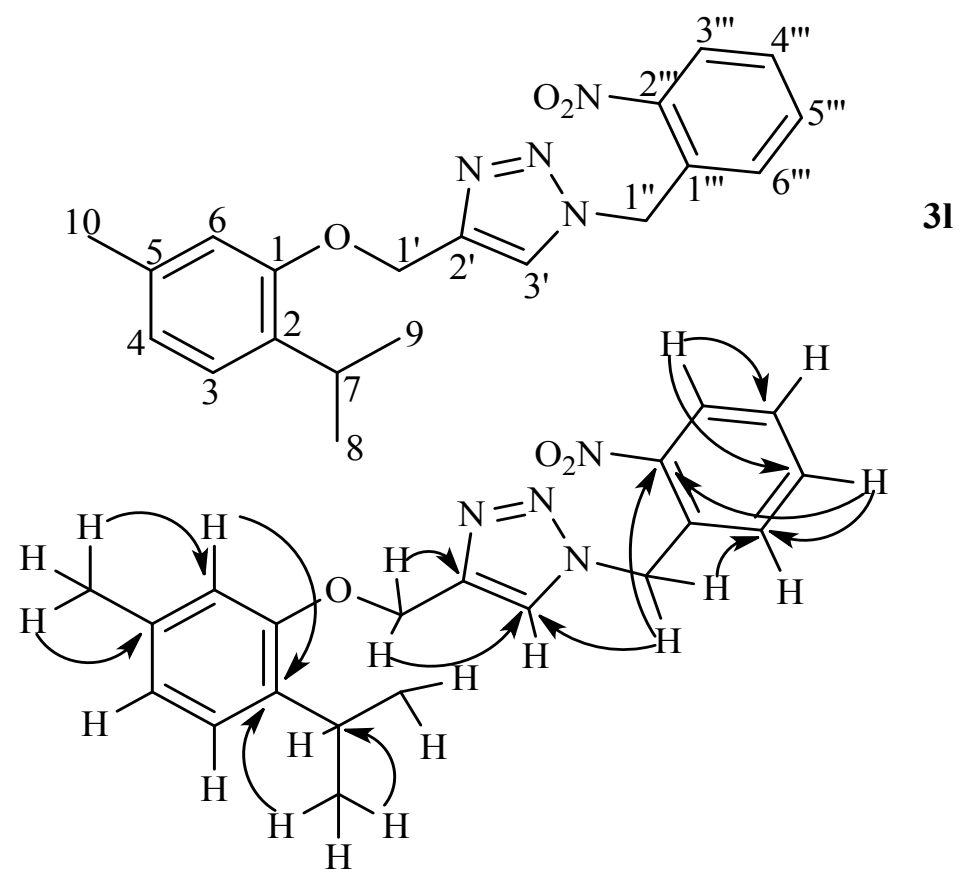

Figure S88. Compound 3k and main HMBC correlation $J$ in $\mathrm{Hz}$.
${ }^{1} \mathbf{H}$ NMR (400 MHz, $1.06\left(6 \mathrm{H}, \mathrm{d}, \mathrm{CH}_{3}-8\right.$ and $\left.\mathrm{CH}_{3}-9\right)$; $2.23\left(3 \mathrm{H}, \mathrm{s}, \mathrm{CH}_{3}\right.$ DMSO- $\left.d_{6}\right)$

$10) ; 3.08-3.15(1 \mathrm{H}, \mathrm{m}, \mathrm{H}-7) ; 5.14\left(2 \mathrm{H}, \mathrm{s}, \mathrm{CH}_{2-}\right.$ 1 '); $5.96\left(2 \mathrm{H}, \mathrm{s}, \mathrm{CH}_{2}-1\right.$ ''); $6.69\left(1 \mathrm{H}, \mathrm{d}, J_{4,3} 7.82\right.$ H-4); 6.91 (1H, s, H-6); 6.99 (1H, d, $J_{6, ",},{ }^{,}, 8.21$, H-6', $) ; 7.01\left(1 \mathrm{H}, \mathrm{d}, J_{3,4} 7.82, \mathrm{H}-3\right) ; 7.60(1 \mathrm{H}, \mathrm{t}$, $\left.J_{5},,{ }^{\prime},, 8.21, \mathrm{H}-5,{ }^{\prime}, '\right) ; 7.69\left(1 \mathrm{H}, \mathrm{t}, J_{4},,{ }^{\prime},{ }^{\prime}, 8.21, \mathrm{H}-\right.$

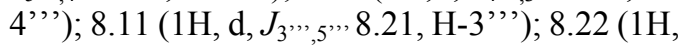
s, H-triazole).

${ }^{13}$ C NMR (100 MHz, 21.36 (C-10); 23.03 (C-8, C-9); 26.49 (C-7) DMSO- $\left.d_{6}\right)$ 50.38 (C-1'’); $61.96\left(\mathrm{C}-1^{\prime}\right) ; 113.65$ (C-6); 121.88 (C-4); 125.48 (C-3'); 125.52 (C-3'’'); 126.06 (C-3); 130.03 (C-5','); 130.29 (C-6",'); 131.40 (C-1',') 133.83 (C-2); 134.71 (C-4'"'); 136.27 (C-5); 143.98 (C-2'); 147.96 (C-2'’'); $155.42(\mathrm{C}-1)$.

Infra-red $\left(\mathrm{cm}^{-1}\right)$

2919 (C-H in $\mathrm{CH}_{2}-1$ ', $\mathrm{CH}-7, \mathrm{CH}_{3}-8, \mathrm{CH}_{3}-9$, $\left.\mathrm{CH}_{3}-10\right) ; 1516$ and $1345\left(\mathrm{NO}_{2}\right.$ in $\left.\mathrm{C}-2{ }^{\prime},{ }^{\prime}\right) ; 1247$ and 1040 (ether in $\mathrm{CH}_{2}-1$ ' and $\mathrm{C}-1$ ).

Mass spectrometry HRESIMS $[\mathrm{M}+\mathrm{H}]^{+}$Found: 367.1693 Calc. for $\mathrm{C}_{20} \mathrm{H}_{23} \mathrm{~N}_{4} \mathrm{O}_{3}{ }^{+}: 367.1770$.

Table S14. Spectroscopic data for compound $\mathbf{3 k}$. 

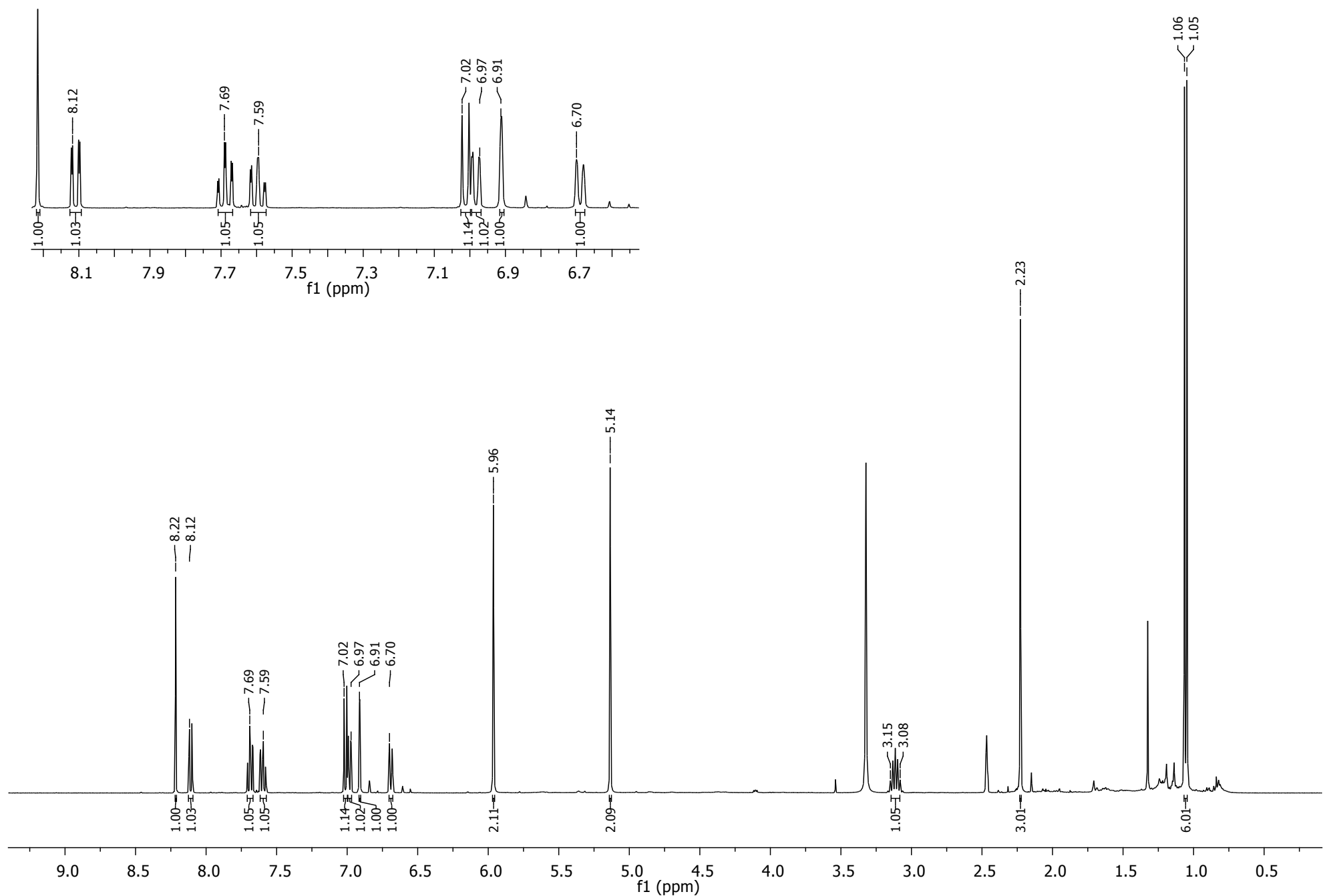

Figure S89. ${ }^{1} \mathrm{H}$ NMR spectrum (400 MHz, DMSO- $d_{6}$ ) of compound (4-((2-isopropyl-5-methylphenoxy)methyl)-1-(2-nitrobenzyl)-1 $H$-1,2,3-triazole) (3k). 


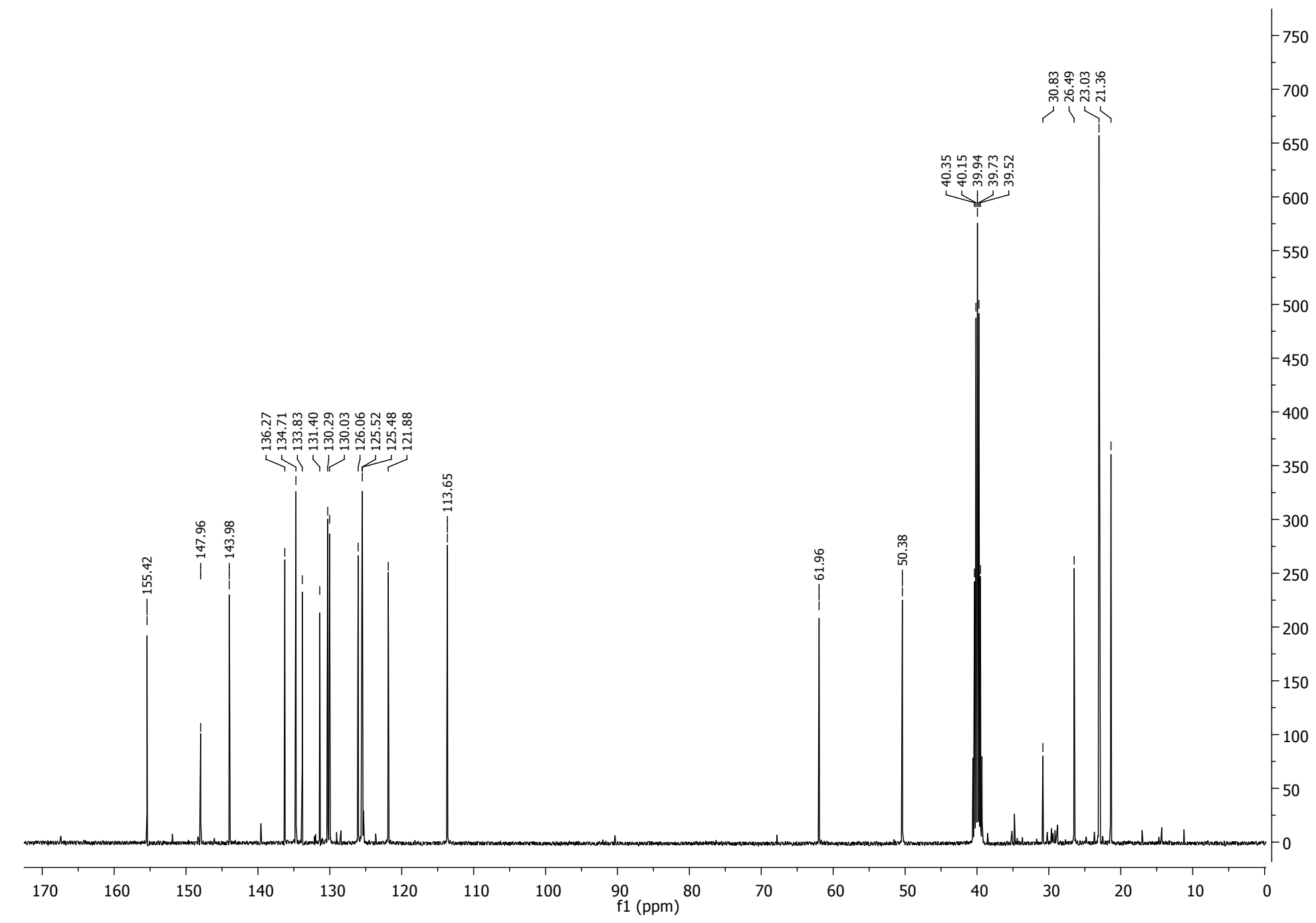

Figure S90. ${ }^{13} \mathrm{C}$ NMR spectrum (100 MHz, DMSO- $\left.d_{6}\right)$ of compound (4-((2-isopropyl-5-methylphenoxy)methyl)-1-(2-nitrobenzyl)-1H-1,2,3-triazole) (3k). 


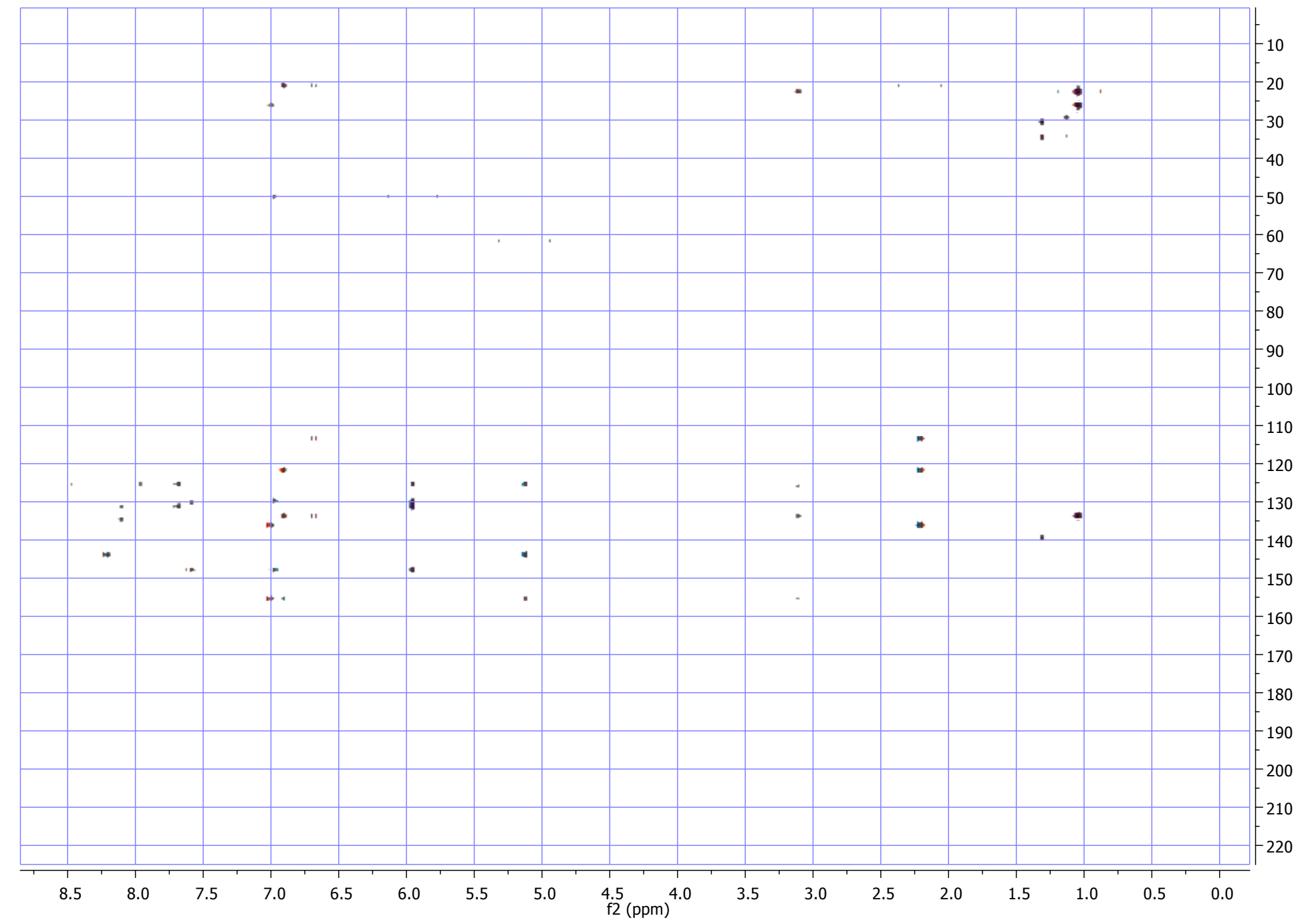

Figure S91 - HMBC spectrum (400 MHz, DMSO- $\left.d_{6}\right)$ of the compound (4-((2-isopropyl-5-methylphenoxy)methyl)-1-(2-nitrobenzyl)-1 H-1,2,3-triazole) (3k). 


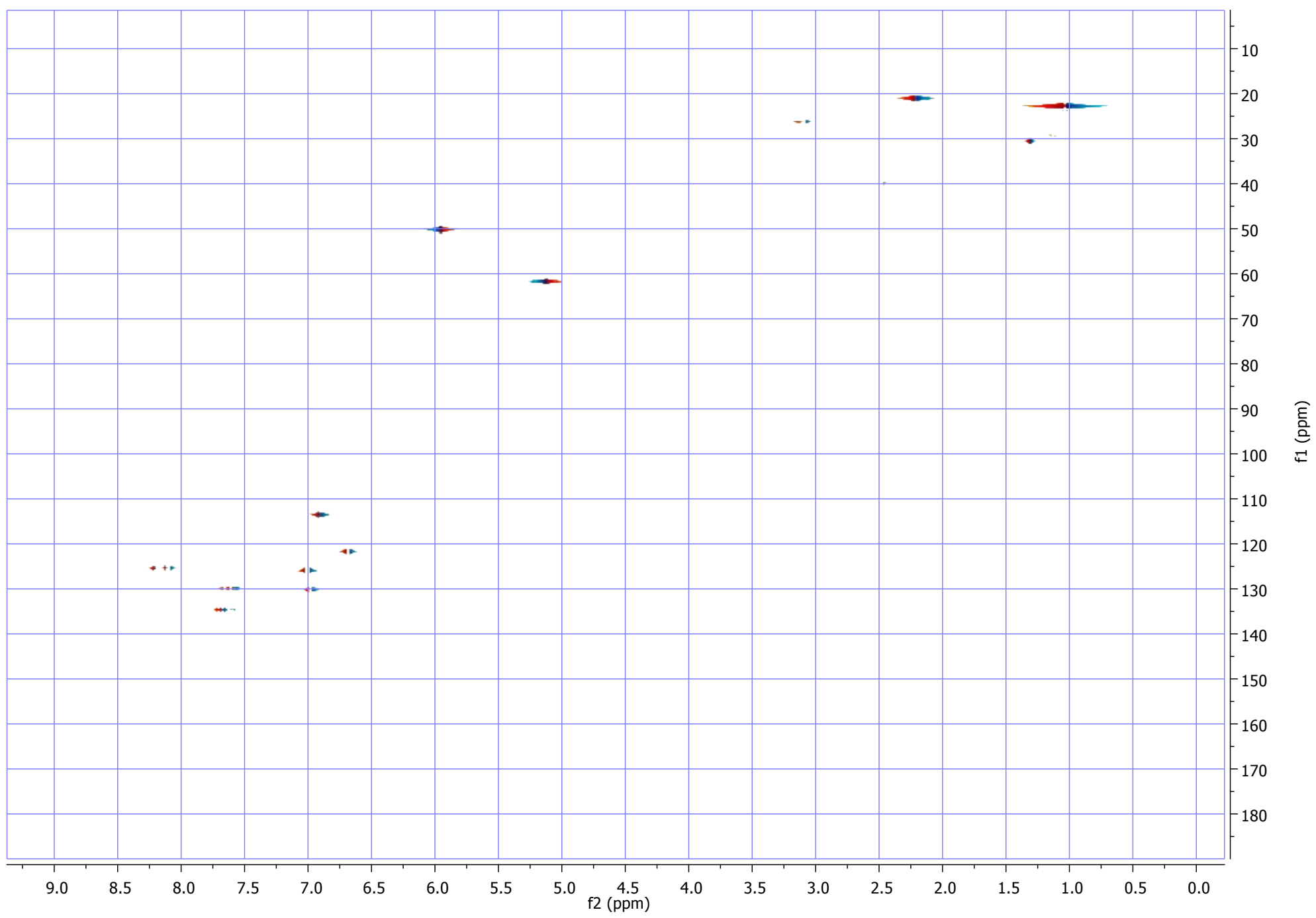

Figure S92 - HSQC spectrum (400 MHz, DMSO- $d_{6}$ ) of the compound (4-((2-isopropyl-5-methylphenoxy)methyl)-1-(2-nitrobenzyl)-1H-1,2,3-triazole) (3k). 


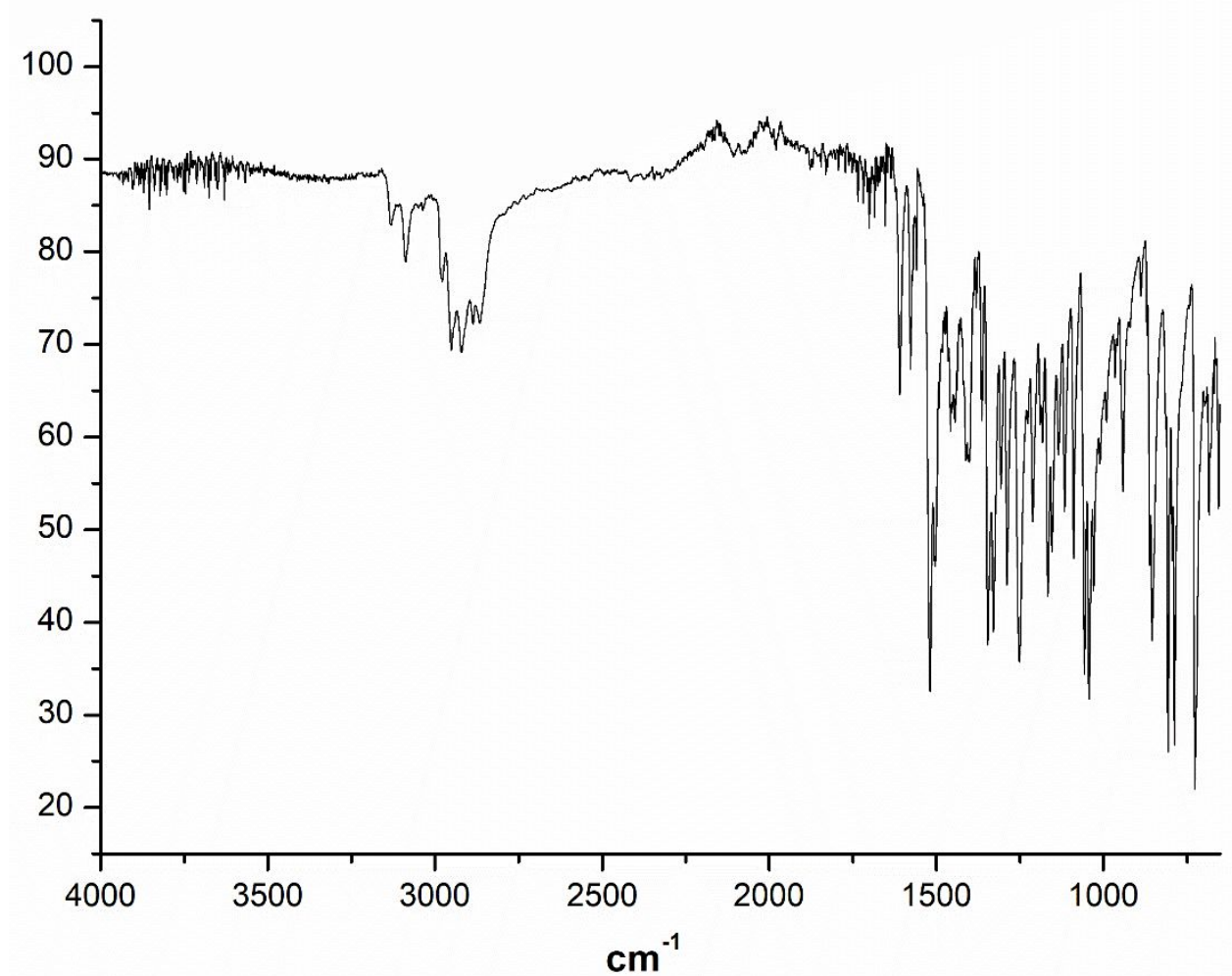

Figure S93. IR spectrum of compound 3k. 


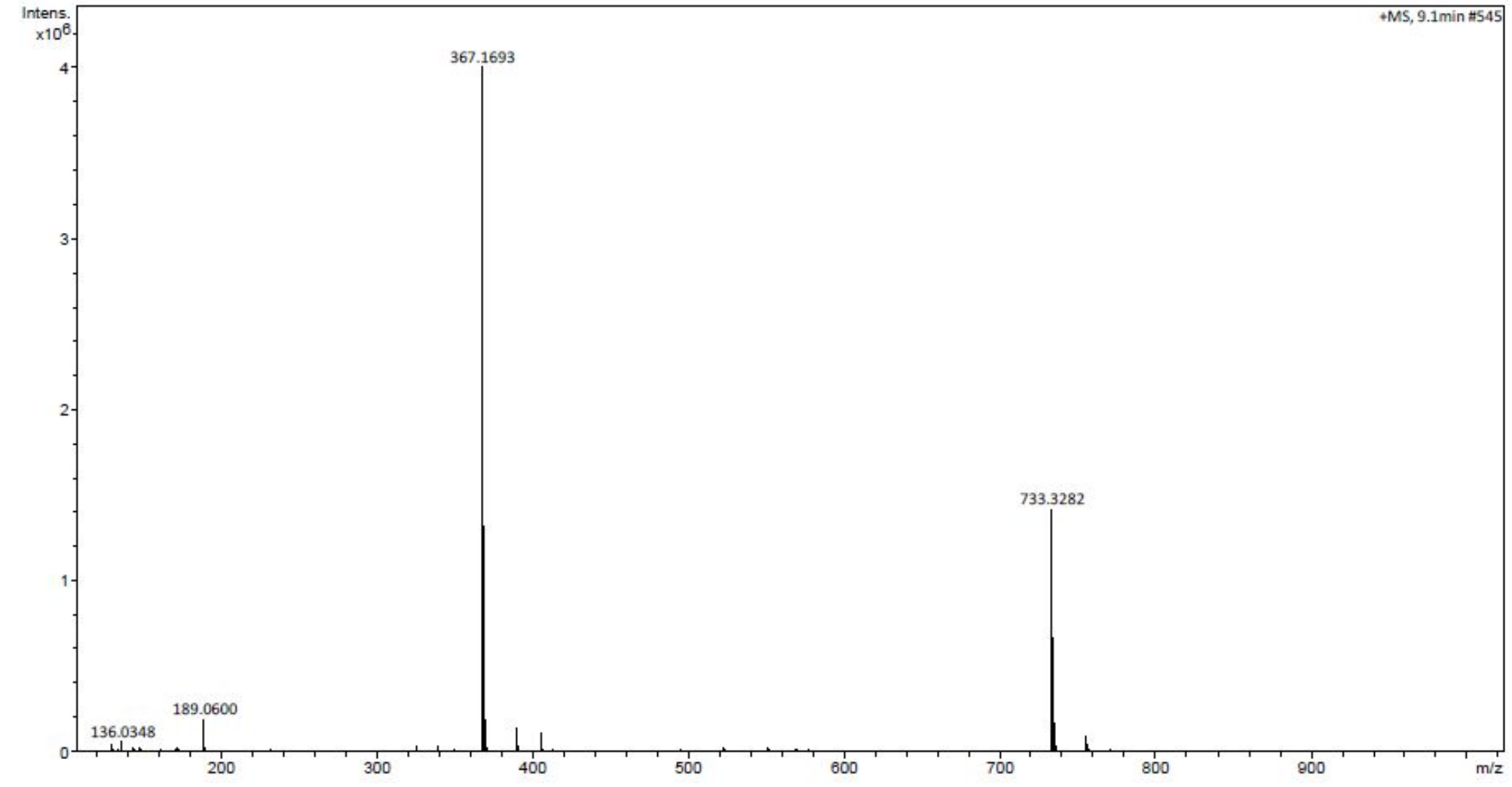

Figure S94. HRMS spectrum of compound 3k. 


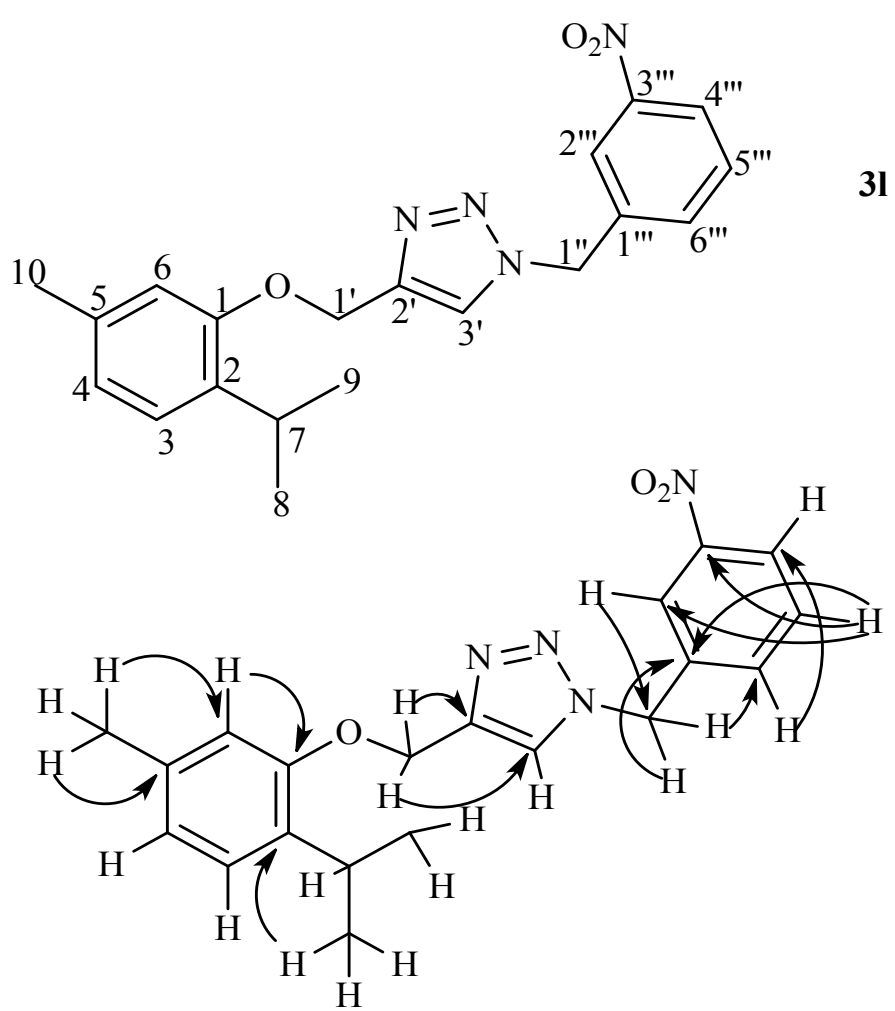

Figure S95. Compound $\mathbf{3 1}$ and main $\mathrm{HMBC}$ correlation $J$ in $\mathrm{Hz}$.
${ }^{1} \mathrm{H}$ NMR (400 MHz, $1.02\left(6 \mathrm{H}, \mathrm{d}, \mathrm{CH}_{3}-8\right.$ and $\left.\mathrm{CH}_{3}-9\right) ; 2.21(3 \mathrm{H}, \mathrm{s}$, DMSO- $\left.d_{6}\right)$

$\left.\mathrm{CH}_{3}-10\right) ; 3.04-3.11(1 \mathrm{H}, \mathrm{m}, \mathrm{H}-7) ; 5.09(2 \mathrm{H}, \mathrm{s}$, $\mathrm{CH}_{2}-1$ '); $5.75\left(1 \mathrm{H}, \mathrm{s}, \mathrm{CH}_{2}-1\right.$ '’); $6.68\left(1 \mathrm{H}, \mathrm{d}, J_{4,3}\right.$ $7.82, \mathrm{H}-4) ; 6.80(1 \mathrm{H}, \mathrm{s}, \mathrm{H}-6) ; 6.99\left(1 \mathrm{H}, \mathrm{d}, J_{3,4}\right.$ 7.82, H-3); 7.64 (1H, t, $\left.J_{5},,{ }_{2},{ }^{\prime}, 8.60, \mathrm{H}-5{ }^{\prime},{ }^{\prime}\right) ; 7.73$ (1H, d, $\left.J_{6}, ", 5,, 8.60, \mathrm{H}-6,, '\right) ; 8.15\left(2 \mathrm{H}, \mathrm{m}, J_{2},{ }^{\prime}, 5^{\prime}\right.$, $8.60 ; J_{4},{ }^{\prime},{ }^{\prime}, 8.60, \mathrm{H}-4, \cdot$, and $\left.\mathrm{H}-2{ }^{\prime \prime \prime}\right) ; 8.29(1 \mathrm{H}$, s, H-triazole).

${ }^{13}$ C NMR (100 MHz, 21.30 (C-10); 22.99 (C-8, C-9); 26.41 (C-7); DMSO- $d_{6}$ ) 52.21 (C-1'); 61.89 (C-1'); 113.66 (C-6); 121.95 (C-4); 123.05 (C-2','); 123.54 (C-4'”'); 125.07 (C-3); 130.83 (C-5','); 133.87 (C-2); 135.06 (C-6"'); 136.33 (C-5); 138.48 (C-1'”); 144.10 (C-2'), 148.28 (C-3'’'); 155.37 (C-1).

Infra-red $\left(\mathrm{cm}^{-1}\right)$ 2951 (C-H in $\mathrm{CH}_{2}-1$ ', $\mathrm{CH}_{2}-1$ ', $\mathrm{CH}-7, \mathrm{CH}_{3}-8$, $\left.\mathrm{CH}_{3}-9, \mathrm{CH}_{3}-10\right)$; 1522 and $1336\left(\mathrm{NO}_{2}\right.$ in $\mathrm{C}-3{ }^{\prime \prime}$ '); 1254 and 1022 (ether in $\mathrm{CH}_{2}-1$ ' and $\mathrm{C}-1$ ).

Mass spectrometry HRESIMS $[\mathrm{M}+\mathrm{H}]^{+}$Found: 367.1764 Calc. for $\mathrm{C}_{20} \mathrm{H}_{23} \mathrm{~N}_{4} \mathrm{O}_{3}{ }^{+}: 367.1770$.

Table S15. Spectroscopic data for compound $\mathbf{3 1 .}$ 

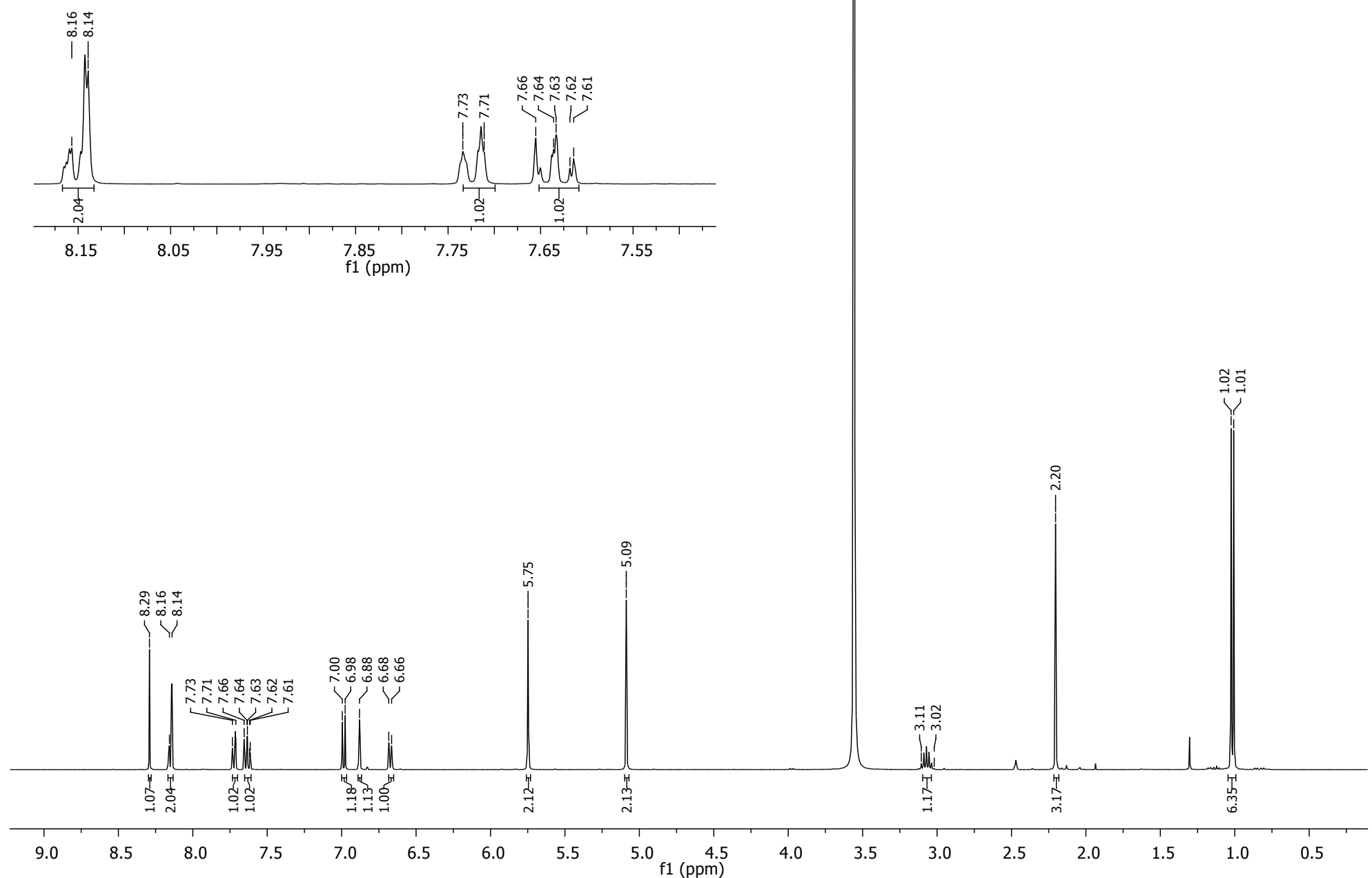

Figure S96. ${ }^{1} \mathrm{H}$ NMR spectrum (400 MHz, DMSO- $d_{6}$ ) of compound (4-((2-isopropyl-5-methylphenoxy)methyl)-1-(3-nitrobenzyl)-1 $H$-1,2,3-triazole) (31). 


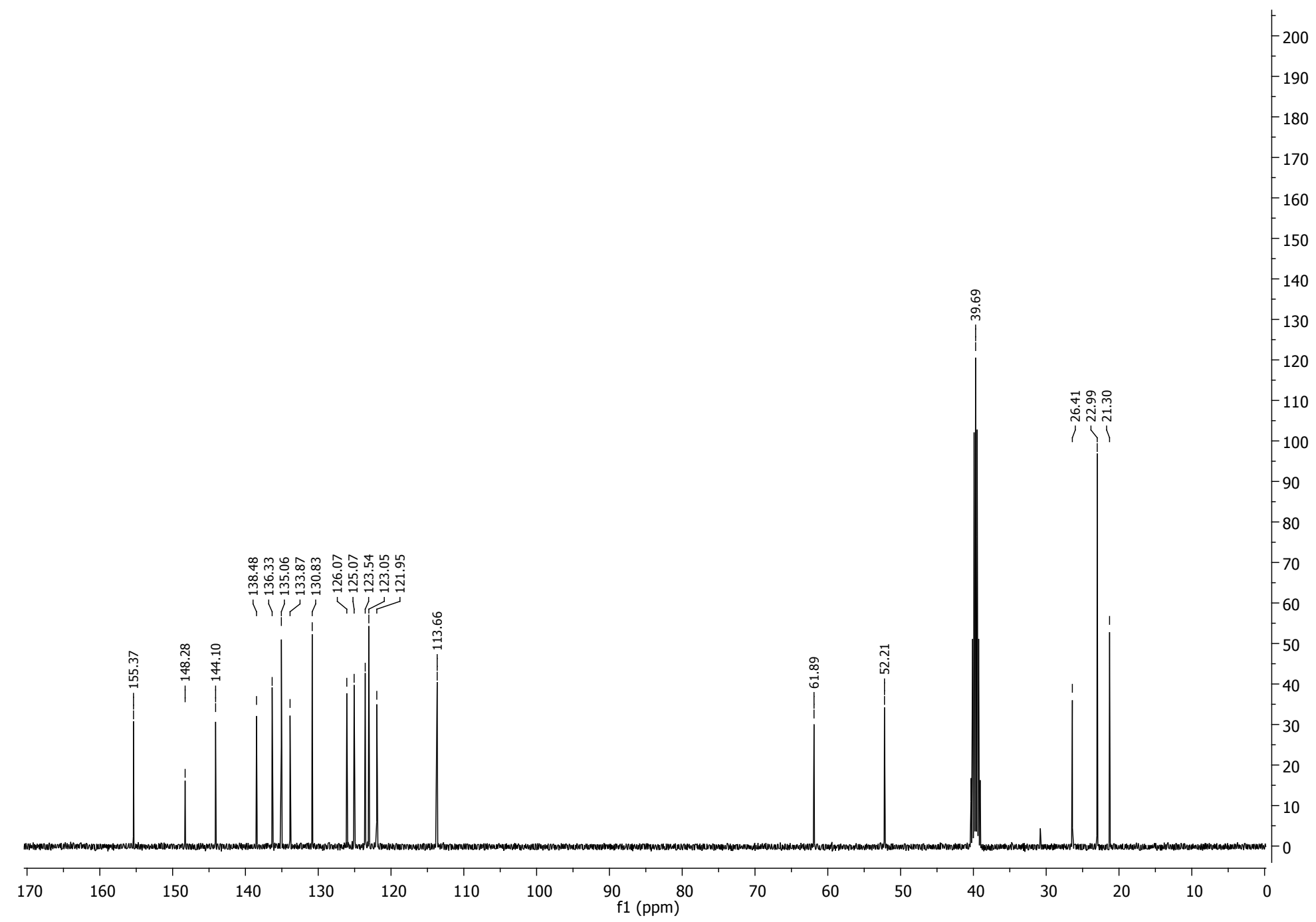

Figure S97. ${ }^{13} \mathrm{C}$ NMR spectrum (100 MHz, DMSO- $\left.d_{6}\right)$ of compound (4-((2-isopropyl-5-methylphenoxy)methyl)-1-(3-nitrobenzyl)-1H-1,2,3-triazole) (31). 


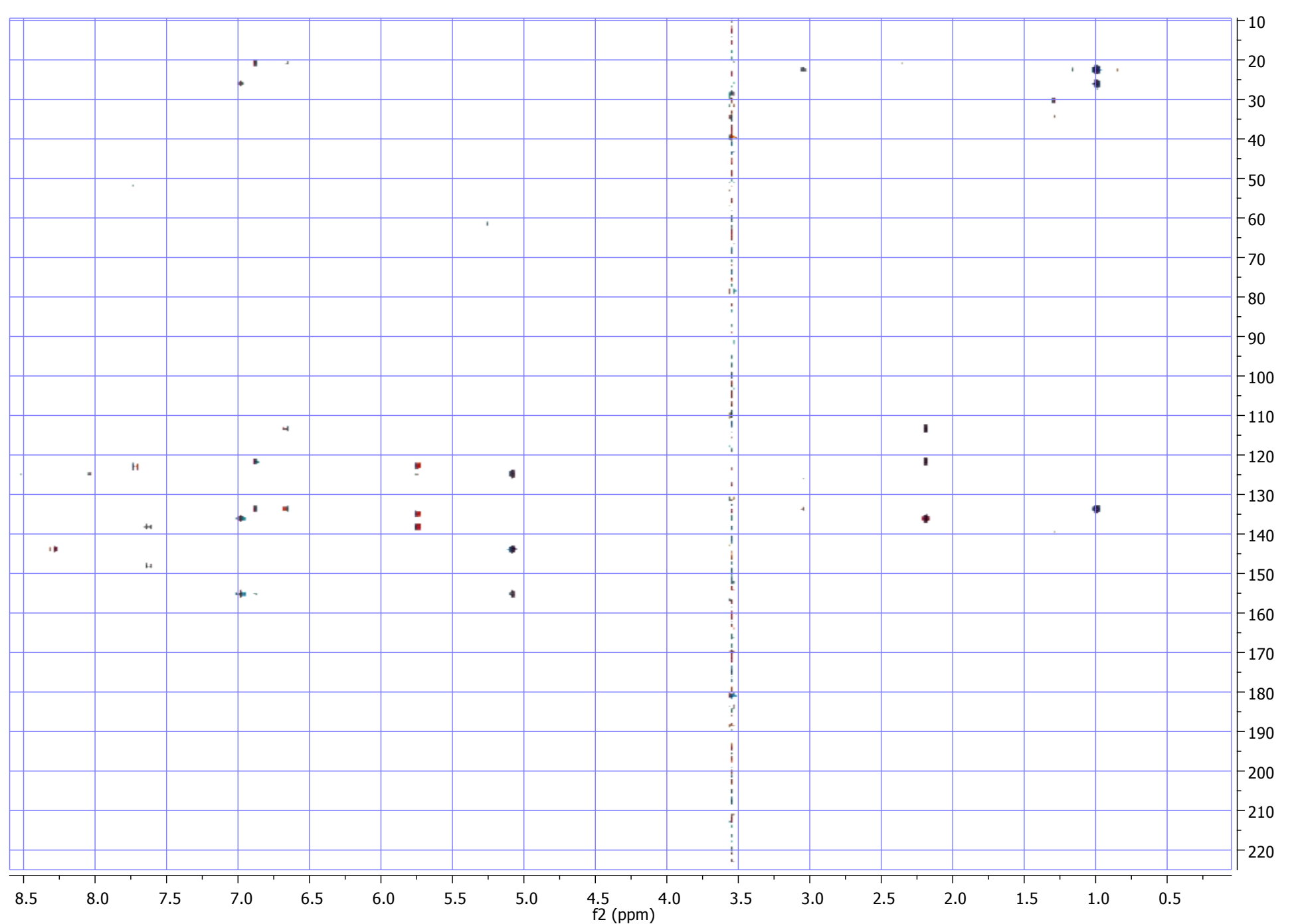

Figure S98 - HMBC spectrum (400 MHz, DMSO- $\left.d_{6}\right)$ of the compound (4-((2-isopropyl-5-methylphenoxy)methyl)-1-(3-nitrobenzyl)-1 H-1,2,3-triazole) (31). 


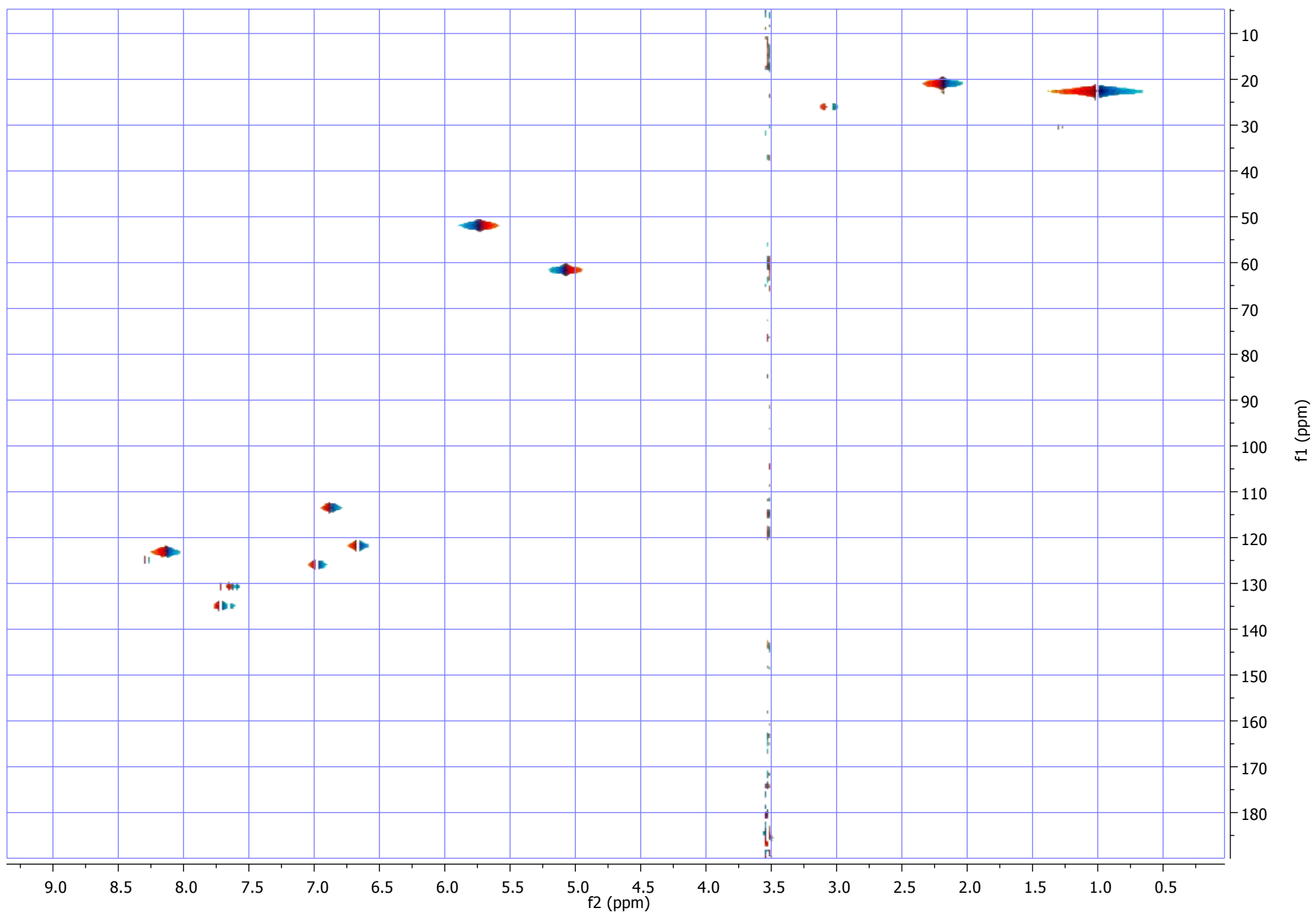

Figure S99 - HSQC spectrum (400 MHz, DMSO- $d_{6}$ ) of the compound (4-((2-isopropyl-5-methylphenoxy)methyl)-1-(3-nitrobenzyl)-1H-1,2,3-triazole) (31). 


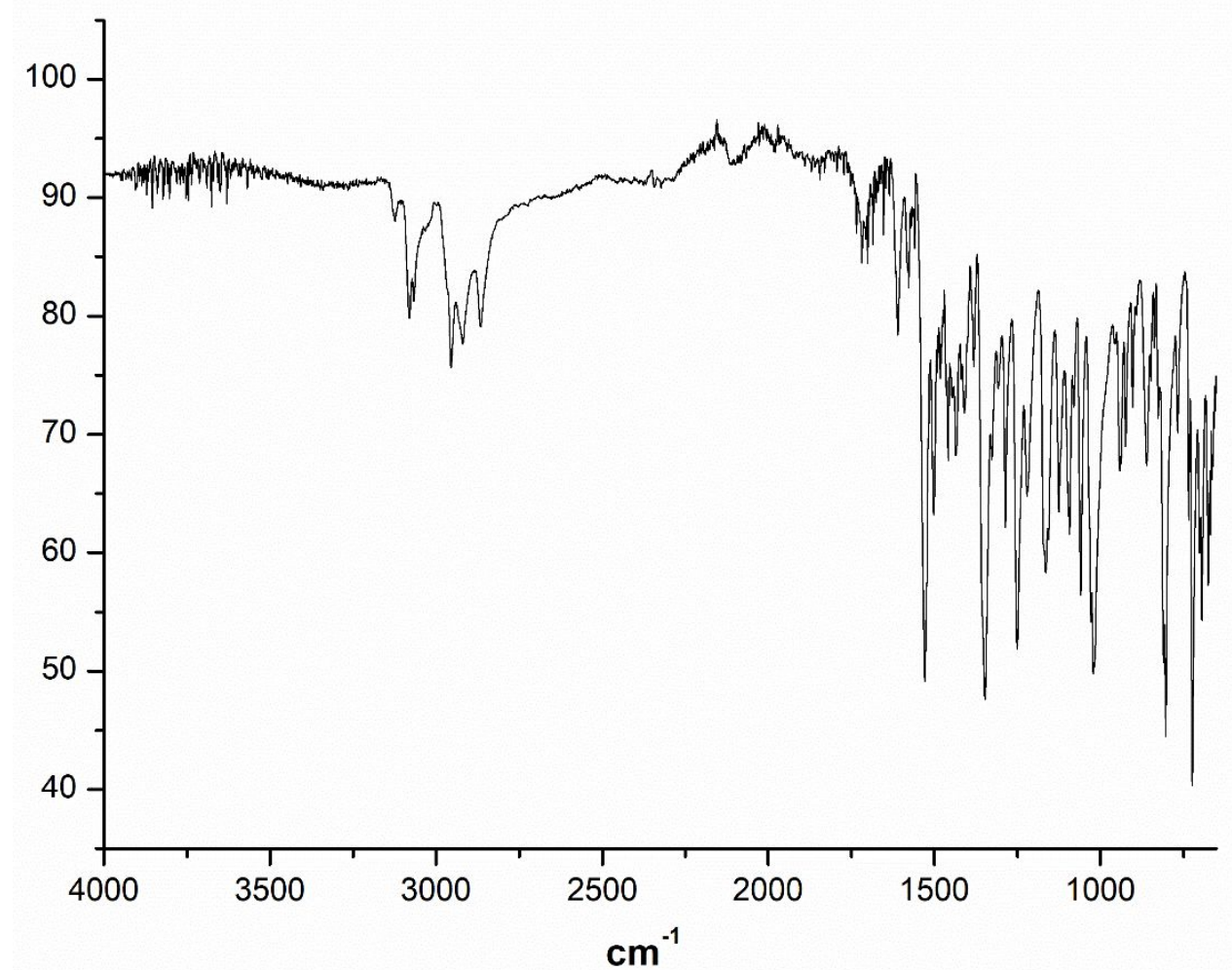

Figure S100. IR spectrum of compound 31. 


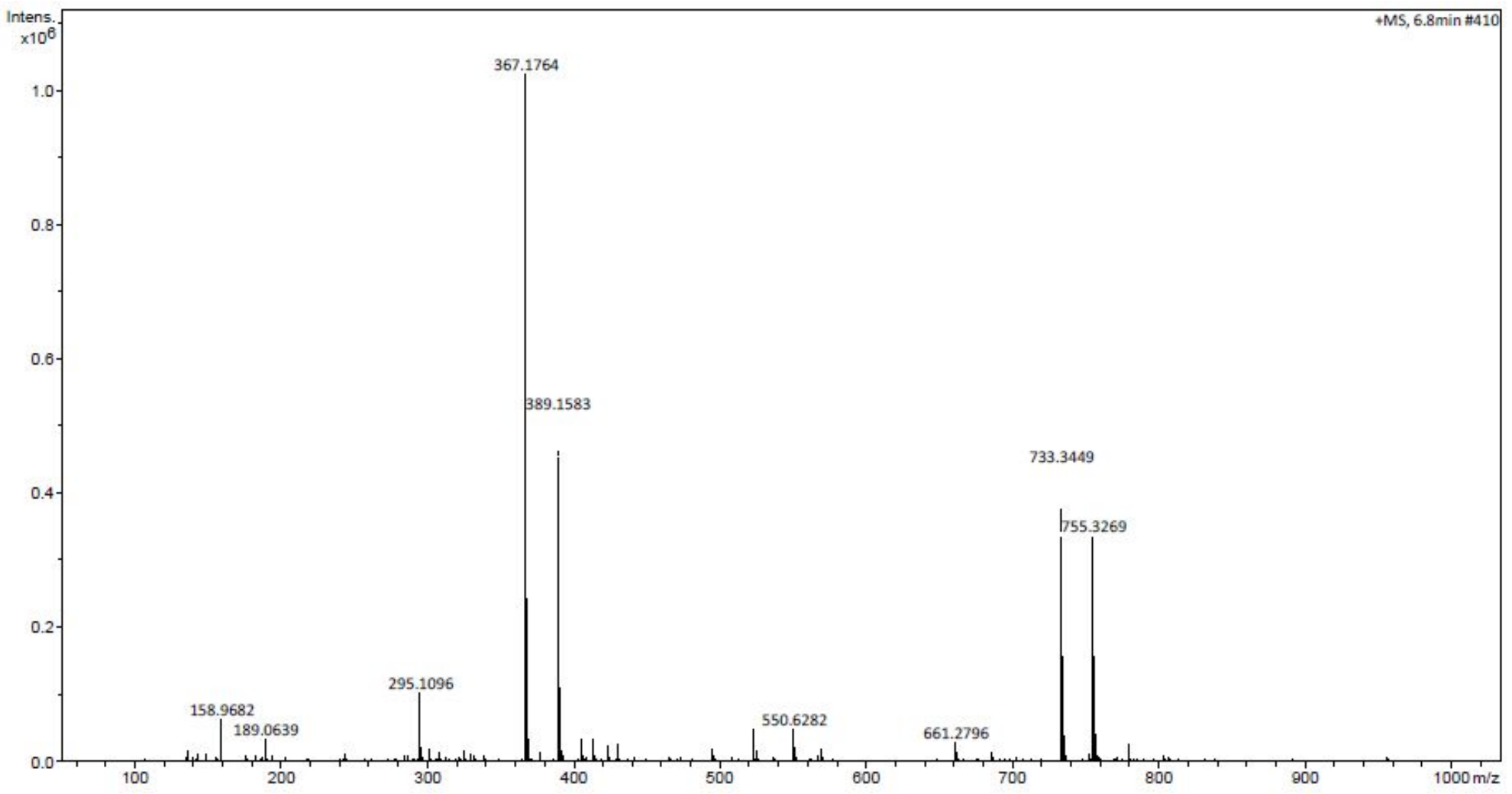

Figure S101. HRMS spectrum of compound 31 . 